\author{
Universidade de São Paulo \\ Instituto de Física \\ Departamento de Física-Matemática
}

\title{
Halos de matéria escura e campos escalares
}

\section{Rafael Ribeiro Brandão}

Orientador: Prof. Dr. Luís Raul Weber Abramo

Trabalho apresentado ao Instituto de Física da Universidade de São Paulo para obtenção do título de Mestre em Ciências.

Banca examinadora:

Prof. Dr. Luís Raul Weber Abramo (IF-USP)

Prof. Dr. Élcio Abdalla (IF-USP)

Prof. Dr. Hugo Vicente Capelato (INPE)

São Paulo 
Para ser grande, sê inteiro: nada

Teu exagera ou exclui.

Sê todo em cada coisa. Põe quanto és

No mínimo que fazes.

Assim em cada lago a lua toda

Brilha, porque alta vive.

Ricardo Reis, Odes 


\section{Agradecimentos}

Esta é muito provavelmente a parte mais esperada e mais prazerosa de ser escrita dentro do processo de elaboração da dissertação. Quem já acompanhou de perto um processo desse tipo sabe das dificuldades que aparecem e provavelmente será capaz de entender o porquê dessa gigantesca vontade de incluir muitas pessoas aqui nessas linhas.

Para começar, agradeço à divindade máxima do meu credo, pela iluminação de um caminho que nem sempre pareceu tão claro quanto eu gostaria. Deixo meus cumprimentos de gratidão também ao Prof. Dr. Raul Abramo, pela paciência e boa-vontade com que me orientou, conduziu o problema me ensinando tantas coisas e tornou possível a realização dessa obra. Agradeço ainda aos meus familiares que, de uma forma ou de outra, foram capazes de dar suporte emocional para que o trabalho fosse concretizado. Não menos importante, um agradecimento ao CNPq pelo apoio financeiro, certamente indispensável.

Um "muito obrigado" especial vai para o pessoal do grupo cósmico: Vinícius Fernandes (Vini), Thiago Pereira, Ronaldo Batista (Gaúcho) e Ivan Yasuda, pelo convívio divertidíssimo e pelas inúmeras discussões frutíferas na lousa da nossa sala, cujos desdobramentos certamente fazem parte desse texto. Muitas palmas vão aqui também para João Assirati, grande guru intelectual no que diz respeito ao universo da física e dos computadores (e provavelmente de vários outros tópicos). Outros que certamente têm vez aqui são Carlos Molina e Mário Baldiotti, antigos vizinhos de sala e que invariavelmente apareciam nas discussões da 321 para dar um xeque-mate no problema. Agradeço ainda ao Rodrigo Fresneda por me ensinar não apenas um muito de teoria de campos mas também que os $14 \mathrm{~km}$ que separam a USP de minha casa podem sim ser vencidos de bicicleta. Mando ainda um alô especial para as moças da secretaria da Física-Matemática: Simone, Amélia e Beth que quebraram muitos e muitos galhos.

O espaço é curto, mas a ausência de nomes não vai ofuscar o brilho de tantos merecedores de agradecimento por uma razão não menos grandiosa que as anteriores: uma incomensurável amizade. Entram aqui o pessoal dos Tákions, vários amigos e amigas incríveis desde os tempos de graduação. Também fazem parte desse time o time de futsal e toda a turma aqui da Física que curtiu bater uma bola no Cepê nas horas de folga, entre uma integral e outra. Leandro Krug, vulgo "Cabeção", também amigo desde os tempos de colégio, merece aqui uma citação 
pelos inúmeros conselhos e interurbanos...

Por fim, gostaria de agradecer a parceira e também grande amiga Ariadne Olímpio pelo colo, pelas inúmeras palavras de apoio e carinho, além de seu companheirismo inestimável. 
Para minha vó Izabel (in memoriam), onde quer que ela esteja... 


\section{Resumo}

Cerca de $25 \%$ do conteúdo energético do universo se encontra sob uma forma de natureza ainda não determinada e é conhecida pelo nome de matéria escura. Desde as primeiras especulações acerca de sua existência (Zwicky 1933), vários modelos foram propostos na tentativa de justificar os dados observacionais encontrados mas, até hoje, nenhum deles foi capaz de cumprir essa tarefa a contento.

Nesta dissertação, apresentaremos uma breve discussão desses modelos, além de propor um novo, baseado na idéia de que tanto a matéria escura quanto a energia escura possam ser compostas pelo campo escalar de Born-Infeld. 


\begin{abstract}
Nearly twenty five percent of the energetic content of the universe appears in a form that is still unknown and is named dark matter. Since the first speculations about its existence (Zwicky $\sim 1933$ ), many models have been proposed trying to justify all the observed data but, until now, none of them has been able to solve this problem. In this monography, we will present a brief discussion about these models and propose a new one, based on the idea that both dark matter and dark energy could be the Born Infeld scalar field.
\end{abstract}


viii

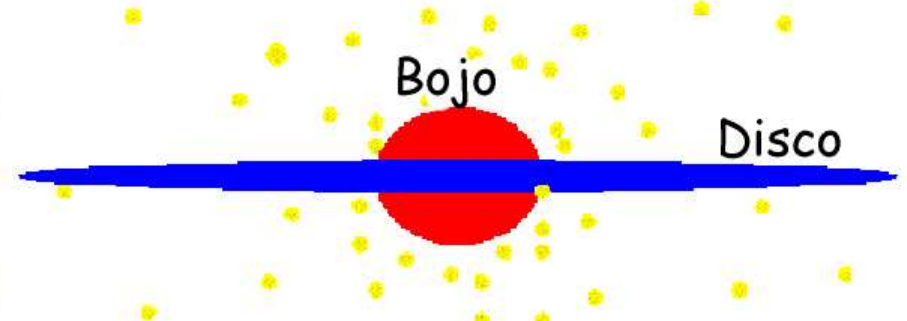

Halo

Figura 1: Representação esquemática das partes de uma galáxia típica. 


\section{Sumário}

1 Introdução 1

2 Modelo Cosmológico padrão $\quad 4$

2.1 Construção do modelo . . . . . . . . . . . . . . . . . 4

2.2 Evolução do fator de escala $a(t) \ldots \ldots \ldots . \ldots . \ldots 11$

2.3 Censo do universo . . . . . . . . . . . . . . . . 13

2.3.1 Idade do universo . . . . . . . . . . . . . 15

2.4 Distâncias em Cosmologia . . . . . . . . . . . . 15

2.5 Nucleossíntese primordial e a radiação cósmica de fundo . . . . . 17

2.5.1 Era da radiação . . . . . . . . . . . . . . 19

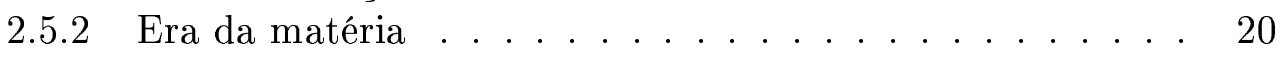

2.5.3 Radiação cósmica de fundo . . . . . . . . . . . . 20

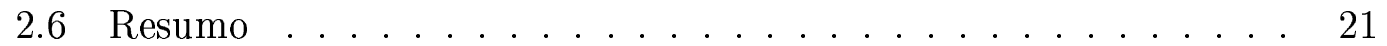

3 Matéria escura $\quad \mathbf{2 3}$

3.1 Evidências da necessidade da existência de matéria escura . . . . 24

3.2 Curvas de rotação . . . . . . . . . . . . . . . 26

3.2.1 Um pouco sobre a equação acolisional de Boltzmann . . . 28

3.3 Perfis de densidade . . . . . . . . . . . . . . . . 29

3.3.1 Alguns perfis populares . . . . . . . . . . . 31

3.3.1.1 Perfil N.F.W. . . . . . . . . . . . 31

3.3.1.2 Perfil Moore et al. . . . . . . . . . . . . 33

3.3.1.3 Halo pseudo-isotérmico . . . . . . . . . . . 33

3.3.2 Considerações sobre os perfis de densidade . . . . . . . . 34

3.4 Crise no modelo CDM devido ao perfil de densidades de halos escuros 35

3.5 Natureza da matéria escura . . . . . . . . . . . . 36

4 Modelagem dos halos $\quad 38$

4.1 Modelos com matéria escura fria . . . . . . . . . . . . . 38

4.1.1 O modelo do colapso esférico . . . . . . . . . . . . 38

4.1.2 Cálculos do modelo . . . . . . . . . . . . . . . . . . . 39

4.2 Modelos com campos escalares . . . . . . . . . . . . . 47

4.2.1 Halo escalar esférico em galáxias . . . . . . . . . . 47 
4.2.2 Modelo CDM X Modelo SFDM . . . . . . . . . . 47

4.2 .3 Modelos Mattos et al [?], [?] . . . . . . . . . . . . 49

4.2 .4 Modelo Arbey et al [?], [?] . . . . . . . . . . . . . 52

4.2 .5 Considerações gerais . . . . . . . . . . . . 53

4.2.6 Como um campo escalar pode simular a matéria escura . 53

4.2 .7 Discussões gerais . . . . . . . . . . . . 57

5 Campo escalar de Born-Infeld $\quad 67$

6 Halos de Born-Infeld $\quad \mathbf{7 5}$

6.1 Simplificação da métrica . . . . . . . . . . . . . . . . 75

6.2 Liberdade de gauge . . . . . . . . . . . . . . . . . . . . 76

6.3 Ansatz para o campo . . . . . . . . . . . . . . . . 77

6.4 Natureza das equações diferenciais . . . . . . . . . . . 77

6.5 Estudo do caso de alguns potenciais do tipo $U=U\left(\Phi, \Phi^{\dagger}\right) \ldots . .79$

6.5.1 Potencial do tipo $U\left(\Phi, \Phi^{\dagger}\right)=K \Phi^{p} \ldots \ldots \ldots \ldots$. . . . . . . 80

6.5.2 Potencial do tipo $U\left(\Phi, \Phi^{\dagger}\right)=e^{\beta|\Phi|} \ldots \ldots \ldots \ldots . . \ldots 84$

6.5.3 Potencial do tipo $U\left(\Phi, \Phi^{\dagger}\right)=e^{\beta|\Phi|^{2}} \ldots \ldots \ldots \ldots$. . . . . . . . . . 86

6.5.4 Potencial do tipo $U\left(\Phi, \Phi^{\dagger}\right)=e^{\beta|\Phi|^{3}} \ldots \ldots \ldots . \ldots 87$

6.6 Considerações finais . . . . . . . . . . . . . . 90

$\begin{array}{lll}7 & \text { Conclusões e perspectivas } & 92\end{array}$

A Espaço-tempo esfericamente simétrico $\quad 94$

$\begin{array}{ll}\text { B Universo de Einstein-de Sitter } & 97\end{array}$

$\begin{array}{ll}\text { C Abordagem via teoria linear de perturbações } & 99\end{array}$

$\begin{array}{lr}\text { D Tabela com as conexões } & 102\end{array}$

$\begin{array}{ll}\text { E Roteiro para continuação do trabalho } & 103\end{array}$

E.1 Possibilidades com um potencial do tipo exponencial . . . . . . 103

E.2 Como relacionar os modelos com campos escalares com os dados observacionais? . . . . . . . . . . . . . . 105 


\section{Lista de Figuras}

1 Representação esquemática das partes de uma galáxia típica. . . . . . . . . viii

2.1 Diagrama original de Hubble [?] e um resultado do Projeto HST [?] utilizando vários métodos diferentes de medida de distância. Conforme pode se observar, o melhor ajuste atual para os dados corresponde a uma constante de Hubble $\left(H_{0}\right)$ de aproximadamente $72 \mathrm{~km} \cdot \mathrm{Mpc}^{-1} \cdot \mathrm{s}^{-1} \ldots \ldots \ldots$

2.2 Linha de mundo (em vermelho) para o observador $\mathrm{O}$ e para a galáxia $\mathrm{G}$. As linhas azuis representam a trajetória dos raios de luz. . . . . . . . . .

2.3 Valores permitidos para $\Omega_{m} \equiv \Omega_{D M 0}+\Omega_{B 0}$ e $\Omega_{\Lambda}$, extraído de [?]. Os vínculos dizem respeito a dados dos experimentos Sloan Digital Sky Survey (SDSS) e WMAP, além de restrições provenientes de observações de supernovas. . . .

3.1 Extraído da referência [?], apresenta mais de 100 curvas de rotação diferentes, sugerindo fortemente um perfil plano para longas distâncias. . . . . . . .

4.1 Representação esquemática (bidimensional) da evolução das camadas. As camadas azuis já passaram pelo turn-around e agora apenas diminuem seu volume, o contrário valendo para as verdes. A camada vermelha, indicada por $\mathrm{j}$ no desenho, aparece no exato momento em que atinge $r_{\max } . \ldots \ldots$

4.2 Espectro das possíveis condições iniciais que levam a um decaimento da solução para a métrica $u(x)$ no infinito. Os picos se referem à condições iniciais que levam à soluções com comportamento assintótico decrescente para as curvas de rotação. . . . . . . . . . . . . . . . . . 64

4.3 Curvas de rotação para os autovalores $n=0$ e $n=1 . \quad \ldots \ldots .64$

4.4 Curvas de rotação para os autovalores $n=2$ e $n=3 \ldots \ldots$. . . . . . . . 65

4.5 Curvas de rotação para os autovalores autovalores $\mathrm{n}=5$ e $\mathrm{n}=10 \ldots \ldots 6$

4.6 Curva de rotação para o autovalor $n=\infty \ldots \ldots 66$

5.1 Gráfico de $\sigma(x)$ no caso de um potencial $U\left(\Phi, \Phi^{*}\right)$ constante para duas condições iniciais representativas do comportamento da função. Uma para uma condição inicial próxima de 0, outra próxima de 1 . Em ambos os casos, $\tilde{\sigma}^{\prime}(0)$ foi suposto nulo. Em cada gráfico, plotou-se também uma curva do tipo $\sigma(0) \frac{\sin (x)}{x}$, que averiguamos aproximar com relativa precisão a solução exata. . . . . . . . . 
5.2 Gráficos para a densidade de energia e pressões do campo. No caso apresentado, foi utilizado um $\widetilde{\sigma}(0)=0.999, \tilde{\sigma}^{\prime}(0)=0$ e o potencial $U\left(\Phi, \Phi^{*}\right)$, suposto constante, foi normalizado para 1.

5.3 Gráfico log-log da densidade de energia para várias condições iniciais diferentes . 73

5.4 Gráficos para $x^{2} \rho(x)$ para uma condição inicial qualquer representativa do comportamento da função $\left(\sigma(0)=0.50, \sigma^{\prime}(0)=0\right)$. Os gráficos mostram regiões distintas do espaço (próxima ou distante da origem) e as diferenças encontradas quando é descontado (esquerda) ou não o fundo.

6.1 Gráficos para a análise do modelo de [?, ?] apresentado no Cap. 4. Nos gráficos, as seguintes condições iniciais foram utilizadas: $\sigma(0)=1, \sigma^{\prime}(0)=0$, $u_{*}(0)=-1.3437, u_{*}^{\prime}(0)=0($ caso $n=2)$ e $\sigma(0)=1, \sigma^{\prime}(0)=0, u_{*}(0)=$ -1.6831 e $u_{*}^{\prime}(0)=0($ caso $n=10) \ldots \ldots \ldots \ldots \ldots$

6.2 Gráfico tridimensional apresentando um trecho do espaço de parâmetros para $u(x)$ e $p \ldots \ldots \ldots \ldots \ldots \ldots$

6.3 Gráficos para alguns valores do parâmetro $\mathrm{p}(p=0,1,2)$ do potencial para o campo. É imediato notar que TADS só se torna negativo (o que permitiria um comportamento do tipo exponencial para o campo) para valores muito grandes e positivos de $\mathrm{u}(x) . \ldots \ldots \ldots$

6.4 Gráficos da solução para o campo e da densidade efetiva para cada um dos casos. Os parâmetros utilizados foram $u(0)=-0.01, u^{\prime}(0)=0, \sigma(0)=0.2$, $\sigma^{\prime}(0)=0$ e o valor para $\frac{8 \pi G}{\omega^{2}} K=10^{-5} \ldots \ldots \ldots \ldots$

6.5 Curvas de rotação para as partículas-teste. As curvas foram feitas para uma região onde a aproximação newtoniana para $u(x)$ ainda é válida e os parâmetros utilizados foram os mesmos do gráfico (6.4). O painel inferior apresenta a evolução do parâmetro $u_{e f f}(x) \ldots \ldots \ldots \ldots \ldots$

6.6 Representação tridimensional do espaço do parâmetro $\beta$ e do coeficiente $u(x)$. À direita é apresentado um corte para um caso representativo, quando $\beta=1$. Novamente, TADS só se torna negativo para valores relativamente grandes de $u(x) \ldots \ldots \ldots \ldots \ldots \ldots \ldots$

6.7 Gráfico representativo da evolução dos parâmetros. Nesse caso em particular, $\beta=2 \ldots \ldots \ldots \ldots \ldots \ldots \ldots$

6.8 Representação dos possíveis valores para o parâmetro $\beta$ e as possíveis implicações para o sinal de TADS. O gráfico a direita é um exemplo representativo das conclusões obtidas, que também requerem um valor alto de $u(x)$ para que se obtenha um TADS negativo. . . . . . . . . . . . .

6.9 O gráfico à esquerda apresenta a solução $\sigma_{01}$ (cujo módulo sempre é > 1) como função de $\beta$. No gráfico à direita, plotaram-se parametricamente as partes real e imaginária das soluções $\sigma_{02}$ e $\sigma_{03}$. As setas indicam o sentido de crescimento de $|\beta| . \ldots \ldots \ldots \ldots \ldots \ldots \ldots \ldots$

6.10 Raízes da equação para $\sigma_{0} \ldots \ldots \ldots$. . . . . . . . . . . 89

$6.11 T A D S$ como função de $u \ldots \ldots$. . . . . . . . . . . 89

$6.12 \sigma$ como função de x para várias condições iniciais. . . . . . . . . . . . 90 
6.13 Comportamento possível para as curvas de rotação em um caso de potencial do tipo lei de potência, com $\mathrm{p}=0$ (potencial constante). . . . . . . . . . 91

E.1 Caso $V(\Phi)=e^{\beta \Phi^{2}}, \operatorname{com} \beta=0.5, \sigma(0)=0.02, \sigma^{\prime}(0)=0, u(0)=$ $-0.01, u^{\prime}(0)=0 \ldots \ldots \ldots \ldots \ldots \ldots$ 


\section{Lista de Tabelas}

2.1 Variação do fator de escala com o tempo para diferentes componentes dominantes. . . . . . . . . . . . . . . . . 12

3.1 Parâmetros (3.17) para alguns dos perfis mais utilizados para modelagem da densidade dos halos escuros em galáxias. Os valores de $\mathrm{R}\left(r_{m}, r_{s}\right.$ ou $\left.r_{c}\right)$ variam de galáxia para galáxia. . . . . . . . . . . . . 34 


\section{Capítulo 1}

\section{Introdução}

Temos hoje muito mais informações a respeito do universo do que provavelmente o mais otimista dos gregos antigos poderia imaginar. A crescente avalanche de novos dados observacionais que vêm surgindo a cada dia começa a permitir a montagem de uma "visão panorâmica" do assunto que até pouco tempo atrás era simplesmente inimaginável. Resultados de novos experimentos são divulgados constantemente, o que nos permite descrever com razoável eloqüência o que entendemos por modelo cosmológico padrão (MCP). Esse modelo, por sua vez, tem se mostrado como uma espécie de rascunho de idéias e constatações que vai se aperfeiçoando a cada novo experimento lançado, alçando a Cosmologia de uma ciência bastante especulativa para um reconhecido status de teoria amplamente amparada em resultados observacionais.

Por outro lado, talvez ainda mais interessante que o crescente volume de dados coletados é que praticamente na mesma medida em que surgem novas informações, como por exemplo medidas da radiação cósmica de fundo e suas anisotropias [?], dados de supernovas [?, ?, ?, ?, ?], medidas quantitativas da lei de Hubble [?, ?, ?, ?] ou curvas de rotação de galáxias [?], um sem-número de modelos é proposto para tentar explicar as observações (matéria escura, energia escura, quintessência, quartessência, constante cosmológica, branas, dimensões extras, dentre tantos outros), o que já fornece bons indícios tanto da riqueza e da complexidade do assunto quanto do muito que ainda precisa ser descoberto.

Na realidade, essa situação aparentemente confusa reflete o fato de que atualmente a Cosmologia se encontra em um período crucial de seu desenvolvimento. São muitas as possibilidades a serem testadas e modeladas. Por exemplo, temos no momento boas razões para crer que nosso universo se encontra em um estado de expansão acelerada [?]. Somos capazes também de tecer comentários coerentes a respeito da abundância de vários elementos leves [?], além de podermos afirmar com relativa confiança que a densidade média de energia no universo é igual à densidade crítica, o que o obriga, na linguagem da Relatividade Geral, a ter uma seção espacial plana, com curvatura nula [?].

Por outro lado, todo esse suposto sucesso carrega consigo novos e profundos 
questionamentos. Por exemplo, atualmente também já sabemos que apenas cerca de $5 \%$ do que se acredita ser o conteúdo total de energia do universo [?, ?] se encontra na forma de matéria ordinária, bariônica. Isso obviamente gera uma dúvida sobre o que poderia ser a porção restante. A idéia com maior aceitação hoje afirma que cerca de $70 \%$ do conteúdo energético (total) deve se encontrar na forma da chamada energia escura, que seria responsável pela expansão acelerada do universo. Os restantes $25 \%$ estariam na forma de uma componente intitulada matéria escura, que apenas interage de maneira gravitacional e teria efeitos mais marcantes em pequenas escalas (da ordem de $10 \mathrm{Mpc}$ ), sendo praticamente indispensável em cenários de formação de estruturas [?].

Esse cenário de dúvidas parece então deixar bastante claro que respostas definitivas para todas as perguntas intrigantes acerca da natureza do universo com certeza ainda não surgiram. Por outro lado, é verdade patente que a ciência precisa investigar o maior número de possibilidades para poder crescer. Confrontar especulações com dados experimentais, formular hipóteses, contestar teorias. Neste trabalho, buscar-se-á dar uma pequena colaboração nesse sentido, analisando-se um problema ainda não respondido a contento em Cosmologia: o problema dos halos de matéria escura em galáxias. Esses halos seriam estruturas com presença marcante em praticamente todos os tipos de galáxias e aglomerados ${ }^{1}$, notadamente em galáxias espirais e que poderiam por exemplo responder pelo perfil plano observado de algumas curvas de rotação (velocidades circulares constantes mesmo para locais bastante afastados do centro galáctico). Para tanto, acrescentou-se ao estudo de alguns modelos propostos na literatura [?, ?, ?, ?, ?, ?] um trabalho fenomenológico em cima da hipótese desses halos poderem ser formados por campos escalares não-canônicos, notadamente os descritos pela Lagrangeana de Born-Infeld.

Optou-se pela seguinte divisão para a exposição dessa dissertação: segue esta introdução um capítulo versando sobre o modelo cosmológico padrão, suas implicações e pontos ainda não esclarecidos. Também são discutidas algumas definições amplamente utilizadas em cosmologia. O Cap. 3 foca o porquê da necessidade da existência da matéria escura, apontando algumas possibilidades para sua natureza (algumas já descartadas, outras não), além de fazer breves comentários sobre alguns perfis de densidade para os halos de matéria escura obtidos via simulações numéricas, como é o caso do NFW [?] e o de Moore et al [?]. O quarto capítulo expõe alguns modelos estudados e que são propostos na literatura para tentar justificar o comportamento observado dos halos, como o modelo do colapso esférico não-linear, além de alguns utilizando campos escalares. É dado particular enfoque aos modelos de [?] e [?, ?], que serviram como base e foram grande fonte de inspiração para nosso trabalho. O quinto capítulo engloba o nosso método, explica como foi construído o modelo com a Lagrangeana de

\footnotetext{
${ }^{1}$ Para um confronto de idéias com relação às galáxias elípticas, sugere-se ao leitor interessado a leitura das referências [?, ?, ?].
} 
Born-Infeld e fornece uma espécie de guia de como foram realizados os cálculos. O Cap. 6 apresenta os resultados encontrados, fazendo comparações entre os diversos modelos. No sétimo capítulo apresentamos a conclusão, com perspectivas futuras para o trabalho.

Neste texto, será utilizado o chamado sistema de unidades naturais, onde $c=k_{b}=\hbar=1$. A métrica utilizada no decorrer dos capítulos terá assinatura $(+,-,-,-)$. As demais convenções são as usuais: índices gregos variam de 0 a 3 , índices latinos de 1 a 3 e será utilizada a notação de somatória de Einstein, onde índices repetidos subentendem soma. 


\section{Capítulo 2}

\section{Modelo Cosmológico padrão}

O advento da Teoria da Relatividade Geral de Einstein no ano de 1916 forneceu aos cosmólogos uma possibilidade real de descrever o universo ancorados em bases bastante sólidas ${ }^{1}$. Dessa época até agora, pode-se afirmar com propriedade que progrediu-se muito, não só do ponto de vista teórico e interpretativo mas também no que tange à evolução dos aparelhos de medida e observação. Muitas descobertas foram feitas e podemos hoje, com razoável grau de confiança, ter a ousadia de propor um modelo cosmológico padrão (MCP) baseado nesse conhecimento acumulado ao longo dos últimos anos.

O modelo tido hoje como padrão na cosmologia é muitas vezes referenciado na literatura como modelo do Big Bang quente, segundo o qual o universo evoluiu durante bilhões de anos de um estado muito mais denso e quente até a situação em que se encontra hoje. Numa área que por tanto tempo foi tão especulativa, é no mínimo curioso que algum modelo tenha conseguido se destacar a ponto de atingir um status de quase unanimidade perante a comunidade científica. Entretanto, tal sucesso é justificável. O acúmulo de dados observacionais conseguidos nas últimas décadas finalmente pôde colocar a prova as mais diversas teorias e selecionar as que se mostravam mais consistentes com as observações. No caso da teoria do "Big-Bang", os sucessos podem ser resumidos em três pilares básicos: o diagrama de Hubble exibindo a expansão do universo, a abundância de elementos leves de acordo com as previsões da nucleossíntese primordial e a constatação da existência da radiação cósmica de fundo.

\subsection{Construção do modelo}

Como poderia ser o universo e como ele é de fato? Teoricamente, seria possível existir um sem-número de possibilidades para o nosso universo. Entretanto, o apelo para o princípio cosmológico acaba simplificando e diminuindo sobremaneira as situações plausíveis, embora ele, por si só, ainda não seja capaz de fornecer uma

\footnotetext{
${ }^{1}$ Informações sobre cosmologia pré-relativística podem ser encontradas por exemplo em [?].
} 
solução única para a questão. Veremos a seguir um esboço dos virtuais caminhos que o universo poderia tomar e as razões pelas quais damos preferência a algumas hipóteses sobre a evolução cósmica em detrimento de outras.

\section{O princípio cosmológico e a métrica FRW}

A construção de um modelo cosmológico bem sucedido esbarra primeiramente em uma questão bastante delicada, a de como utilizar o maquinário fornecido pela Relatividade Geral levando em consideração o universo inteiro. A aplicação da teoria de Einstein (ou mesmo a newtoniana) seria com certeza uma aventura desprovida de muitas esperanças se não fosse pela formulação de uma hipótese simplificadora ${ }^{2}$ bastante forte: o universo é homogêneo e isotrópico em largas escalas, ou seja inexistem direções (isotropia) ou pontos (homogeneidade) privilegiados no universo. Tal hipótese, por sua vez, carrega consigo uma peculiaridade: a existência de certos observadores privilegiados que realmente vêem essa condição, ou seja, para os quais o universo é de fato homogêneo e isotrópico. Esses observadores são aqueles que se encontram em repouso com relação à distribuição local de matéria e são chamados de comóveis. Por simplicidade, opta-se então pela descrição do universo em termos deles, procurando por uma métrica para o espaço-tempo escrita em um sistema de coordenadas $\left(t, x^{i}\right)$ condizente com a condição privilegiada dos mesmos. Pode-se provar que o elemento de linha $d s^{2}$ para um desses observadores tem a forma [?, ?, ?, ?]:

$$
d s^{2}=d t^{2}-\sigma_{i j} d x^{i} d x^{j} \equiv d t^{2}-d l^{2},
$$

o elemento $g_{00}$ igual a 1 pela necessidade de termos a igualdade $d s^{2}=d t^{2}$ para observadores comóveis (com $x^{i}$ constante). Para responder pelas propriedades de homogeneidade e isotropia, a porção espacial da métrica deve descrever um espaço de curvatura constante. Um espaço dessa natureza apresenta um tensor de Riemann:

$$
S_{i j k l}=c\left(\sigma_{i k} \sigma_{j l}-\sigma_{i l} \sigma_{k j}\right),
$$

onde $6 c=\sigma^{i k} \sigma^{j l} S_{i j k l}=S$. O 3-espaço mais geral com essas propriedades pode por sua vez ser descrito por um elemento de linha na forma:

$$
d l^{2}=a^{2}(t)\left[\frac{d r^{2}}{1-k r^{2}}+r^{2}\left(d \theta^{2}+\sin ^{2} \theta d \phi^{2}\right)\right],
$$

com $k=0,+1$, ou -1 (respectivamente universo plano, fechado ou aberto ) e $a(t)$ uma função apenas da coordenada temporal, conhecida também pelo nome de fator de escala e relacionada com a curvatura por $S=\frac{6 k}{a^{2}}$. Os valores permitidos

\footnotetext{
${ }^{2}$ Também conhecida como uma variação do chamado princípio copernicano (ou cosmológico).
} 
para k estão intrinsecamente relacionados à quantidade de matéria no universo, como poderá ser visto futuramente no tópico sobre dinâmica no MCP.

Esse resultado permite escrever a métrica completa para espaços homogêneos e isotrópicos, também conhecida pelo nome de métrica de Friedmann RobertsonWalker (FRW):

$$
d s^{2}=d t^{2}-a^{2}(t)\left[\frac{d r^{2}}{1-k r^{2}}+r^{2}\left(d \theta^{2}+\sin ^{2} \theta d \phi^{2}\right)\right] .
$$

Considerações iniciais feitas, podemos agora analisar algumas conseqüências físicas de termos uma métrica do tipo FRW. A primeira delas é que qualquer distância física (própria) mudará com o tempo proporcionalmente a $a(t)$. Uma maneira usual de se notar isso é raciocinar em cima da seguinte situação: imaginemos um triângulo definido pelas posições de três observadores comóveis. $\grave{A}$ medida que o tempo passa, é de se esperar que esse triângulo possa mudar seu tamanho, mas em hipótese alguma sua forma, pois isso implicaria na existência de alguma direção privilegiada no espaço (o que violaria a isotropia). Definindo $l(t)$ como a distância física (própria) separando dois desses observadores, devemos ter então:

$$
l(t)=a(t) l\left(t_{0}\right),
$$

onde é comum tomarmos $a\left(t_{0}\right)=1$, com $t_{0}$ denotando o instante atual.

Olhando para (2.4), chegamos à evidente conclusão de que a separação física entre dois observadores comóveis poderá obviamente mudar com o passar do tempo, bastando para isso que o fator de escala varie. Dessa forma, esses mesmos observadores conseguem atribuir um ao outro uma velocidade relativa ${ }^{3}$ dada por:

$$
v(t)=\frac{d l}{d t}=l\left(t_{0}\right) \dot{a}(t)=l(t) \frac{\dot{a}(t)}{a(t)} \equiv l(t) H(t) .
$$

com $H(t)=\frac{\dot{a}(t)}{a(t)}$ conhecido pelo nome de parâmetro de Hubble.

É imperativo apontar que este resultado casou-se perfeitamente com algumas observações que já haviam sido feitas por volta de 1929 pelo astrônomo inglês Edwin Hubble. Medindo o espectro de galáxias espirais, ele foi capaz de encontrar um curioso padrão de linhas espectrais atômicas que apresentavam um recorrente desvio para o vermelho (redshift). Embora seus resultados quantitativos estivessem bastante incorretos, a interpretação dada por ele na época foi a de que a velocidade das galáxias estudadas estava aumentando linearmente com a distância $l$, fato comprovado pela relação (2.5).

Pela figura (2.1) podemos observar que esse resultado (um fator de escala variando no tempo) permitido pelo modelo padrão e, conforme citado, já constatado

\footnotetext{
${ }^{3} \mathrm{Na}$ realidade, uma expressão mais formal implicaria na possibilidade de os observadores medirem também uma velocidade peculiar $v_{p}=\dot{l}\left(t_{0}\right) a(t)$ que deveria ser acrescida à $v(t)$. Aqui ela foi suposta zero.
} 
há mais de 60 anos, é de fato real e reflete a idéia que temos hoje de estarmos em um universo em expansão.
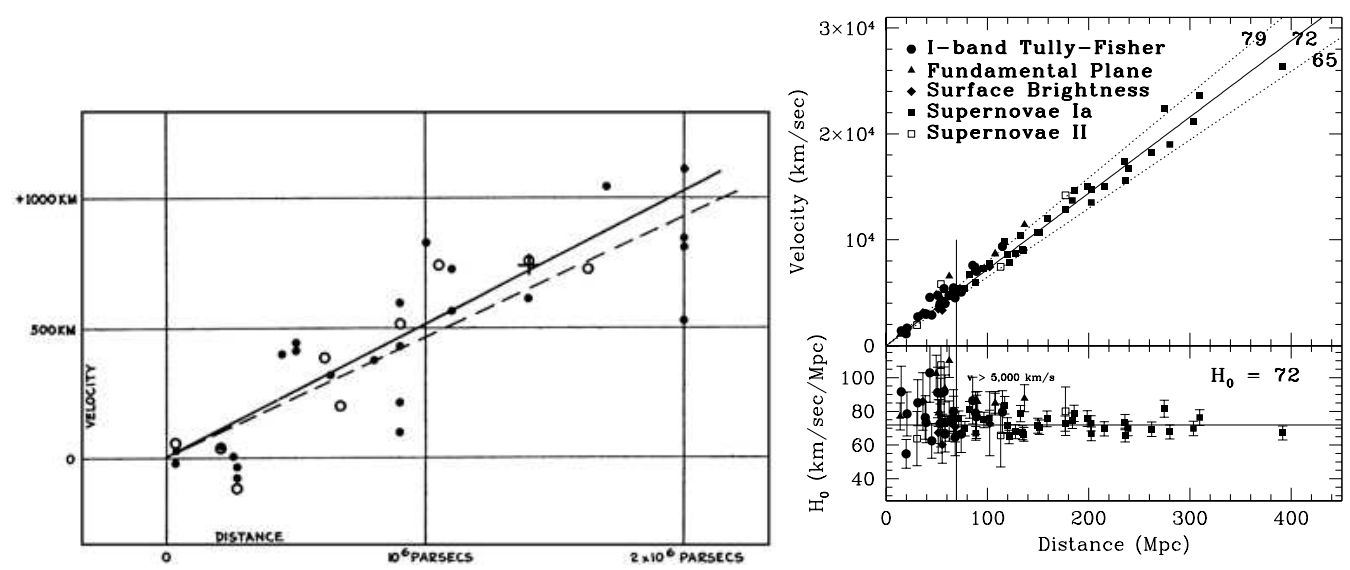

Figura 2.1: Diagrama original de Hubble [?] e um resultado do Projeto HST [?] utilizando vários métodos diferentes de medida de distância. Conforme pode se observar, o melhor ajuste atual para os dados corresponde a uma constante de Hubble $\left(H_{0}\right)$ de aproximadamente $72 k m \cdot M p c^{-1} \cdot s^{-1}$.

Por se tratar de algo que aparece com freqüência na literatura, é importante mencionar que existe uma notação bastante usual que escreve o parâmetro de Hubble na forma $H\left(t=t_{0}\right)=H_{0}=100 h \mathrm{Kms}^{-1} \mathrm{Mpc}^{-1}$. Uma das medidas mais recentes (via dados da radiação cósmica de fundo) parecem apontar para um $\mathrm{h}$ em torno de $0.71 \pm 0.04$ [?].

\section{Um pouco sobre a propagação da luz em cosmologia relativística e o redshift cosmológico}

Em cosmologia relativística, é assumido que a luz se propaga da maneira descrita pela relatividade geral. Sendo assim, olhemos para uma métrica do tipo FRW (2.3) e tentemos compreender de que maneira um observador $(\mathrm{O})$ recebe a luz de uma galáxia distante qualquer.

Sabemos que os fótons devem viajar segundo geodésicas nulas, o que se traduz nas seguintes condições (para geodésicas radiais):

$$
d s^{2}=d \theta=d \phi=0 .
$$

Por sua vez, (2.6) utilizada na métrica de FRW, nos fornece:

$$
\frac{d t}{a(t)}= \pm \frac{d r}{\sqrt{1-k r^{2}}},
$$


sendo que o sinal mais representa um raio de luz se afastando e o menos um raio de luz se aproximando do observador.

Consideremos agora um raio de luz emitido por uma galáxia $\mathrm{G}$ com linha de mundo $r=r_{1}$ no instante de tempo $t=t_{1}$ e recebido por 0 (suposto na origem das coordenadas, ou seja, com $\mathrm{r}=0$ ) no instante $t=t_{0}$. De (2.7), teremos:

$$
\begin{gathered}
\int_{t_{1}}^{t_{0}} \frac{d t}{a(t)}=-\int_{r_{1}}^{0} \frac{d r}{\sqrt{1-k r^{2}}}=f\left(r_{1}\right), \\
f\left(r_{1}\right)=\left\{\begin{array}{ccc}
\sin ^{-1} r_{1} & p / & k=1 \\
r_{1} & p / & k=0 \\
\sinh ^{-1} r_{1} & p / & k=-1
\end{array} .\right.
\end{gathered}
$$

Um resultado mais interessante pode ser extraído se considerarmos agora não um, mas dois raios de luz sucessivos provenientes de $\mathrm{G}$, por exemplo originados nos instantes $t_{1}$ e $t_{1}+d t_{1}$, sendo recebidos em 0 nos instantes $t_{0}$ e $t_{0}+d t_{0}$. A figura a seguir ajuda a melhor ilustrar a situação:

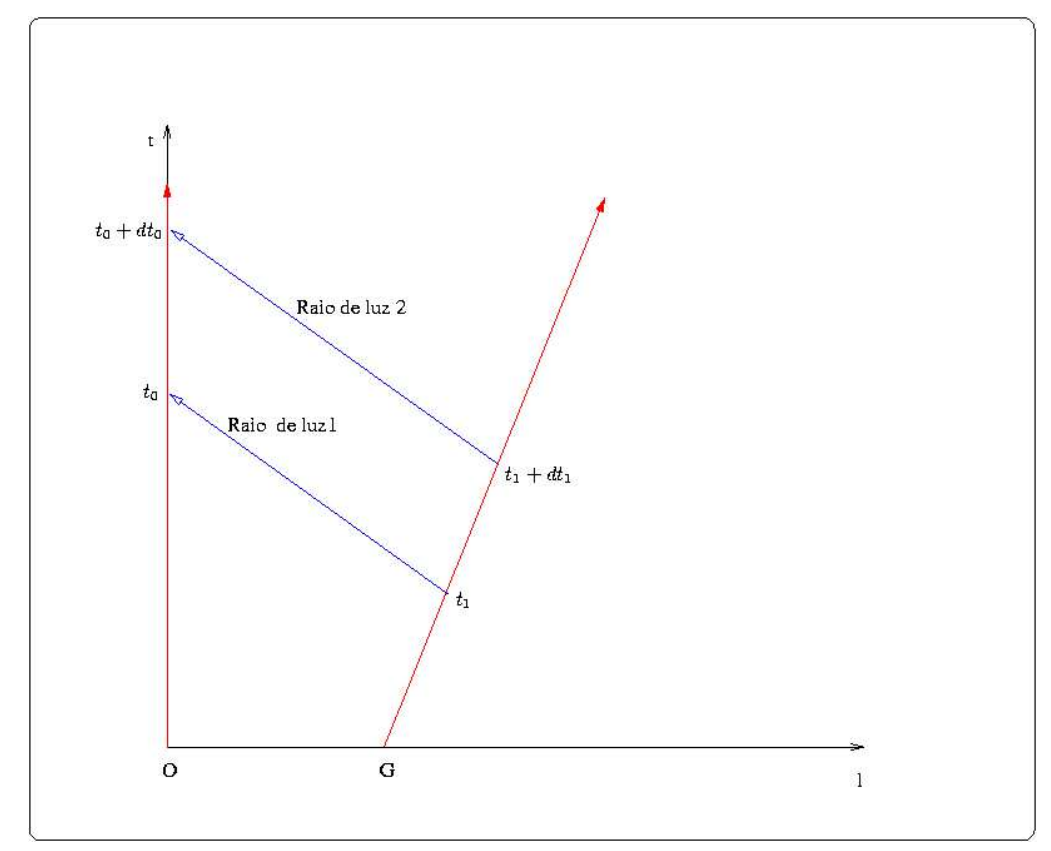

Figura 2.2: Linha de mundo (em vermelho) para o observador O e para a galáxia G. As linhas azuis representam a trajetória dos raios de luz.

Prosseguindo, de (2.8), ficamos com:

$$
\int_{t_{1}+d t_{1}}^{t_{0+d t_{0}}} \frac{d t}{a(t)}=\int_{t_{1}}^{t_{0}} \frac{d t}{a(t)},
$$


a igualdade vindo do fato de ambas as integrais serem iguais a $f\left(r_{1}\right)$. Podemos ainda manipular um pouco essa última expressão obtendo:

$$
\int_{t_{1}+d t_{1}}^{t_{0+d t_{0}}} \frac{d t}{a(t)}-\int_{t_{1}}^{t_{0}} \frac{d t}{a(t)}=\int_{t_{0}}^{t_{0}+d t_{0}} \frac{d t}{a(t)}-\int_{t_{1}}^{t_{1}+d t_{1}} \frac{d t}{a(t)}=0 .
$$

Assumindo por hipótese que $a(t)$ pode ficar sem variar muito durante os intervalos $d t_{1}$ e $d t_{0}$, supostos pequenos, conseguimos resolver as últimas integrais acima e escrever:

$$
\frac{d t_{0}}{a\left(t_{0}\right)}=\frac{d t_{1}}{a\left(t_{1}\right)}
$$

Lembrando que para observadores comóveis temos $r, \theta$ e $\phi$ constantes, podemos afirmar que, novamente de $(2.3), d s^{2}=d t^{2}$, ou seja, $d t$ mede o tempo próprio para esses observadores. Resumindo, enquanto para um observador em G se passou um intervalo igual a $d t_{1}$, para outro localizado em 0 o intervalo medido será de $d t_{0}=\frac{a\left(t_{0}\right)}{a\left(t_{1}\right)} d t_{1}$. Este resultado, por sua vez, motiva a definição de uma outra grandeza conhecida como redshift $(\mathrm{z})^{4}$ :

$$
1+z \equiv \frac{\nu_{1}}{\nu_{0}}=\frac{a\left(t_{0}\right)}{a\left(t_{1}\right)}
$$

$\nu_{1}$ e $\nu_{0}$ sendo as freqüências medidas dos fótons emitido e recebido, respectivamente. Um bom modo de se convencer da última igualdade em (2.10) é perceber que a dilatação para os intervalos de tempo em (2.9) (caso do universo em expansão, com $\left.a\left(t_{0}\right)>a\left(t_{1}\right)\right)$ se reflete também numa diferença para a medida da freqüência dos fótons pelo observador e pelo emissor.

Apenas para efeito de completeza, é conveniente afirmar que muitas vezes parece ocorrer uma certa confusão entre o redshift cosmológico (devido ao efeito de expansão do universo) e o chamado desvio Doppler (causado pela diferença de velocidade relativa entre duas fontes). Para redshifts pequenos, a identificação entre os dois é razoavelmente clara $\left(z=\frac{v}{c}\right.$, lembrando que tomamos $\mathrm{c}=1$ no nosso texto) mas, para grandes redshifts, isso não se aplica. Ao leitor interessado, é sugerida a leitura da discussão sobre vários conceitos controversos existente na seção 3.3 da referência [?].

\section{Considerações sobre a dinâmica do MCP}

Como é sabido da relatividade geral, a equação de Einstein nos fornece uma maneira de relacionar o conteúdo de energia com a geometria do espaço:

$$
G^{\mu}{ }_{\nu}=R^{\mu}{ }_{\nu}-\frac{1}{2} \delta^{\mu}{ }_{\nu} R=8 \pi G T^{\mu}{ }_{\nu}
$$

\footnotetext{
${ }^{4}$ No caso em que as fontes estão se aproximando, ele freqüentemente é chamado blueshift.
} 
onde $T^{\mu}{ }_{\nu}$ é o chamado tensor de momento-energia que, no caso cosmológico, deve conter as informações a respeito do conteúdo de matéria e energia do universo. O tensor de momento e energia mais simples que se pode imaginar para o universo (e exatamente o que é utilizado) é o de um fluido perfeito [?]. Na realidade, uma maneira alternativa de chegarmos à mesma conclusão é apelando para o chamado postulado de Weyl. Em termos simples, Weyl afirma que existe uma espécie de "substrato", um fluido permeando o espaço e dentro do qual as galáxias se movem como "partículas fundamentais". Isso é razoável, já que em grandes escalas elas podem ser tomadas com boa aproximação como partículas puntuais, com velocidades peculiares pequenas, provavelmente apenas importantes no trato com considerações astrofísicas locais. Uma forma mais precisa de enunciar seu postulado entretanto é:

"As partículas do substrato repousam no espaço-tempo em uma congruência de geodésicas do tipo tempo, divergindo de algum ponto no passado infinito ou no futuro infinito" [?].

Prosseguindo, recorrendo novamente à hipótese de homogeneidade e isotropia, algumas simplificações podem ser conseguidas para a equação de Einstein. A primeira delas é que devemos ter $T_{0}^{i}=T^{j}{ }_{k}(j \neq k)=0$, já que um observador comóvel "não vê" densidades de momento para esse fluido e também não mede anisotropias de pressão. Além disso, as componentes espaciais da diagonal principal devem ser idênticas. Essas constatações resultam em:

$$
T_{\nu}^{\mu}=\operatorname{diag}(\rho(t),-p(t),-p(t),-p(t)),
$$

onde $\rho$ é a densidade de energia (total) e p a pressão (total) do fluido. Utilizando a métrica FRW (2.3) para escrever o tensor de Einstein, combinando com (2.12) e utilizando (2.11), chegamos às chamadas equações de Friedmann:

$$
\begin{aligned}
\frac{\dot{a}^{2}+k}{a^{2}} & =\frac{8 \pi G}{3} \rho, \\
\frac{2 \ddot{a}}{a}+\frac{\dot{a}^{2}+k}{a^{2}} & =-8 \pi G p .
\end{aligned}
$$

As equações (2.13) e (2.14) podem ser utilizadas conjuntamente com uma equação de estado para o fluido perfeito, algo do tipo $p=p(\rho)$, o que permite determinar completamente as funções $a(t), \rho(t)$, e $p(t)$. Além disso, da primeira das equações (2.13), somos capazes de extrair uma expressão para a curvatura das seções espaciais do espaço-tempo:

$$
\frac{k}{a_{0}^{2}}=\frac{8 \pi G}{3} \rho_{0}-H_{0}^{2} \equiv H_{0}^{2}\left(\Omega_{0}-1\right)
$$

$\operatorname{com} \Omega_{0} \equiv \frac{\rho_{0}}{\rho_{c}}$ e a chamada densidade crítica $\rho_{c}$ dada por: 


$$
\rho_{c} \equiv \frac{3 H_{0}{ }^{2}}{8 \pi G} \simeq 1.0610^{4} h^{2} \mathrm{eV} . \mathrm{cm}^{-3},
$$

novamente o índice 0 indicando quantidades medidas no instante atual.

Dessa maneira, a equação (2.15) determina univocamente se teremos um universo plano $(\mathrm{k}=0)$, aberto $(\mathrm{k}=-1)$ ou fechado $(\mathrm{k}=+1)$, bastando para isso que saibamos a razão entre a densidade total de matéria que preenche o universo e a densidade crítica.

Da combinação das equações (2.13) e (2.14), é possível ainda obter um outro resultado bastante interessante e que carrega consigo gigantescas implicações:

$$
\frac{\ddot{a}}{a}=-\frac{4 \pi G}{3}(\rho+3 p)
$$

Da expressão (2.16), podemos facilmente perceber que a segunda derivada do fator de escala será sempre negativa quando estivermos lidando com um universo preenchido com matéria ordinária, comum, com pressão positiva (lembrando que $\rho$ representa a densidade total de energia no universo, proveniente de várias contribuições). Entretanto, conforme já anteriormente citado e como pode ser constatado em diversas referências [?, ?, ?, ?, ?], sabemos que nosso universo hoje se encontra em um estado de expansão acelerada. Esse resultado é absolutamente crucial e reflete a necessidade de termos em (2.16) pelo menos uma componente que contribua para a densidade e que tenha uma pressão negativa (pelo menos $<$ que $-\frac{1}{3} \rho$ ), o que poderia justificar os resultados observados.

Em resumo, nesse ponto surge uma das grandes complicações da Cosmologia moderna. Como conciliar teoria e observações de maneira coerente, ou seja, justificar a expansão acelerada do universo sem desestruturar completamente o modelo FRW? A proposta mais difundida hoje é que deva existir alguma componente no fluido cósmico, denominada genericamente "energia escura" que responda por essas propriedades. Por enquanto, a idéia que tem tido maior aceitação da comunidade científica, a que fornece o chamado "best fit" (melhor ajuste para os dados experimentais) [?] consiste no acréscimo à densidade de energia total do universo $\rho$ de uma contribuição devido a uma componente com pressão negativa intitulada constante cosmológica $(\Lambda)^{5}$, com uma densidade dada por $\rho_{\Lambda}=\frac{\Lambda}{8 \pi G}$. Na realidade, essa não é a única possibilidade, existe um bom número de modelos alternativos que tentam resolver essa questão [?, ?].

\subsection{Evolução do fator de escala $a(t)$}

Outro resultado importante que pode ser extraído via manipulação das equações de Friedmann (2.13), (2.14) diz respeito à evolução das várias componentes do

\footnotetext{
${ }^{5} \mathrm{Na}$ realidade, um termo desta natureza já havia aparecido na Cosmologia só que em um outro contexto, sugerido por Einstein, buscando obter soluções para um universo estático.
} 
fluido cósmico no decorrer da história do universo. A expressão da conservação $\left(\nabla_{\mu} T^{\mu \nu}\right)$ do tensor de momento e energia (2.12) para o fluido cósmico fornece:

$$
\frac{\partial \rho}{\partial t}+3 \frac{\dot{a}}{a}(\rho+p)=0 .
$$

A equação (2.17) pode ser resolvida se, por exemplo, soubermos qual a dependência funcional entre $\rho$ e $p$, ou seja, a equação de estado para o fluido. A abordagem usual é supor uma equação de estado para cada contribuinte da densidade total de energia do universo na forma:

$$
p_{i}=\omega_{i} \rho_{i},
$$

onde $\omega_{i}$ é uma constante, cujo valor varia de componente para componente e i é um índice que dá conta das várias contribuições diferentes. Injetando (2.18) em (2.17) e realizando a integração, podemos determinar como o fator de escala varia com o tempo quando cada um dos possíveis constituintes de (2.12) é dominante ${ }^{6}$ :

$$
a(t) \propto t^{\frac{2}{3\left(1+\omega_{i}\right)}}
$$

No caso de um universo dominado por matéria fria (não relativística), é uma boa aproximação supormos $\omega_{m}=0$ (pressão nula), o que, injetado em (2.17), permite escrever $a(t) \propto t^{\frac{2}{3}}$. Já para o caso de um universo dominado por radiação, $\omega_{r}=\frac{1}{3}$, resultando $a(t) \propto t^{\frac{1}{2}}$.

Podemos ainda olhar o problema sob outra ótica e, da mesma forma, fazer a análise da variação da densidade dessas componentes com o tempo, o que é resumido na tabela a seguir, que ainda acrescenta o caso da constante cosmológica:

\begin{tabular}{|c|c|c|}
\hline Componente dominante & $\omega_{i}$ & $\rho(t) \propto$ \\
\hline \hline Matéria $(\mathrm{m})$ & 0 & $a^{-3}$ \\
\hline Radiação(r) & $\frac{1}{3}$ & $a^{-4}$ \\
\hline Constante cosmológica $(\Lambda)$ & -1 & constante \\
\hline
\end{tabular}

Tabela 2.1: Variação do fator de escala com o tempo para diferentes componentes dominantes.

$\mathrm{Na}$ realidade, essa hipótese (dominação de alguma componente em alguma época) é de fato observada, hoje acreditamos que esses momentos de fato ocorreram ao longo da história do universo. Da tabela (2.1) observamos que a densidade de radiação decresce mais rapidamente que a densidade de matéria, o que nos permite intuir que, se em algum momento recente a matéria foi dominante, em algum período no passado a radiação era mais importante, $z_{e q}$ marcando a transição entre essas duas eras. Se igualarmos as densidades de energia para

\footnotetext{
${ }^{6}$ Ressalta-se aqui o detalhe de as componentes serem supostas evoluindo de maneira independente, de tal modo que 2.17 valha para cada uma delas.
} 
a matéria e a radiação e, lembrando da definição de redshift (2.10), podemos concluir:

$$
\rho_{m}=\rho_{c} \Omega_{m}(1+z)^{3}=\rho_{r}=\rho_{c} \Omega_{r}(1+z)^{4} \Rightarrow\left(1+z_{e q}\right)=\frac{\Omega_{m}}{\Omega_{r}}
$$

Da mesma maneira, podemos relacionar a temperatura dos fótons de radiação com o redshift. Utilizando a relação de Boltzmann, $E=k T_{r}$, onde E é a energia, $\mathrm{k}$ a constante de Boltzmann (de valor 1, utilizando o sistema natural de unidades), $T_{r}$ a temperatura da radiação, lembrando que $E=h \nu$ e, novamente utilizando (2.10), temos:

$$
T_{r} \propto a^{-1}
$$

o que implica

$$
T_{e q}=T_{0}\left(1+z_{e q}\right) .
$$

A expressão (2.22) expressa assim a já mencionada idéia: para $T \ll T_{e q}$, a densidade de energia é dominada por radiação, $\operatorname{com} p=\frac{1}{3} \rho$. Com o decorrer da evolução cósmica, a densidade de energia passa a ser dominada por matéria $(p \approx 0)^{7}$.

\subsection{Censo do universo}

As considerações feitas até aqui ainda não apresentaram muitos detalhes de quais são os termos que de fato aparecem contribuindo para a densidade de energia $\rho \mathrm{em}$ (2.12). Parece razoável supormos termos do tipo matéria, radiação e, depois da discussão posterior à (2.16), algum termo referente à energia escura. A pergunta subseqüente é qual seria a porcentagem de contribuição de cada um desses termos? Estimativas recentes [?] sugerem o seguinte (para as quantidades atuais): uma contribuição $\Omega_{R 0} \sim 10^{-4}$, proveniente de fótons da RCF mais neutrinos de baixa massa, matéria bariônica contribuindo com $\Omega_{B 0} \sim 5.10^{-2}$ e energia escura (aqui suposta constante cosmológica) com $\Omega_{\Lambda 0} \sim 7.10^{-1}$. É usual ainda trabalhar com (2.15) deixando-a na forma:

$$
1=\Omega_{0}+\Omega_{K 0},
$$

o que permite definir uma espécie de densidade de curvatura: $\Omega_{K 0}=\frac{-3 k}{8 \pi G a_{0}{ }^{2} \rho_{c}} \sim 0$ pelas observações, indicando, conforme já citado, um universo com seção espacial plana.

\footnotetext{
${ }^{7} \mathrm{Na}$ verdade, sabemos que a situação observada hoje é ligeiramente diferente, e a componente dominante é de energia escura.
} 
Coletados os resultados apresentados, uma tentativa de reescrever (2.15) entretanto fornece:

$$
\Omega_{K 0}+\Omega_{R 0}+\Omega_{B 0}+\Omega_{\Lambda 0} \neq 1
$$

um indício claro de que a contagem apresentada do conteúdo energético do universo ainda não está completa. Conforme será melhor esmiuçado no capítulo seguinte, as observações atuais sugerem ainda a existência de uma outra componente escura, esta intitulada matéria escura, com $\Omega_{D M 0} \sim 0.2$. Na realidade, se formos um pouco mais cuidadosos com as definições, é conveniente afirmar que podemos ter uma porção escura dentro da parcela bariônica, de modo que $\Omega_{D M 0}$ responde apenas pela parcela não-bariônica da matéria escura, o que será discutido com mais detalhes no capítulo seguinte.

Esse modelo de universo, cujas componentes mais relevantes são as indicadas em (2.23) acrescidas de $\Omega_{D M 0}$ (suposta matéria fria, não relativística) é conhecido hoje pelo nome de $\Lambda C D M$, em alusão à suas componentes principais (a constante cosmológica $\Lambda$ e a matéria escura fria, "Cold Dark Matter").

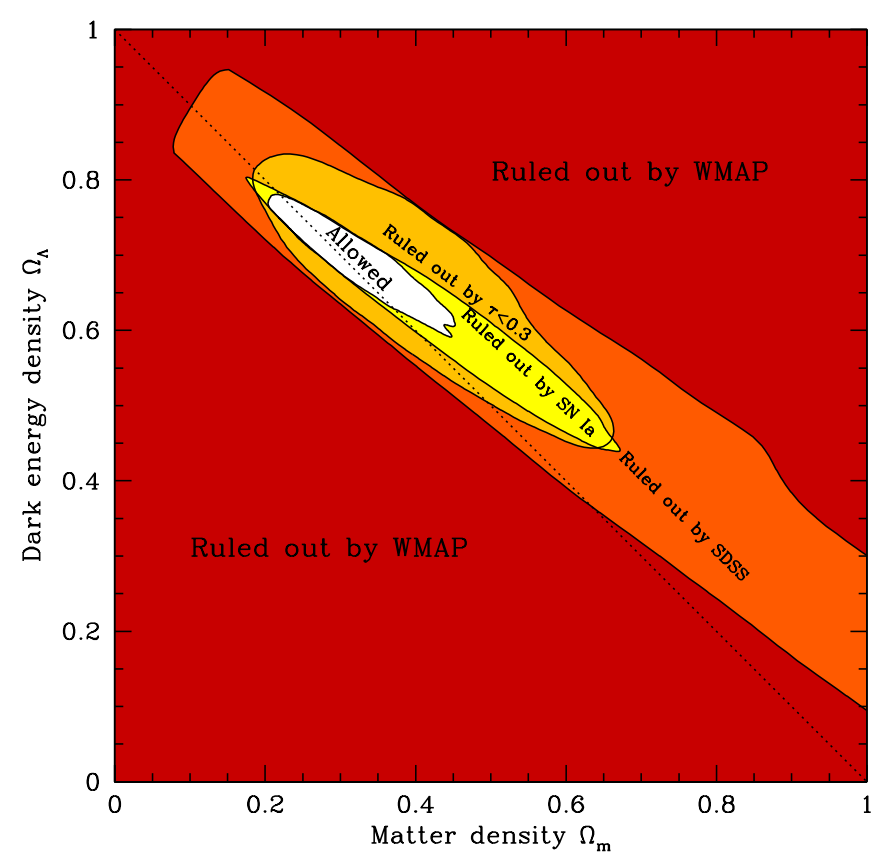

Figura 2.3: Valores permitidos para $\Omega_{m} \equiv \Omega_{D M 0}+\Omega_{B 0}$ e $\Omega_{\Lambda}$, extraído de [?]. Os vínculos dizem respeito a dados dos experimentos Sloan Digital Sky Survey (SDSS) e WMAP, além de restrições provenientes de observações de supernovas. 


\subsubsection{Idade do universo}

Um outro teste importante para o MCP consiste na previsão da idade do universo desde o Big-Bang. Utilizando (2.1) , (2.10) e (2.13), podemos escrever:

$$
\begin{aligned}
\left(\frac{\dot{a}}{a}\right)^{2}=H_{0}^{2}\left[\Omega_{D M 0+B 0}(1+z)^{3}+\Omega_{\Lambda 0} f(z)+\Omega_{R 0}(1+z)^{4}\right. & \left.+\Omega_{K 0}(1+z)^{2}\right] \\
& =H_{0}{ }^{2} E(z)^{2},
\end{aligned}
$$

que define a função $E(z)$ e onde foi utilizada uma função $f(z)$ para genericamente expressar uma possível dependência da densidade de energia escura com o redshift. No caso em que a energia escura é a constante cosmológica, $\Lambda$, devemos ter $f(z)=1$.

Utilizando (2.24), podemos calcular o tempo de expansão do universo desde seu passado remoto até o redshift $\mathrm{z}$ utilizando:

$$
t(z)=\int \frac{d a}{\dot{a}}=H_{0}^{-1} \int_{z}^{\infty} \frac{d z}{(1+z) E(z)} .
$$

Para redshifts moderadamente altos, da ordem de 1, onde o termo de matéria não-relativística domina, a equação (2.25) devolve:

$$
t(z) \simeq \frac{2}{3 H_{0} \Omega_{D M 0+B 0}^{1 / 2}}(1+z)^{-\frac{3}{2}},
$$

colocando por exemplo a datação de idade de estrelas velhas também como uma interessante medida da quantidade de matéria no universo. Um modelo que prediga um universo muito mais novo do que por exemplo o tempo de vida de estrelas velhas ([?] prevê idades da ordem de $11.10^{9}$ anos) deve ser imediatamente descartado. Por exemplo, dados do WMAP para o modelo $\Lambda C D M$ prevêem um universo com idade de $t_{0}=(13.7 \pm 0.2) 10^{9}$ anos [?].

\subsection{Distâncias em Cosmologia}

Como já poderia ser esperado, as medidas de distância em cosmologia não podem ser exatamente chamadas de triviais. O fato do fator de escala variar com o tempo acaba colocando algumas dificuldades no processo, o que motiva algumas definições diferentes, cada uma delas mais conveniente em determinada situação do que em outra, apenas com a característica comum de que todas elas são derivadas da distância medida em um referencial comóvel.

Uma distância comóvel que merece particular atenção é aquela percorrida pela luz, por exemplo desde um instante $t=0$. Em um intervalo $d t$, a luz percorre uma distância comóvel dada por $d \eta=\frac{d t}{a}(\mathrm{c}=1)$, o que resulta: 


$$
\eta \equiv \int_{0}^{t} \frac{d t^{\prime}}{a\left(t^{\prime}\right)}
$$

Ou seja, a importância de $\eta$ está no fato de que nenhuma informação pode ter se propagado mais longe do que isso desde o começo do tempo, ou seja, regiões separadas por uma distância (comóvel) maior do que $\eta$ não podem ter uma conexão causal.

Outra distância comóvel importante seria aquela medida entre um emissor e um observador. Conforme já apontado em (2.8), para um objeto situado em um redshift $z=\frac{1}{a-1}$, teremos:

$$
\chi(a)=\int_{t_{1(a)}}^{t_{0}} \frac{d t^{\prime}}{a\left(t^{\prime}\right)}=\int_{a}^{1} \frac{d a^{\prime}}{a^{\prime 2} H\left(a^{\prime}\right)} .
$$

A expressão (2.26) por sua vez pode ser utilizada para uma outra medida de distância bastante utilizada em astronomia, a chamada distância angular. Ela consiste na medida do ângulo $\theta$ que subentende um objeto de tamanho físico $l$. Para um ângulo pequeno, a distância é dada por:

$$
d_{A}=\frac{l}{\theta} .
$$

Em um universo plano ${ }^{8}$, levando em consideração as medidas feitas por um observador comóvel, teremos $\theta=\frac{l(a) / a}{\chi(a)}$, o que implica, depois de inserida em $(2.27)$ :

$$
d_{A}^{\text {plano }}=a \chi=\frac{\chi}{1+z} .
$$

Além dessas distâncias, outra que aparece com relativa freqüência é a chamada distância luminosidade, que surge num contexto de medidas de fluxos (potência por unidade de área) de objetos com luminosidade (potência) conhecida. A idéia é relativamente simples. Um observador em um referencial comóvel deve medir um fluxo de uma fonte (localizada na origem) com luminosidade L e a uma distância $\chi$ :

$$
F=\frac{L(\chi)}{4 \pi \chi^{2}}
$$

Entretanto, a energia por unidade de tempo atravessando uma camada esférica situada a uma distância $\chi(a)$ da fonte será reduzida por um fator de $a^{2}$, já que, além da redução por um fator de a do número de fótons cruzando essa camada esférica por unidade de tempo, também haverá uma diminuição da energia de cada fóton, ambas resultado da expansão do universo. Isso nos permite escrever:

\footnotetext{
${ }^{8}$ As relações para universos com curvaturas não nulas dependem da densidade de energia dos mesmos e podem, por exemplo ser encontradas em [?].
} 


$$
F=\frac{L a^{2}}{4 \pi \chi^{2}}=\frac{L}{4 \pi d_{l}^{2}}
$$

a última igualdade definindo a distância luminosidade $d_{l}$ como sendo $\frac{\chi}{a}$, o que nos permite escrever, utilizando (2.10) e (2.26):

$$
d_{l}(z)=(1+z) \chi(z)=(1+z) \int_{0}^{z} \frac{d z^{\prime}}{H\left(z^{\prime}\right)}
$$

o que fornece, depois de utilizar (2.24):

$$
\begin{aligned}
& d_{l}(z)=(1+z) \int_{0}^{z} \frac{d z^{\prime}}{H\left(z^{\prime}\right)}= \\
& \int_{0}^{z} \frac{\frac{(1+z)}{H_{0}} d z^{\prime}}{\sqrt{\Omega_{M 0}(1+z)^{3}+\Omega_{R 0}(1+z)^{4}+\Omega_{K 0}(1+z)^{2}+\frac{\rho(z)}{\rho_{0}}\left(1-\Omega_{M 0}-\Omega_{R 0}-\Omega_{K 0}\right)}}
\end{aligned}
$$

$\operatorname{com} \Omega_{M 0} \equiv \Omega_{D M 0+B 0}$ e $\rho(z)$ correspondendo à densidade de energia da energia escura. As medidas de distância luminosidade e distância angular, por dependerem do conteúdo energético do universo, aparecem também como importantes fontes de vínculos astronômicos [?]. Por exemplo, as derivadas de (2.30) permitem obter conexões diretas com observáveis físicos, como a curvatura do universo [?, ?].

\subsection{Nucleossíntese primordial e a radiação cós- mica de fundo}

O modelo do Big Bang descreve a evolução do universo a partir de um estado de extrema temperatura e pressão. Naquela época, a pressão da radiação era uma importante componente do conteúdo energético do universo. À medida que o universo se expandiu e esfriou, a matéria até então em estado de um plasma de partículas e radiação eletromagnética se condensou para muito posteriormente formar as galáxias que observamos hoje.

O conteúdo de matéria e radiação do universo varia bastante dependendo da época considerada. Estruturas atômicas têm geralmente energias de ligação da ordem de algumas dezenas de $\mathrm{eV}$ enquanto as nucleares ficam na casa de $\mathrm{MeV}$. Dessa forma, quando a temperatura do universo era maior do que esses valores, essas estruturas não podiam existir como estados ligados. Por outro lado, quando a temperatura do universo era muito maior do que a energia de repouso de uma determinada partícula, a energia dos fótons da radiação era suficiente para produzir essa partícula (e sua respectiva antipartícula) em grandes quantidades. Sendo assim, a temperatura de uma determinada época do universo primordial 
determina qual tipo de partícula elementar será predominante em determinada época.

Para obtermos resultados quantitativos, é essencial agora sabermos qual a função de distribuição $f_{i}(\mathbf{p}, t)$ dessas partículas (onde i é um índice que dá conta das várias espécies, t é o tempo e p o momento. A dependência espacial é descartada por argumentos de homogeneidade).

As diferentes espécies de partículas interagem constantemente, trocando energia e momento. Se a taxa $\Gamma$ dessas reações for muito maior que a taxa de expansão do universo $H(t)=\frac{\dot{a}}{a}$, pode-se estabelecer uma condição de equilíbrio térmico a uma temperatura $T$ entre as partículas interagentes. Nessas condições, as partículas podem ser tratadas como um gás ideal de bósons ou férmions, com função de distribuição dada por:

$$
f_{i}(\mathbf{p}, t) d^{3} \mathbf{p}=\frac{g_{i}}{(2 \pi)^{3}} \exp \left[\left(E(p)-\mu_{i}\right) / T_{i}(t) \pm 1\right]^{-1} d^{3} \mathbf{p},
$$

onde $g_{i}$ é um fator de degenerescência devido ao spin, $E(p)=\sqrt{p^{2}+m^{2}}$ e $\mu_{i}$ é o potencial químico (sinal + para férmions, - para bósons). O que pode ocorrer agora é que, no decorrer da evolução cósmica, a taxa de reação $\Gamma(t)$ para alguma das espécies $i$ pode ficar abaixo de $H(t)$. Nesse caso, essa espécie se desacopla do restante e evolui de maneira independente. Para um caso simples, em que esse desacoplamento ocorre instantaneamente em $t=t_{D}$, para $t>t_{D}$ teremos:

$$
f_{\text {dec }}=f_{i}\left(p \frac{a(t)}{a\left(t_{D}\right)}, t_{D}\right),
$$

o fator $p \frac{a(t)}{a\left(t_{D}\right)}$ sendo proveniente do redshift no momento p.

Em alguns limites de interesse prático, podemos calcular de maneira aproximada alguns observáveis físicos a partir de (2.31). Temos:

$$
\begin{gathered}
n=\int f(\mathbf{p}) d^{3} \mathbf{p}=\frac{g}{2 \pi^{2}} \int_{m}^{\infty} \frac{E\left(E^{2}-m^{2}\right)^{\frac{1}{2}} d E}{\exp \left(\frac{E(p)-\mu}{T}\right) \pm 1}, \\
\rho=\int E f(\mathbf{p}) d^{3} \mathbf{p}=\frac{g}{2 \pi^{2}} \int_{m}^{\infty} \frac{E^{2}\left(E^{2}-m^{2}\right)^{\frac{1}{2}} d E}{\exp \left(\frac{E(p)-\mu}{T}\right) \pm 1} .
\end{gathered}
$$

onde $n$ é a densidade de número das partículas relativísticas e $\rho$ é a densidade de energia.

Quando as partículas são altamente relativísticas $(T \gg m)$ e não-degeneradas $(T \gg \mu)$ obtemos:

$$
n \approx \frac{g}{2 \pi^{2}} \int_{0}^{\infty} \frac{E^{2} d E}{e^{\frac{E}{T}} \pm 1}=\left\{\begin{array}{cc}
\frac{3}{4} \frac{\zeta(3)}{\pi^{2}} g_{f} T^{3} & \text { férmions } \\
\frac{\zeta(3)}{\pi^{2}} g_{b} T^{3} & \text { bósons }
\end{array}\right.
$$




$$
\rho \approx \frac{g}{2 \pi^{2}} \int_{0}^{\infty} \frac{E^{3} d E}{e^{\frac{E}{T}} \pm 1}=\left\{\begin{array}{cc}
\frac{7}{8} g_{f} \frac{\pi^{2}}{30} T^{4} & \text { férmions } \\
g_{b} \frac{\pi^{2}}{30} T^{4} & \text { bósons }
\end{array}\right.
$$

onde $\zeta(3) \approx 1.202$ é a função zeta de Riemman de ordem 3 .

Apenas como exemplo, podemos calcular qual a densidade de energia prevista para a radiação cósmica de fundo no instante atual. Temos:

$$
\rho_{\gamma} \simeq g_{\gamma} \frac{\pi^{2}}{30} T^{4} \simeq 2.10^{-51}(G e V)^{4}
$$

onde se utilizou $T=2,7 \mathrm{~K}$ e $g_{\gamma}=2$ (dois estados de polarização para o fóton).

Resultados para várias espécies de partículas podem ser conseguidos de maneira análoga, utilizando aproximações convenientes. Os cálculos são bastante extensos e existe uma vasta literatura na área para os interessados [?].

\subsubsection{Era da radiação}

Ela ocorreu por volta de alguns segundos ${ }^{9}$ após o Big-Bang e se estendeu até um redhift $z$ da ordem de 3500. Foi nessa fase que os primeiros núcleos atômicos começaram a se formar, em um processo conhecido como nucleossíntese. Prótons e nêutrons remanescentes dos primeiros instantes posteriores ao Big-Bang se agrupam para formar os primeiros núcleos estáveis.

Quando prótons e nêutrons tornam-se não-relativísticos, sua densidade pode ser expressa pela distribuição de Maxwell-Boltzmann:

$$
n=n_{\text {spin }} \frac{T^{\frac{3}{2}}}{(2 \pi)^{\frac{3}{2}}} e^{-\frac{E}{T}},
$$

onde $n_{\text {spin }}$ é o número de estados de spin diferentes. De (2.36) podemos calcular a relação existente entre o número de prótons e nêutrons para uma dada temperatura $\mathrm{T}$ :

$$
\frac{n_{n}}{n_{p}}=\left(\frac{m_{n}}{m}\right)^{\frac{3}{2}} e^{-\frac{m_{n}-m_{p}}{T}}
$$

sendo $m_{n}-m_{p} \approx 1,3 \mathrm{MeV}$.

O núcleo mais importante formado nesta época é o do deutério ${ }^{2} \mathrm{H}$, com energia de ligação podendo ser estimada pela seguinte reação:

$$
m_{n}+m_{p}-m_{D}=2,22 \mathrm{MeV}
$$

onde $m_{n}$ é a massa do nêutron, $m_{p}$ a do próton e $m_{D}$ a do deutério.

\footnotetext{
${ }^{9} \mathrm{O}$ período anterior a esse envolve escalas de energia maiores que $1 \mathrm{TeV}$ onde a compreensão das interações entre partículas ainda não é completa, de modo que não será abordado neste texto.
} 
A partir desse, núcleos estáveis de hélio $\left({ }^{4} \mathrm{He}\right.$ e $\left.{ }^{3} \mathrm{He}\right)$, assim como berílio $\left({ }^{7} \mathrm{Be}\right)$ e lítio $\left({ }^{7} \mathrm{Li}\right)$, começam a aparecer via reações de fusão nuclear. Essa produção desses elementos recebe o nome de nucleossíntese e observações da composição química de estrelas tendem a dar amplo respaldo para isso [?].

\subsubsection{Era da matéria}

Por volta de um redshift $\mathrm{z}=3233_{-210}^{+194}[?], \mathrm{T} \approx 10^{4} \mathrm{~K}$, já que

$$
T \approx T_{0}(1+z)=2.73(1+z)
$$

acontece a igualdade da densidade de energia da matéria e da radiação. A partir daí, a matéria passa a dominar e a temperatura do universo transita de uma dependência do tipo $\mathrm{T} \propto t^{-\frac{1}{2}}$ para $\mathrm{T} \propto t^{-\frac{2}{3}}$.

Quando a temperatura do universo decai para cerca de 3000K, os primeiros átomos neutros estáveis se formam e matéria e radiação começam a deixar de ser acoplados. Nessa temperatura, os fótons já não têm tanta energia e os elétrons se tornam suficientemente lentos, a ponto de serem capturados pelos núcleos atômicos, em um processo que recebe o nome de recombinação. Um dos átomos formados nessa época é o do elemento mais abundante no universo hoje, o hidrogênio. Seria natural supor que a formação do hidrogênio se dá quando a temperatura da radiação é algo em torno de $13,6 \mathrm{eV}$, valor da sua energia de ligação. Porém, a ligação acaba ocorrendo para uma temperatura da ordem de 3 $\mathrm{eV}$, porque a energia liberada na ligação reaquece os elétrons.

Até essa temperatura, os fótons ainda interagiam com os elétrons via espalhamento Thomson e o universo era completamente opaco à radiação eletromagnética. No decorrer do processo de captura dos elétrons, os fótons começam a deixar de ser espalhados (já que os átomos formados são neutros), dando início a uma fase que marca o desacoplamento entre matéria e radiação. Estes fótons, agora livres, permeiam o universo e são a fonte do que hoje dizemos ser a radiação cósmica de fundo (RCF). A chamada última superfície de espalhamento, situada onde supostamente aconteceram as últimas interações destes fótons antes de eles ficarem livres acontece para um $z$ médio da ordem de $10^{3}$ (1089 \pm 1 , de acordo com [?]). Na realidade, esse valor não é exato, porque essa região acaba tendo uma determinada espessura $(195 \pm 2)$ [?], já que alguns fótons se desacoplam antes dos outros.

\subsubsection{Radiação cósmica de fundo}

Nosso universo, hoje, é preenchido por essa radiação que escapou do acoplamento com os bárions depois de passar pela última superfície de espalhamento. A expansão do universo fez com que esses fótons tivessem sua energia diminuída, já 
que tiveram seu comprimento de onda esticado. Os fótons que escaparam estavam em equilíbrio térmico, o que implica em uma distribuição do tipo corpo negro, de Planck (2.31), que, em unidades convencionais, fornece:

$$
n_{\gamma}(\nu) d \nu=\frac{8 \pi h \nu^{3} d \nu}{c^{3}\left(e^{\frac{h \nu}{T}}-1\right)},
$$

onde $n_{\nu}$ é a densidade de número dos fótons com freqüência $\nu$. Não acontecendo aniquilação ou criação de partículas, deve haver conservação do número de fótons e, sendo (2.38) válida em um instante $t$, ela deve continuar válida para os instantes seguintes.

Em 1948, George Gamow, Ralph Alpher e Robert Herman tiveram a idéia de calcular qual seria a temperatura desse espectro primordial, suposto de corpo negro, proveniente desses fótons da última superfície de espalhamento. Seus resultados apontaram para uma temperatura T da ordem de 5K. Anos depois, Penzias e Wilson (1965) identificaram de fato a existência dessa radiação e, atualmente, as informações provenientes do seu espectro [?, ?], bem como a descoberta das suas anisotropias é um dos maiores triunfos da cosmologia moderna (dados do instrumento FIRAS, a bordo do satélite Cobe, lançado em 1989, apontam para um valor $\left.\mathrm{T}_{\gamma}=2,728 \pm 0,002 \mathrm{~K}[?]\right)$.

Uma das importantes informações que pode ser extraída de dados da radiação cósmica de fundo diz respeito às perturbações de densidade no universo primordial. Durante o período entre a igualdade de densidade de matéria e radiação e a recombinação, apenas perturbações na matéria escura não-bariônica poderiam crescer, porque os bárions eram impedidos de se aglomerar pela pressão da radiação. Uma vez que a recombinação acontece, os bárions podem cair nos poços de potencial da matéria escura. Dessa maneira, a presença de matéria escura acaba por permitir que o crescimento de estruturas aconteça mais cedo do que ocorreria para um universo em que ela não existisse.

\subsection{Resumo}

Para fechar o capítulo, um resumo dos principais pontos apresentados:

- O universo é dominado (cerca de 95\% do total energético) por componentes escuras, de natureza ainda não bem determinada;

- O universo apresenta uma seção espacial aproximadamente plana, resultado de acordo com previsões da teoria inflacionária;

- A radiação cósmica de fundo, a lei de Hubble e os dados da nucleossíntese primordial, como por exemplo abundâncias de elementos leves, são consistentes com o modelo cosmológico padrão. 
Neste tópico encerra-se a discussão sobre o modelo cosmológico padrão. O capítulo seguinte adotará uma postura diferente, aprofundando-se em apenas um aspecto aqui apresentado e que será o foco da dissertação daqui pra frente. Discutiremos o papel da matéria escura no cenário cosmológico atual, em que contexto ela surge, as primeiras observações feitas, além de algumas formas de modelagem, dando particular enfoque aos modelos numéricos. Ao leitor interessado em se aprofundar em outros aspectos do MCP, é sugerida a leitura das referências $[?, ?, ?, ?]$. 


\section{Capítulo 3}

\section{Matéria escura}

O paradigma cosmológico atual afirma que uma parcela significativa do conteúdo de matéria do universo deve se encontrar na forma de partículas da chamada matéria escura fria não-bariônica cuja natureza desconhecida tem desafiado legiões de cosmólogos.

As primeiras especulações a respeito da existência da matéria escura remontam aos trabalhos do astrônomo suiço Fritz Zwicky [?] que, por volta de 1933, observou que as velocidades de algumas galáxias em aglomerados eram de fato muito maiores do que o que era previsto pelas relações de massa-luminosidade [?]. Nessa época, a lei de Hubble relacionando distâncias e velocidades já era conhecida (embora a constante de Hubble utilizada tivesse um valor incorreto) e já estavam disponíveis curvas de rotação para diversas galáxias espirais, o que permitia obter estimativas, ainda que grosseiras, das razões entre massa e luminosidade nas partes centrais dessas galáxias.

O trabalho de Zwicky se baseou então na medida de velocidades radiais de sete galáxias pertencentes ao chamado aglomerado de Coma. Suas observações constataram um fato curioso, o de que as galáxias individuais tinham velocidades radiais que diferiam da velocidade média do aglomerado, com uma dispersão RMS de cerca de $700 \mathrm{~km}_{.} \mathrm{s}^{-1}$. Essa dispersão foi interpretada por ele na época como uma medida da energia cinética por unidade de massa das galáxias ali presentes. Fazendo em seguida uma estimativa do raio do aglomerado, ele pôde inferir a massa total do mesmo utilizando o teorema de Virial [?], que permite estabelecer uma relação simples e direta entre as energias cinética $(\mathrm{K})$ e potencial $(\mathrm{W})$ de um sistema isolado:

$$
\frac{d^{2} I}{d t^{2}}=2 K+W
$$

onde I é o momento de inércia do sistema.

Posteriormente, da comparação das razões massa-luminosidade $\left(\frac{M}{L}\right)$ obtidas pelo seu método com aquelas resultantes do estudo de curvas de rotação, ele 
percebeu ter alcançado um resultado para $\frac{M}{L}$ que era cerca de 400 vezes maior ${ }^{1}$, fato que o levou a concluir que a maior parte da massa do aglomerado se encontrava na forma de uma matéria invisível ou escura ("dark"). Ou seja, ele cunhou um termo para designar um tipo de matéria que não é detectada por seus efeitos eletromagnéticos ou de qualquer outra interação que não seja a gravitacional.

Algumas décadas depois, outros trabalhos [?, ?, ?] passaram a sugerir a existência de grandes quantidades de matéria escura não apenas em aglomerados mas também ao redor de galáxias isoladas, em uma região chamada por eles de "halo". Esses halos deveriam se estender até distâncias muito maiores do que aquela até onde se poderia encontrar matéria luminosa e supostamente conteriam a maior parte da massa total da galáxia. Atualmente, a idéia mais aceita é a de que esse halos contém matéria da mesma natureza da que foi encontrada por Zwicky, de forma que também adotaremos essa postura nesta dissertação. Entenderemos por halos regiões onde podemos encontrar matéria escura, seja ela em escalas galácticas (ordem de kpc) ou de aglomerados (ordem de Mpc).

\subsection{Evidências da necessidade da existência de matéria escura}

Como discutido no Cap. 2, a matéria escura é um ingrediente essencial do modelo $\Lambda C D M$. Entretanto, além dos seus já citados efeitos observados em curvas de rotação de galáxias e aglomerados, hoje se sabe que existem outras situações em que ela se faz necessária, o que é por sua vez interessante por se mostrar como uma espécie de teste de consistência da teoria. Uma dessas situações diz respeito à formação de estruturas.

Hoje sabemos que, na ausência de matéria escura, o universo teria permanecido uniforme por um tempo bem maior antes de formar galáxias, estrelas ou planetas. Sendo a força newtoniana a única interação de longo alcance conhecida capaz de responder pelo movimento de matéria em escalas tão grandes e pela impossibilidade de um meio completamente uniforme lançar as sementes para as irregularidades capazes de produzir atração gravitacional, a suposição natural é de que todas as grandes estruturas observadas hoje devem ter sido geradas por pequenas flutuações de densidade ocorridas em certas regiões do universo pouco tempo depois do Big-Bang. Tais flutuações deveriam ficar "impressas" na RCF, deixando uma espécie de assinatura bem característica a ser procurada. Atualmente, graças aos dados de experimentos como o WMAP, é sabido que a matéria ordinária não seria capaz de responder sozinha pela formação das estruturas observadas hoje sem deixar um sinal na RCF maior do que o observado [?], já que o acoplamento que existiu entre bárions e radiação durante um relativamente

\footnotetext{
${ }^{1}$ Nesse aglomerado, as estimativas atuais apontam para uma razão $\frac{M}{L}$ que é cerca de 30 vezes maior do que a razão $\frac{M}{L}$ obtida para as partes luminosas das galáxias típicas.
} 
longo período posterior ao Big-Bang impediria a aglomeração ("clustering") até bem depois da época da igualdade entre matéria e radiação.

Por outro lado, a matéria escura, que não deve interagir com fótons, seria capaz de permitir pequenas flutuações de densidade (consistentes com dados da $\mathrm{RCF}$, que prevêem flutuações bem pequenas de temperatura do espectro da RCF, da ordem de uma parte em $10^{5}$ ) crescendo por longos intervalos de tempo antes de a matéria (bariônica) e a radiação se desacoplarem, o que poderia fornecer um cenário mais atraente para a formação de estruturas.

Esse tipo de abordagem pode ser compreendido da seguinte maneira: a distribuição de matéria no universo pode, com razoável aproximação, ser descrita por um fluido viscoso, não estático. Esse fluido, pelo efeito da competição entre a pressão da radiação e a força gravitacional, acaba por apresentar regiões de maior compressão ou rarefação, o que, por sua vez, se reflete em flutuações aleatórias de densidade em torno de uma média, $\bar{\rho}(t)$. Definimos então o chamado contraste de densidade, $\delta$ por:

$$
\delta(\mathbf{r}, t)=\frac{\rho(\mathbf{r}, t)-\bar{\rho}(t)}{\bar{\rho}(t)},
$$

onde $\rho$ é a densidade, função de uma coordenada comóvel $\mathrm{r}$ e do tempo t.

Decompondo esse padrão arbitrário de flutuações (3.2) em termos de uma série de ondas independentes, cada uma com um comprimento de onda $\lambda_{k}$ e uma amplitude $\delta_{k}$, ou seja, expandindo em uma série de Fourier, teremos:

$$
\delta(\mathbf{r}, t) \propto \sum \delta_{k}(t) e^{i \mathbf{k} . \mathbf{r}}
$$

sendo $\mathbf{k}$ o vetor de onda.

Define-se então a chamada função de autocorrelação de massa por:

$$
\xi(r)=\left\langle\delta\left(\mathbf{r}_{\mathbf{1}}\right) \delta\left(\mathbf{r}+\mathbf{r}_{\mathbf{1}}\right)\right\rangle \propto \sum\left\langle\left|\delta_{k}\right|^{2}\right\rangle e^{i \mathbf{k r}} .
$$

Por sua vez, os coeficientes $\left|\delta_{k}\right|^{2}$ definem o espectro de potência das flutuações de massa:

$$
P(k)=\left\langle\left|\delta_{k}\right|^{2}\right\rangle,
$$

ou seja, a função de autocorrelação de massa (3.4) é a transformada de Fourier do espectro de potência (3.5). É usual supor um espectro de potência do tipo lei de potência:

$$
P(k)=k^{n},
$$

onde $\mathrm{n}$ é o índice espectral. De acordo com os dados mais recentes do experimento WMAP, um cenário em que o espectro inicial de perturbações é independente de 
escala $^{2}$, o que implica em um índice espectral $n=1$, parece ser bastante viável, impulsionando ainda mais o MCP, já que medidas recentes apontam $n=0.99 \pm$ $0.04[?]$.

\subsection{Curvas de rotação}

Uma maneira relativamente simples de visualizar o problema das curvas de rotação de galáxias, consiste em analisar, do ponto de vista newtoniano, a seguinte situação: suponhamos um espaço preenchido por diferentes cascas esféricas concêntricas de raio $r^{\prime}$ e com densidade $\rho\left(r^{\prime}\right)$. Analisemos o potencial gerado por essas camadas. Separando as contribuições em termos para os quais $r^{\prime}<r$ e $r^{\prime}>r$, o potencial gravitacional gerado a uma distância $\mathrm{r}$ do centro pode ser calculado através de [?]:

$$
\Psi(r)=-4 \pi G\left[\frac{1}{r} \int_{0}^{r} \rho\left(r^{\prime}\right) r^{\prime 2} d r^{\prime}+\int_{r}^{\infty} \rho\left(r^{\prime}\right) r^{\prime} d r^{\prime}\right] .
$$

Logo, uma partícula-teste de massa unitária colocada nesse potencial, sofre a ação de uma força dada por:

$$
\mathbf{F}=-\nabla \Psi=-\frac{d \Psi(r)}{d r} \hat{\mathbf{e}}_{r}=-\frac{G M(r)}{r^{2}} \hat{\mathbf{e}}_{\mathbf{r}}
$$

onde $M(r)$ é a massa contida no interior uma esfera de raio $\mathrm{r}$ e $\hat{\mathbf{e}}_{\mathrm{r}}$ é um versor na direção r. De (3.8), podemos calcular qual deve ser a velocidade de uma partícula-teste percorrendo uma órbita circular de raio $r$ :

$$
v_{c}^{2}=r\left|\frac{d \Psi}{d r}\right|=r|\mathbf{F}|=\frac{G M(r)}{r} .
$$

A expressão (3.9) permite uma análise, a partir de velocidades circulares medidas, das possíveis distribuições de matéria responsáveis pelo movimento observado. No caso específico de galáxias (ou aglomerados), supondo que a massa $M$ contida dentro de um determinado raio $r$ pára de crescer a partir de um certo $r=r_{\text {max }}$, um resultado típico que poderia ser esperado para grandes distâncias $\left(r \gg r_{\max }\right)$ seria o seguinte (comportamento Kepleriano):

$$
v_{c} \propto r^{-\frac{1}{2}}
$$

Entretanto, diversas observações têm contrariado veementemente esse resultado [?, ?]. As curvas de rotação encontradas para diversas galáxias têm apresentado, para longas distâncias, um perfil dito plano, em que as velocidades de rotação de corpos situados a longas distâncias não decaem com o aumento de r.

\footnotetext{
${ }^{2} \mathrm{Um}$ resultado que é inclusive previsto pela teoria da inflação [?].
} 
$\mathrm{Na}$ realidade, o que parece ocorrer é uma situação em que as velocidades circulares se tornam aproximadamente constantes a partir de um determinado raio, ou seja, teríamos uma distribuição de massa que cresceria com $\mathrm{r}(M(r) \propto r)$ para grandes distâncias:

$$
v_{c}=\sqrt{\frac{G M(r)}{r}} \propto c,
$$

onde c é uma constante. Nesse caso, o potencial newtoniano é dado por:

$$
\Psi(r)=v_{c}^{2} \ln \left(\frac{r}{r_{f}}\right),
$$

com $r_{f}$ sendo uma distância de referência, para a qual $\Psi=0$. Utilizando a equação de Poisson [?], $\nabla^{\mathbf{2}} \Psi=4 \pi G \rho$, podemos encontrar a distribuição de massa responsável por esse comportamento:

$$
\rho(r)=\frac{v_{c}^{2}}{4 \pi G r^{2}} .
$$

A equação (3.11) expressa o chamado perfil isotérmico.

Esse tipo de comportamento é ainda constatado mesmo em regiões bem distantes do centro galáctico, onde não se observam mais estrelas luminosas (nem sempre observações no óptico são suficientes para colocar vínculos na quantidade de matéria escura nas galáxias [?]), graças à medidas de linhas de emissão de hidrogênio via radiotelescópios, estudos de galáxias satélites, efeitos de lentes gravitacionais fracas além de espectros de quasares. Essas medidas, por exemplo, prevêem halos de mais de $200 \mathrm{kpc}$, cerca de $230 \mathrm{kpc}$ para a Via-Láctea [?], com uma densidade média de matéria escura da ordem de $\rho_{d m} \approx 0.3 \mathrm{GeV} . \mathrm{cm}^{-3}$ (embora esse resultado dependa sensivelmente de como é modelado o halo). Na realidade, em alguns casos de galáxias satélites de galáxias espirais, já foram inclusive encontrados halos de mais de $400 \mathrm{kpc}$ [?]. 


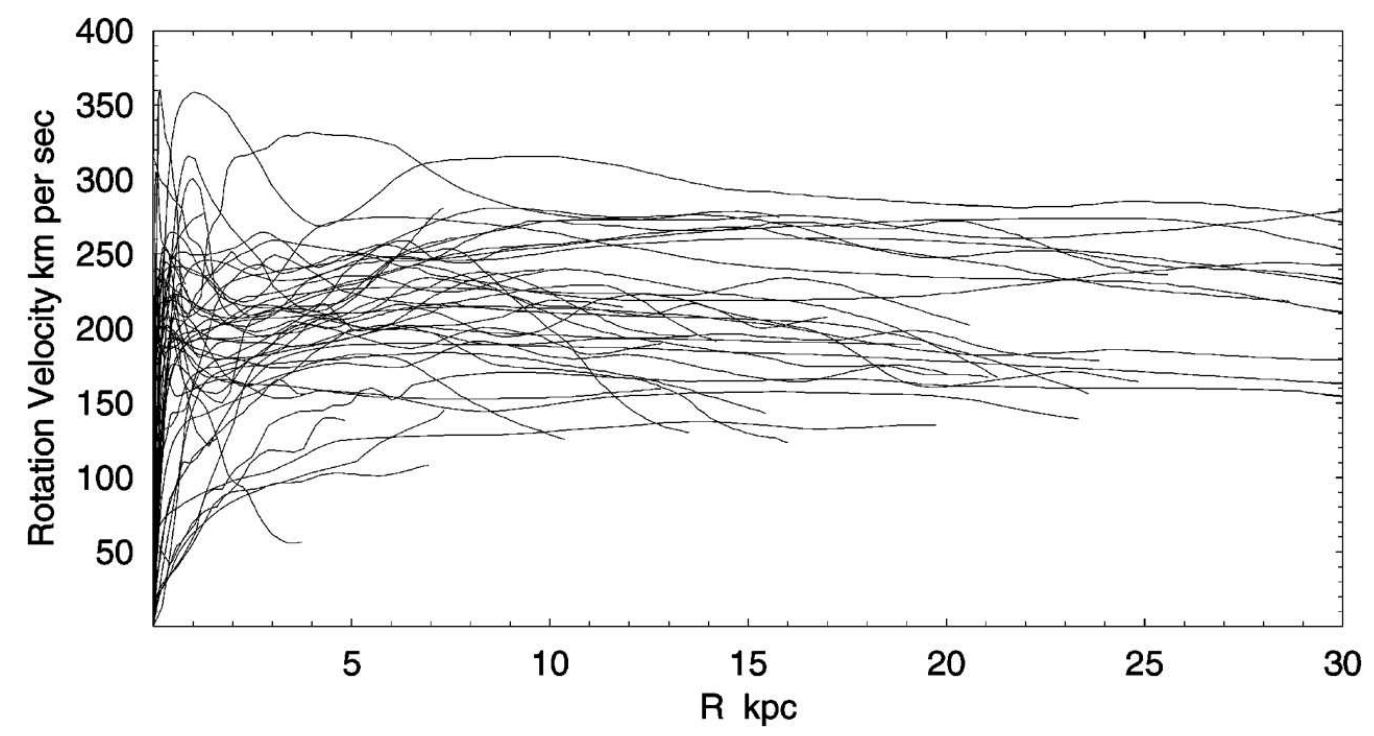

Figura 3.1: Extraído da referência [?], apresenta mais de 100 curvas de rotação diferentes, sugerindo fortemente um perfil plano para longas distâncias.

\subsubsection{Um pouco sobre a equação acolisional de Boltzmann}

Outras informações que podem ser extraídas de medidas de velocidades em galáxias e aglomerados se relacionam com a teoria da matéria escura fria por intermédio da chamada equação acolisional de Boltzmann ${ }^{3}$, que descreve o movimento no espaço de fases de um fluido de partículas que não colidem, encaixe perfeito para o modelo CDM.

A equação de Boltzmann pode ser vista da seguinte maneira: imaginemos um fluido de partículas que não colidem entre si, se movendo sob ação de um potencial $\Psi(\mathbf{x}, t)$. Se soubermos a função de distribuição do sistema $f(\mathbf{x}, t)$ no espaço de fases em algum instante $t=t_{0}$, uma descrição completa do sistema nos instantes futuros pode ser dada, já que $f(\mathbf{x}, t)$ obedece uma equação análoga à equação da continuidade para um fluido ordinário:

$$
\frac{\partial f}{\partial t}+\sum_{\alpha=1}^{6} \frac{\partial\left(f \dot{\omega}_{\alpha}\right)}{\partial \omega_{\alpha}}=\frac{\partial f}{\partial t}+\mathbf{v} \cdot \nabla f-\nabla \Psi \cdot \frac{\partial f}{\partial \mathbf{v}}=0,
$$

onde $\omega \equiv(\mathbf{x}, \mathbf{v})$ e $\dot{\omega} \equiv(\dot{\mathbf{x}}, \dot{\mathbf{v}})=(\mathbf{v},-\nabla \Psi)$.

Com um pouco de manipulação algébrica [?], pode-se obter, a partir de (3.12) as chamadas equações de Jeans, que são momentos das equações de Boltzmann:

\footnotetext{
${ }^{3}$ Como estamos lidando com matéria escura em halos, apresentaremos apenas a versão não relativística da mesma, sem correções devido à expansão de Hubble. Maiores informações podem ser encontradas em [?].
} 


$$
\rho \frac{\partial \overline{v_{j}}}{\partial t}+\rho \overline{v_{i}} \frac{\partial \overline{v_{j}}}{\partial x_{i}}=-\rho \frac{\partial \Psi}{\partial x_{j}}-\frac{\partial\left(\rho \sigma_{i j}^{2}\right)}{\partial x_{i}},
$$

onde $\left(x_{i}, v_{j}\right)$ são as posições e velocidades em alguma base ortonormal e $\sigma_{i j}{ }^{2}$ é a dispersão de velocidades, dada por $\sigma_{i j}^{2}=\overline{v_{i} v_{j}}-\overline{v_{i}} \overline{v_{j}}$.

Para o caso dos halos, é usual a busca de alguma forma de simetria buscando a simplificação do problema. Aproveitando apenas o lado esquerdo de (3.12), podemos reescrever a equação de Boltzmann explicitamente em coordenadas esféricas $(r, \theta, \phi)$ :

$$
\begin{aligned}
\frac{\partial f}{\partial t}+ & v_{r} \frac{\partial f}{\partial r}+\frac{v_{\theta}}{r} \frac{\partial f}{\partial \theta}+\frac{v_{\phi}}{r \sin \theta} \frac{\partial f}{\partial \phi}-\frac{1}{r}\left[v_{\phi}\left(v_{r}+v_{\theta} \cot \theta\right)+\frac{1}{\sin \theta} \frac{\partial \Psi}{\partial \phi}\right] \frac{\partial f}{\partial v_{\phi}} \\
+ & \frac{1}{r}\left(v_{{ }_{\phi}}^{2} \cot \theta-v_{r} v_{\theta}-\frac{\partial \Psi}{\partial \theta}\right) \frac{\partial f}{\partial v_{\theta}}+\left(\frac{v_{\theta}^{2}+v_{\phi}^{2}}{r}-\frac{\partial \Psi}{\partial r}\right) \frac{\partial f}{\partial v_{r}}=0 .
\end{aligned}
$$

Podemos executar o mesmo procedimento em (3.10). Desprezando velocidades do background e supondo a média das velocidades nula, obtem-se a seguinte equação para a dispersão radial de velocidades $\left(\sigma_{r}{ }^{2} \equiv\left\langle v_{r}^{2}\right\rangle-\left\langle v_{r}\right\rangle^{2}, v_{r}\right.$ sendo a velocidade na direção radial):

$$
\frac{1}{\rho} \frac{d}{d r}\left(\rho \sigma_{r}^{2}\right)+\frac{2 \beta \sigma_{r}^{2}}{r}=-\frac{G M(r)}{r^{2}}
$$

onde $\beta$ é o parâmetro de anisotropia, definido por $\beta \equiv 1-\frac{\sigma_{\theta}{ }^{2}}{\sigma_{r}{ }^{2}}$. Alguns trabalhos inclusive já propuseram que $\beta$ deveria seguir um perfil universal para estruturas formadas com matéria escura [?], mas isso parece não ser válido em geral [?].

\subsection{Perfis de densidade}

A estrutura de halos de matéria escura formados via colapso gravitacional provavelmente começou a receber uma maior atenção depois dos trabalhos de Gunn \& Gott [?] que, em 1972, mostraram que a estrutura virializada de halos poderia conter pistas para os parâmetros cosmológicos. Trabalhos analíticos posteriores, notadamente os baseados em modelos com condições iniciais independentes de escala, trouxeram novo impulso para a teoria, mostrando que os perfis dos halos poderiam ser bem aproximados por leis de potência e que a inclinação desses deveria depender sensivelmente dos parâmetros cosmológicos [?, ?].

Por outro lado, desde meados da década de 80, muitos trabalhos numéricos têm também tentado obter estimativas razoáveis para os possíveis perfis de densidade dos halos de matéria escura. Entretanto, grande parte dos primeiros resultados que surgiram na área [?, ?], influenciados pelos resultados analíticos supracitados, apresentavam uma tendência de tentar ajustar perfis do tipo lei de 
potência (notadamente $\rho \propto r^{-2}$, o chamado perfil isotérmico) às simulações de N-corpos.

Alguns anos depois, novos trabalhos surgiram [?, ?] e começaram a se mostrar capazes de encontrar as primeiras evidências de desvios de perfis do tipo lei de potência, graças ao sensível aumento da resolução das simulações. Além disso, outras propriedades de destaque foram descobertas, uma delas sendo a aparente universalidade que os halos (mais seguramente os de aglomerados) parecem apresentar [?], mesmo para intervalos de várias ordens de magnitude em massa ${ }^{4}$. Por essa universalidade entendemos que, indepentemente da massa, parâmetros cosmológicos e espectro de potência das flutuações iniciais de densidade, os perfis (supostos esfericamente simétricos) têm uma mesma dependência radial.

Ancorada nesses trabalhos, foi posteriormente levantada a possibilidade [?] (NFW) de que os halos pudessem ser descritos por uma função analítica simples, com apenas dois parâmetros (densidade e raio característicos, por exemplo). Essa função apresenta um perfil mais acentuado $\left(\rho \propto r^{-3}\right)$ do que o isotérmico para grandes distâncias porém mais "raso" $\left(\rho \propto r^{-1}\right)$, embora ainda divergente, próximo do centro. Dessa forma, o parâmetro

$$
\alpha=-\frac{d \log \rho(r)}{d \log r}
$$

varia suavemente entre 1 e 3 , sendo igual a 2 apenas no chamado raio característico $\left(r_{s}\right)$. Trabalhos recentes [?] inclusive apontam para a possibilidade de haver uma relação universal entre a inclinação radial para a densidade $\alpha$ e o parâmetro de anisotropia $\beta$.

Entretanto, embora outros trabalhos tenham obtido resultados similares aos encontrados no original de NFW, ainda é motivo de bastante controvérsia o valor exato de $\alpha$ para a porção mais interna do halo, bem como a existência ou não de uma dependência dele com a resolução da simulação. Artigos subseqüentes $[?$, ?, ?, ?] atestam para a existência de perfis ainda mais "rasos" próximos ao centro $\left(\alpha \propto r^{-1.5}\right)$ e outros, como [?, ?], afirmam não ser possível concluir que os halos possuem de fato perfil universal, suas características poderiam depender de fatores tais como a massa e a história de formação do halo.

Para aumentar ainda mais a indefinição, quase que paralelamente, começou-se a trabalhar com outras possibilidades [?], por exemplo a de que os halos talvez apresentassem na porção mais interna não um perfil divergente na origem, mas algo como um caroço duro de densidade constante. Outros, por exemplo [?], sugerem que são desvios da simetria esférica que poderiam resolver o problema, invocando a necessidade de as simulações incorporarem possíveis efeitos da triaxialidade dos halos.

Atualmente, uma das maneiras para tentar selecionar entre os tipos de perfis consiste em estudos de galáxias com baixo brilho superficial (LSB). Galáxias dessa

\footnotetext{
${ }^{4}$ Embora isso ainda seja tema de muita discussão, como pode por exemplo ser visto em [?].
} 
natureza são fortemente dominadas por matéria escura, sendo suas populações estelares praticamente sem importância dinâmica. Com base apenas nesses dados, as comparações mais recentes parecem favorecer perfis do segundo tipo, com caroços de densidade constante próximos ao centro [?, ?]. Mesmo assim, as simulações numéricas com as maiores resoluções já feitas (cerca de $3.10^{15} M_{\odot}$ ) [?], ainda insistem em privilegiar perfis do tipo "cusp", com uma densidade na porção mais interna variando como uma lei de potência $\left(\rho \propto r^{-\gamma}\right)$, o que acaba por deixar a questão com uma resposta atual ainda inconclusiva.

Para aumentar a complexidade do problema, outros fatores têm sido apontados como relevantes para conclusões definitivas. Um deles, que aparece com força crescente é a suposta presença de buracos negros supermassivos no centro de muitas galáxias e cujos efeitos ainda não são completamente conhecidos [?, ?].

\subsubsection{Alguns perfis populares}

Apresentaremos a seguir, alguns perfis que aparecem de maneira recorrente na literatura e que se propõem a fornecer um bom ajuste para as densidades de matéria escura na vizinhança de galáxias e aglomerados. Os modelos em geral apresentam uma dependência radial para a densidade da forma:

$$
\rho(r)=\frac{\rho_{0}}{\left(\frac{r}{R}\right)^{\gamma}\left[1+\left(\frac{r}{R}\right)^{\alpha}\right]^{\left(\frac{\beta-\gamma}{\alpha}\right)}} .
$$

\subsubsection{Perfil N.F.W.}

O perfil radial proposto por NFW [?] tem a forma:

$$
\rho(r)=\frac{\rho_{s}}{\frac{r}{r_{s}}\left(1+\frac{r}{r_{s}}\right)^{2}},
$$

sendo $\rho_{s}$ e $r_{s}$ respectivamente a densidade característica e o raio característico do halo.

Integrando até um raio r, podemos obter a massa contida nesse halo:

$$
M(r)=\int_{0}^{r} 4 \pi r^{\prime 2} \rho\left(r^{\prime}\right) d r^{\prime}=4 \pi \rho_{s} r_{s} \int_{0}^{r} \frac{r^{\prime} d r^{\prime}}{\left(1+\frac{r^{\prime}}{r_{s}}\right)^{2}} .
$$

Tomando agora o chamado parâmetro de concentração $c \equiv \frac{r_{200}}{r_{s}}$, onde $r_{200}$ é o raio para o qual o halo teria uma massa 200 vezes maior que a massa de um halo formado por uma concentração de massa com a densidade crítica $\left(\rho_{c}=\right.$ $\left.277.3 h^{2} M_{\odot} k p c^{-3}\right)$, ou seja: $r_{200}=\left(\frac{M_{200}}{\frac{4}{3} \pi 200 \rho_{c r i t}}\right)^{1 / 3}$, e fazendo posteriormente a mudança de variável $y^{\prime}=\frac{r^{\prime}}{r_{200}}$ temos: 


$$
\begin{aligned}
M(r)=4 \pi \rho_{s} r^{3}{ }_{s} c^{2} \int_{0}^{y} \frac{y^{\prime} d y^{\prime}}{\left(1+c y^{\prime 2}\right)} & =4 \pi \rho_{s} r^{3}{ }_{s} c^{2}\left[-\frac{y}{c}(1+c y)^{-1}+\frac{1}{c^{2}} \ln (1+c y)\right] \\
& =4 \pi \rho_{s} r_{s}{ }_{s}\left[\ln \left(1+\frac{r}{r_{s}}\right)-\left(\frac{\frac{r}{r_{s}}}{1+\frac{r}{r_{s}}}\right)\right] .
\end{aligned}
$$

De (3.20), olhando para a massa em $r=r_{200}$, podemos concluir que :

$$
\rho_{s}=\frac{200 \rho_{c} c^{3}}{3}\left(\ln (1+c)-\frac{c}{1+c}\right) .
$$

Logo, podemos reescrever a densidade do halo em termos do parâmetro de concentração:

$$
\rho(y)=\frac{200 \rho_{c} c^{2}}{y(1+c y)^{2}}\left(\ln (1+c)-\frac{c}{1+c}\right)^{-1} .
$$

A inclinação logarítmica $\alpha(r)$ é dada por:

$$
\alpha(r)=1+2\left(\frac{c y}{1+c y}\right)=1+2\left(\frac{\frac{r}{r_{s}}}{1+\frac{r}{r_{s}}}\right) .
$$

Já o perfil circular de velocidades de partículas-teste orbitando esse halo é dado por (3.9):

$$
\begin{gathered}
v_{c}(r)=\left(\frac{G M(r)}{r}\right)^{\frac{1}{2}}=v_{200}\left(\frac{1}{y} \frac{\ln (1+c y)-\frac{c y}{1+c y}}{\ln (1+c y)-\frac{c}{1+c}}\right)^{\frac{1}{2}}= \\
v_{200}\left(\frac{r_{200}}{r} \frac{\ln \left(1+\frac{r}{r_{s}}\right)-\frac{\frac{r}{r_{s}}}{1+\frac{r}{r_{s}}}}{\ln \left(1+\frac{r}{r_{s}}\right)-\frac{r_{200}}{1+\frac{r_{s}}{r_{s}}}}\right)^{\frac{1}{2}},
\end{gathered}
$$

onde $v_{200}$ é a velocidade circular em $r_{200}$.

Para um caso em que o parâmetro de anisotropia $\beta$ em (3.15) é nulo, ou seja, na aproximação em que os movimentos aleatórios são isotrópicos, podemos ainda obter para a dispersão ${\sigma_{r}}^{2}$ no caso NFW:

$$
\begin{aligned}
\frac{d}{d r}\left(\rho{\sigma_{r}}^{2}\right)= & -\frac{G M(r)}{r^{2}} \rho(r) \Rightarrow \\
& \sigma_{r}^{2}(r)=-\frac{r}{r_{s}}\left(1+\frac{r}{r_{s}}\right)^{2} \int_{0}^{\frac{r}{r_{s}}} \frac{4 \pi G \rho_{s} r_{s}^{2}}{x^{3}(1+x)^{2}}\left[\ln (1+x)-\frac{x}{1+x}\right] d x
\end{aligned}
$$




\subsubsection{Perfil Moore et al.}

Baseado em uma série de simulações de alta resolução, [?] defendem uma forma ligeiramente diferente da proposta por NFW para o perfil dos halos escuros:

$$
\rho(r)=\frac{\rho_{m}}{\left(\frac{r}{r_{m}}\right)^{\frac{3}{2}}\left[1+\left(\frac{r}{r_{m}}\right)^{\frac{3}{2}}\right]} .
$$

A massa dentro de um raio $r$ é por sua vez:

$$
M(r)=\frac{8 \pi}{3} \rho_{m} r_{m}^{3}\left(\ln \left(1+(\widetilde{c} y)^{\frac{3}{2}}\right)\right)=\frac{8 \pi}{3} \rho_{m} r_{m}^{3} \ln \left(1+\left(\frac{r}{r_{m}}\right)^{\frac{3}{2}}\right),
$$

onde $\widetilde{c}=\frac{r_{200}}{r_{m}}$. A inclinação logarítmica $\alpha(r)$ nesse caso é dada por:

$$
\alpha(r)=\frac{3}{2}\left[1+\frac{\left(\frac{r}{r_{m}}\right)^{\frac{3}{2}}}{1+\left(\frac{r}{r_{m}}\right)^{\frac{3}{2}}}\right]
$$

tendo um valor assintótico de $\alpha=1.5$ próximo ao centro.

Por sua vez, o perfil de velocidades circulares nesse caso responde por (3.9):

$$
v_{c}(r)=v_{200}\left(\frac{1}{y} \frac{\ln \left(1+(\widetilde{c} y)^{\frac{3}{2}}\right)}{\ln \left(1+(\widetilde{c})^{\frac{3}{2}}\right)}\right)^{\frac{1}{2}}=v_{200}\left(\frac{r_{200}}{r} \frac{\ln \left(1+\left(\frac{r}{r_{m}}\right)^{\frac{3}{2}}\right)}{\ln \left(1+\left(\frac{r_{200}}{r_{m}}\right)^{\frac{3}{2}}\right)}\right)^{\frac{1}{2}}
$$

sendo $v_{200}$ novamente a velocidade em $r=r_{200}$.

No caso desse perfil, a equação de Jeans (3.15) com dispersão isotrópica de velocidades $(\beta=0)$ fornece:

$$
\sigma_{r}^{2}=-\frac{8 \pi G}{3}\left(\frac{r}{r_{m}}\right)^{\frac{3}{2}}\left(1+\left(\frac{r}{r_{m}}\right)^{\frac{3}{2}}\right) \int_{0}^{\frac{r}{r_{m}}} \frac{r_{m}^{2} \rho_{m} \ln \left(1+x^{\frac{3}{2}}\right)}{x^{\frac{7}{2}}\left(1+x^{\frac{3}{2}}\right)} d x
$$

\subsubsection{Halo pseudo-isotérmico}

O halo esférico pseudo-isotérmico difere do NFW por apresentar uma região central com densidade aproximadamente constante. Seu perfil é dado por:

$$
\rho(r)=\frac{\rho_{0}}{1+\left(\frac{r}{r_{c}}\right)^{2}},
$$


onde $\rho_{0}$ é a densidade central do halo e $r_{c}$ é o raio do "caroço" de densidade constante. Nesse caso, a velocidade circular de rotação é dada por:

$$
v_{c}(r)=\sqrt{4 \pi G \rho_{0} r^{2}\left[1-\left(\frac{r_{c}}{r}\right) \arctan \left(\frac{r}{r_{c}}\right)\right]} .
$$

Esse halo fica completamente caracterizado por 2 dentre os 3 parâmetros a seguir: $r_{c}, \rho_{0}$ e $v_{\infty}$, esse último uma velocidade assintótica, dada por (3.31) no limite em que $r \rightarrow \infty$ :

$$
v_{\infty}=\sqrt{4 \pi G \rho_{0} r^{2}}
$$

A massa contida dentro de um raio r é por sua vez:

$$
M(r)=4 \pi \int_{0}^{r} \frac{\rho_{0} r^{\prime 2}}{1+\left(\frac{r^{\prime}}{r_{c}}\right)^{2}} d r^{\prime}=4 \pi \rho_{0} r_{0}^{2}\left[r-r_{c} \arctan \left(\frac{r}{r_{c}}\right)\right] .
$$

A tabela (3.1) resume os resultados encontrados:

\begin{tabular}{|c|c|c|c|c|}
\hline & $\alpha$ & $\beta$ & $\gamma$ & $\mathrm{R}$ \\
\hline \hline NFW & 1.0 & 3.0 & 1.0 & $*$ \\
\hline Moore et al & 1.5 & 3.0 & 1.5 & $*$ \\
\hline Pseudo-Isotérmico & 2.0 & 2.0 & 0 & $*$ \\
\hline Isotérmico & - & 2.0 & 2.0 & $*$ \\
\hline
\end{tabular}

Tabela 3.1: Parâmetros (3.17) para alguns dos perfis mais utilizados para modelagem da densidade dos halos escuros em galáxias. Os valores de $\mathrm{R}\left(r_{m}, r_{s}\right.$ ou $\left.r_{c}\right)$ variam de galáxia para galáxia.

\subsubsection{Considerações sobre os perfis de densidade}

Os chamados raios característicos $r_{s}$ e $r_{m}$ dos perfis de NFW e Moore et al. podem ser relacionados se impusermos a condição de que eles tenham um pico para a velocidade circular $\left(v=v_{\max }\right)$ no mesmo lugar, digamos $r=r_{\max }$. Procurando pelos máximos de (3.24) e (3.28), encontramos respectivamente: $r_{\max } \simeq 2.16 r_{s}$ para o primeiro caso e $r_{\text {max }} \simeq 1.24 r_{m}$ no segundo, o que nos fornece $r_{m}(\widetilde{c})=$ $0.574 r_{s}(c)$.

Claramente, os perfis de NFW e Moore et al. diferem pouco quando lidamos com raios grandes (ambos apresentam comportamento $\rho \propto r^{-3}$ ), embora o mesmo não possa ser dito quando analisamos o comportamento assintótico $r \rightarrow 0$. Entretanto, como já foi apontado em várias referências, [?, ?, ?, ?], o mínimo raio para o qual uma distribuição de massa pode ser confiavelmente estimada em simulações numéricas deve ser tomado com extrema cautela. Um dos motivos principais 
é sem dúvida o fato de que as simulações trabalham com um número finito de partículas, o que acaba impondo limites severos para o poço de potencial capaz de ser gerado nesses casos. Dessa forma, para raios muito pequenos, os ajustes para a distribuição de massa acabam sendo fortemente dominados por efeitos numéricos e conclusões sobre as diferenças dos dois modelos não são exatamente triviais.

Já com respeito aos modelos que apresentam uma densidade central constante, os problemas repousam sempre na dúvida do porquê desse tipo de perfil, embora aparentemente mais consistente com dados de galáxias anãs e LSB, não ser o preferido pelas simulações numéricas. Tentativas recentes de conciliar os dois resultados têm sugerido aumentar a complexidade das interações nesses halos, por exemplo contabilizando fenômenos de reacreção de massa pelas galáxias [?], ou mesmo a suposição de uma matéria escura auto-interagente com seção de choque pequena mas não nula [?], mas nenhum resultado definitivo parece ter surgido até então.

\subsection{Crise no modelo CDM devido ao perfil de den- sidades de halos escuros}

A existência de um perfil do tipo "core" ou "cusp" no centro dos halos tem uma importância mais fundamental do que talvez se possa imaginar. Pistas sobre a natureza da matéria escura podem ser extraídas dos modelos que tratam disso e, na realidade, esse tipo de dúvida é em grande parte responsável pelo crescente número de modelos alternativos que tentam justificar os perfis dos halos. Questões como as levantadas pelo estudo das curvas de rotação, sugerem alternativas diversas, passando até mesmo por alterações da gravitação newtoniana para grandes escalas [?].

Em resumo, o que acontece é que o modelo $\Lambda C D M$ é de fato bastante bem sucedido quando procura explicações de fenômenos que ocorrem em largas escalas (da ordem de $10 \mathrm{Mpc}$ ou maiores). Quando o objetivo é fazer previsões em escalas menores (algo em torno de $1 \mathrm{Mpc}$ ou menos) a situação é um pouco diferente. A falta de vínculos experimentais sobre as possíveis partículas constituintes da matéria escura, sejam eles vindos de experimentos programados para uma deteç̧ão direta ou mesmo da observação de raios cósmicos, acaba por deixar uma porta aberta para especulações e descrições alternativas para uma parte da chamada "massa faltante" do universo.

$\mathrm{Na}$ realidade, a análise dos halos de matéria escura apresenta ainda mais alguns problemas [?]. No caso de galáxias em que a população bariônica não é desprezível, outra previsão que não se mostra condizente com dados experimentais é que a densidade de matéria escura, de acordo com as simulações, deveria ser muito maior nos centros dos halos galácticos em comparação com suas porções 
mais exteriores. Contudo, o que se observa é que muitas galáxias parecem ser dominadas nas regiões próximas de seus centros por bárions, sendo que uma parcela razoável do conteúdo de matéria escura se localiza nas porções mais externas. Existem algumas referências que abordam o problema sob essa ótica, como [?], fazendo simulações que incluem matéria bariônica e matéria escura. A princípio, poderia se imaginar que a inclusão dessas duas componentes poderia ser uma solução para o perfil de densidades suave encontrado nas galáxias, mas essa hipótese também parece já ter sido descartada por outros trabalhos [?]. Outro ponto freqüentemente atacado e ainda sem solução aparente é que o número de galáxias-satélite que deveria existir de acordo com as simulações, em torno de cada halo galáctico, é muito maior do que o que é visto, por exemplo, na Via-Láctea.

\subsection{Natureza da matéria escura}

Até aqui, discutimos os possíveis desdobramentos obtidos ao se supor uma matéria escura do tipo fria (CDM) e suas eventuais implicações para os perfis de densidade de galáxias e aglomerados. Entretanto, tendo em vista os problemas apresentados, é interessante saber se existem outros candidatos promissores e, se não, quais os motivos que os desqualificam.

Usualmente, a matéria escura, ao menos para efeitos didáticos, é dividida em duas porções, uma bariônica (BDM), outra não-bariônica (NBDM). Dentro da porção bariônica, os candidatos mais referendados, muitos deles também conhecidos pela alcunha genérica de MACHOs ("massive compact halo objects) são:

- Estrelas de baixa-luminosidade e remanescentes estelares;

- Pequenos corpos sólidos;

- Gás neutro ou ionizado;

- Buracos negros massivos.

Geralmente, uma forma de detectar a presença desses objetos consiste no estudo do efeito de microlentes gravitacionais, que já deram grandes contribuições nesse sentido [?, ?], detectando a presença de vários desses elementos. Entretanto, embora dados atuais (abundâncias de elementos leves observadas hoje) reiterem a existência de matéria escura nessa forma, os mesmos impõem vínculos severos quanto à quantidade de BDM que pode existir. Esse vínculo, advindo da nucleossíntese primordial, é bastante forte e, combinado com dados da RCF e de supernovas do tipo IA, contesta fortemente a possibilidade de a matéria escura bariônica sozinha ser capaz de explicar dados provenientes das observações atuais [?], como um universo com seção espacial plana. 
Já para o caso de matéria escura não-bariônica, existe uma primeira subdivisão entre matéria escura quente e fria, dependendo de essas partículas serem ou não relativísticas durante a época de desacoplamento.

Durante algum tempo, se especulou sobre a possibilidade de a matéria escura quente, por exemplo neutrinos relativísticos, ser a porção faltante da massa do universo mas, devido aos severos vínculos observacionais para a massa dos neutrinos e por inconsistências com cenários de formação de estruturas (torna-se difícil explicar a existência de certas galáxias e quasares com altos redshifts), isso vem sendo descartado [?].

Quanto ao cenário de matéria escura fria, durante algum tempo parece ter havido uma espécie de consenso global sobre a sua natureza. Ela deveria ser composta de partículas massivas fracamente interagentes (WIMPS), como por exemplo o neutralino, que aparece no contexto da chamado Modelo Padrão Mínimo Supersimétrico (MSSM) [?], tendo uma massa esperada da ordem de dezenas a centenas de $\mathrm{GeV}$. Entretanto, o fato de essa partícula ainda não ter sido detectada afasta aos poucos essa possibilidade. Um candidato também freqüentemente sugerido, o áxion, previsto no contexto da violação CP no modelo padrão de partículas desfruta hoje de igual condição, não tendo ainda sido detectado. Mais detalhes podem ser encontrados por exemplo na referência [?].

Essa coleção de resultados fornece portanto um sugestivo panorama para o começo de um estudo de possibilidades alternativas à matéria escura fria composta por partículas acolisionais. No capítulo posterior, será detalhado um "toymodel" sobre evolução de um colapso esférico dessas partículas, bastante representativo de alguns problemas apresentados pelo modelo CDM. Segue-se a isso a introdução de algumas idéias recentes de modelagem da matéria escura, notadamente as que usam campos escalares. 


\section{Capítulo 4}

\section{Modelagem dos halos}

Neste capítulo serão apresentados alguns modelos que sugerem propostas para o tratamento analítico ou semi-analítico da evolução dos halos de matéria escura. O primeiro deles, conhecido como modelo do colapso esférico não-linear, trabalha amplamente apoiado no paradigma CDM, com a hipótese de a matéria escura ser composta por partículas que interagem apenas gravitacionalmente. Não sendo essa a abordagem adotada por nós no trabalho, ele não será discutido em muitos detalhes, apenas servirá como uma boa fonte de noção intuitiva do problema. Versões mais incrementadas do modelo do colapso esférico existem em um número relativamente grande e podem por exemplo ser encontradas em [?]. Entretanto, devido à sua relativa simplicidade, ele se mostrará particularmente interessante por proporcionar uma clara visão esquemática da situação e sugerir um possível mecanismo de formação dos halos.

Posteriormente, apresentaremos dois modelos alternativos, que adotam a hipótese de a matéria escura ser composta não por partículas, mas por campos escalares (SFDM). A idéia agora é buscar responder a seguinte questão: se supuséssemos uma métrica estática e esfericamente simétrica, quais seriam as possíveis soluções para um campo com lagrangeana tipo canônica capazes de simular uma configuração para o campo que respondesse pelas propriedades observadas nas curvas de rotação?

Os modelos são estruturados de maneiras distintas, com detalhes e peculiaridades que, levando a conclusões diferentes, serão discutidos em detalhe ao longo do texto.

\subsection{Modelos com matéria escura fria}

\subsubsection{O modelo do colapso esférico}

Para o desenvolvimento desse modelo, tomaremos por base o tratamento sugerido na referência [?]. 
O pano de fundo para o problema estudado é o seguinte: consideremos um universo preenchido por uma densidade média, dita densidade de background $\rho_{b}$. Suponhamos agora a existência de um certo constraste de densidade $\delta\left(\vec{x}, t_{i}\right)$ para o universo em algum instante $t_{i}$, de tal forma que a densidade total em algum ponto do espaço seja dada por:

$$
\rho\left(r, t_{i}\right)=\rho_{b}\left(t_{i}\right)+\delta \rho\left(r, t_{i}\right)=\rho_{b}\left(t_{i}\right)\left[1+\delta_{i}(r)\right],
$$

onde foi implicitamente acrescida a hipótese dessa perturbação (contraste) de densidade ser esfericamente simétrica, justamente o que dá nome ao modelo. $\mathrm{Na}$ presença desse contraste de densidade, podemos considerar o universo como separado em duas regiões distintas, ditas sobredensas $(\delta>0)$ e subdensas $(\delta<0)$. Para o nosso estudo, nos concentraremos na primeira delas que, intuitivamente, deve se comportar da seguinte maneira: para regiões com um excesso de densidade considerável, ocorrerá um colapso que será responsável pela formação de objetos ligados gravitacionalmente. Por sua vez, a própria gravidade dessa concentração de massa irá realizar um trabalho contra a expansão do universo, fazendo com que essa porção não acompanhe o mesmo ritmo de expansão que toda a porção restante (que caminha com o fluxo de Hubble). O efeito resultante será uma desaceleração que aumentará ainda mais a diferença de densidade entre a região sobredensa e o background, fazendo com que o potencial gravitacional se torne cada vez mais dominante nessa região.

A forma exata como se desencadeará esse processo depende do perfil de densidades inicial suposto para a região do universo em estudo. Em nosso caso, em concordância com (4.1), utilizaremos um perfil com simetria esférica.

\subsubsection{Cálculos do modelo}

Supondo estarmos interessados apenas em perturbações com $\lambda \ll d_{H}$, o tamanho $\mathrm{R}$ da região sobredensa pode ser tomado como muito menor que o raio de Hubble $\left(d_{H}\right)$ [?]. Sendo assim, podemos utilizar a aproximação Newtoniana para calcular a evolução das perturbações. Nesse regime, o movimento de uma camada esférica de partículas localizada a uma distância $\mathrm{r}$ da origem é governado pela seguinte equação:

$$
\frac{d^{2} \vec{r}}{d t^{2}}=-\vec{\nabla} \Psi=-\frac{G M_{b}}{r^{3}} \vec{r}-\frac{G \delta M(r, t)}{r^{3}} \vec{r},
$$

onde $M_{b}$ é a massa contida em uma esfera de raio $\mathrm{r}$ devido à densidade do background e $\delta M(r, t)$ é a massa contida em uma esfera de raio $r(r=a(t)|\vec{x}|$, onde $\vec{x}$ é a coordenada comóvel) proveniente da perturbação de densidade do background. De uma maneira mais precisa, temos:

$$
M_{b}=\frac{4 \pi}{3} \rho_{b}(t) r^{3}=\frac{4 \pi}{3} \rho_{b}(t) a^{3}(t) x^{3},
$$




$$
\delta M(r, t)=4 \pi \int_{0}^{r} \delta \rho\left(r^{\prime}, t\right) r^{\prime 2} d r^{\prime}=4 \pi \rho_{b}(t) \int_{0}^{r} r^{\prime 2} \delta\left(r^{\prime}, t\right) d r^{\prime}
$$

Nesse ponto, chega-se um detalhe que merece especial atenção. Utiliza-se uma hipótese absolutamente crucial para o desenrolar do desenvolvimento do modelo e que simplifica sobremaneira os cálculos. A hipótese é a seguinte: suponhamos que as camadas esféricas estudadas não se cruzam durante a evolução do processo, ou seja, se colocássemos uma espécie de rótulo (aqui se entende $r$ como a etiqueta da casca $\delta M_{r, t_{i}}$ ) em cada uma das camadas, de tal forma que elas fossem por exemplo agrupadas em ordem crescente de raio, essa ordenação inicial não se alteraria no decorrer do tempo. Como conseqüência, a massa contida em uma camada de raio $R$ é sempre constante, o que é fácil de ser compreendido, já que nesse caso as partículas inicialmente em uma determinada camada não passam para outras. Essa condição pode ser expressa na forma:

$$
\delta M(r, t)=\delta M\left(r, t_{i}\right) .
$$

Podemos então utilizar (4.4) e reescrever a equação (4.2) :

$$
\frac{d^{2} \vec{r}}{d t^{2}}=-\frac{G M}{r^{3}} \vec{r}
$$

sendo que agora, se supusermos $r_{i}$ como o raio inicial da camada com raio $\mathrm{M}$ teremos:

$$
\begin{aligned}
& M=\rho_{b}\left(\frac{4 \pi}{3} r_{i}^{3}\right)\left(1+\bar{\delta}_{i}\right), \\
& \bar{\delta}_{i}=\frac{3}{r_{i}^{3}} \int_{0}^{r_{i}} \delta_{i}(r) r^{2} d r .
\end{aligned}
$$

a última igualdade indicado a média de $\delta_{i}$ no volume.

A equação (4.5) pode ser integrada, fornecendo uma integral do movimento que chamaremos energia $(\mathrm{E})$ :

$$
\frac{1}{2}\left(\frac{d r}{d t}\right)^{2}-\frac{G M}{r}=E .
$$

De $(4.7)$ é fácil ver que $\left(\frac{d r}{d t}\right)$ pode mudar de sinal para $E<0$, ou seja, uma perturbação que inicialmente se expande pode eventualmente se contrair.

Olhemos agora para a solução da equação (4.7) e vejamos quais conclusões podem ser tiradas. $\mathrm{Na}$ análise que vem a seguir, suporemos que as partículas das camadas esféricas, além de serem afetadas pelo fluxo de Hubble, também têm uma velocidade peculiar inicial diferente de zero, ou seja, para $t=t_{i}$ temos:

$$
\dot{r}_{i}=H_{i} r_{i}+v_{i}\left(r_{i}\right) .
$$

O passo seguinte consiste em encontrar o valor da "energia" (E), que aparece em (4.7) a partir das condições iniciais. Ficamos com: 


$$
\begin{gathered}
K_{i}=\left.\frac{\dot{r}^{2}}{2}\right|_{t=t_{i}}=\frac{H_{i} r_{i}^{2}}{2}+H_{i} r_{i} v_{i}+\frac{v_{i}^{2}}{2} \\
\left|U_{i}\right|=\left.\frac{G M}{r}\right|_{t=t_{i}}=\frac{4 \pi}{3}\left(1+\delta_{i}\right) G \rho_{b}\left(t_{i}\right)=\frac{1}{2} H_{i}^{2} r_{i}^{2}\left(1+\bar{\delta}_{i}\right) \frac{\rho_{b}\left(t_{i}\right)}{\rho_{c}}=\frac{H_{i} r_{i}^{2}}{2} \Omega_{i}\left(1+\bar{\delta}_{i}\right),
\end{gathered}
$$

onde $\Omega_{i}$ é a razão entre a densidade (inicial) do background e a densidade crítica. Agrupando os dois termos em (4.8), (4.9) e lembrando que a energia (E) se conserva, podemos finalmente escrever:

$$
E=E_{i}=K_{i}-\left|U_{i}\right|=-\frac{H_{i} r_{i}^{2}}{2} \Omega_{i}\left[\left(1+\bar{\delta}_{i}\right)-\frac{1}{\Omega_{i}}\left(1+\frac{v_{i}}{H_{i} r_{i}}\right)^{2}\right]=-\frac{H_{i} r_{i}^{2}}{2} \Omega_{i} D
$$

sendo D obviamente igual a :

$$
D=\left(1+\bar{\delta}_{i}\right)-\frac{1}{\Omega_{i}}\left(1+\frac{v_{i}}{H_{i} r_{i}}\right)^{2}
$$

Novamente da equação (4.7), podemos ver que condições devemos ter para que ocorra $E<0$, ou seja, buscamos uma condição que nos permita a ocorrência de um colapso das camadas. De (4.10) é imediato ver que isto ocorre no caso em que $D>0$, ou seja:

$$
\bar{\delta}_{i}>\frac{1}{\Omega_{i}}\left(1+\frac{v_{i}}{H_{i} r_{i}}\right)^{2}-1 .
$$

Olhemos agora para o raio máximo $\left(r_{\max }\right)$ que uma camada esférica evoluindo de acordo com (4.7) pode atingir. Ora, isso tão-somente equivale a olharmos para (4.7) no momento em que $\dot{r}=0$, ou seja, quando a energia cinética da camada de raio $r=r_{\max }$ é nula. Ficamos com:

$$
E=-\frac{G M}{r_{\max }}=-\frac{H_{i}^{2} r_{i}^{2} \Omega_{i}}{2} D
$$

Ou seja :

$$
r_{\max }=\frac{2 G M}{H_{i}^{2} r_{i}^{2} \Omega_{i} D}=\frac{1+\bar{\delta}_{i}}{D} r_{i} .
$$

Esse momento, em que uma dada camada atinge seu tamanho máximo, é conhecido por um nome especial: turn-around. 


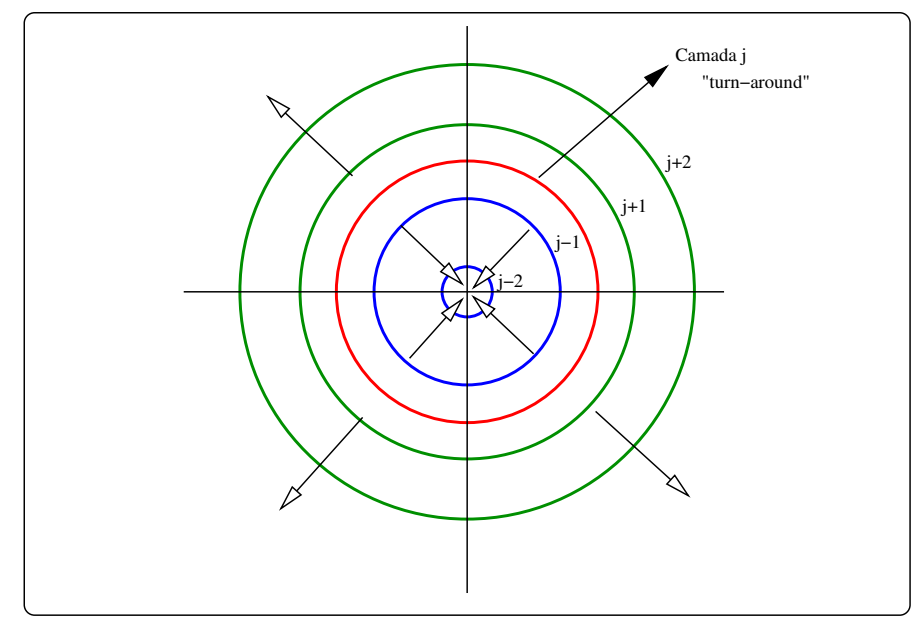

Figura 4.1: Representação esquemática (bidimensional) da evolução das camadas. As camadas azuis já passaram pelo turn-around e agora apenas diminuem seu volume, o contrário valendo para as verdes. A camada vermelha, indicada por $\mathrm{j}$ no desenho, aparece no exato momento em que atinge $r_{\max }$.

Considerações iniciais feitas, podemos partir para a solução da equação (4.7) propriamente dita. Em uma forma paramétrica, podemos escrevê-la na forma:

$$
r=A(1-\cos \theta), t-T=B(\theta-\sin \theta), A^{3}=G M B^{2} .
$$

Por sua vez, as constantes em (4.13) podem ser encontradas em função das condições iniciais. Da equação para $r$, vemos que o raio máximo ocorre para $\theta=\pi$. Nesse caso temos:

$$
r_{m}=2 A==>A=\frac{r_{m}}{2}=\frac{r_{i}}{2 D}\left(1+\bar{\delta}_{i}\right) .
$$

Como $A^{3}=G M B^{2}$, temos para B:

$$
B=\frac{A^{\frac{3}{2}}}{\sqrt{G M}}=\left(\frac{r_{i}}{2 D}\right)^{\frac{3}{2}} \frac{1}{\sqrt{G M}}\left(1+\bar{\delta}_{i}\right)^{\frac{3}{2}} .
$$

Olhando para (4.12) podemos simplificar ainda mais essa expressão obtendo:

$$
B=\left(\frac{r_{i}}{2 D}\right)^{\frac{3}{2}}\left(1+\bar{\delta}_{i}\right)^{\frac{3}{2}} \frac{\sqrt{2}}{H_{i} r_{i}\left(\Omega_{i} D r_{m}\right)^{\frac{1}{2}}} .
$$

Utilizando agora a expressão obtida para o raio máximo da camada esférica, (4.14), ficamos com:

$$
B=\frac{1+\bar{\delta}_{i}}{2 H_{i} \Omega_{i}^{\frac{1}{2}} D^{\frac{3}{2}}}
$$


Além dessas condições, também temos um vínculo para $T$, que nos permite fixar a condição de que, para $t=t_{i}, r=r_{i}$. Temos então:

$$
T=t_{i}-B\left(\theta_{i}-\sin \theta_{i}\right) ; r_{i}=A\left(1-\cos \theta_{i}\right) .
$$

A fim de prosseguirmos na análise, restrinjamos um pouco mais nosso modelo, olhemos para o que acontece para o contraste de densidade em um universo de Einstein-De Sitter, portanto com $\mathrm{k}=0$, fator de escala $a \propto t^{\frac{2}{3}}$ e com $\rho_{b}=\frac{1}{6 \pi G t^{2}}$ - Se olharmos agora para a razão entre a densidade média $(\bar{\rho})$ e a densidade do background $\left(\rho_{b}\right)$, encontramos a seguinte relação:

$$
\frac{\bar{\rho}}{\rho_{b}}=1+\bar{\delta}(r, t)=\frac{3 M}{4 \pi r^{3}} 6 \pi G t^{2}=\frac{3 M}{4 \pi A^{3}(1-\cos \theta)^{3}} 6 \pi G B^{2}\left(\theta-\sin \theta+\frac{T}{B}\right)^{2} .
$$

Como $A^{3}=G M B^{2}$, simplificando, ficamos com:

$$
\bar{\delta}=\frac{9}{2} \frac{\left(\theta-\sin \theta+\frac{T}{B}\right)^{2}}{(1-\cos \theta)^{3}}-1 .
$$

Podemos agora extrair alguns resultados interessantes se nos preocuparmos com o comportamento de $\bar{\delta}$ no limite em que $(t-T)$ é pequeno.

Uma expansão da equação (4.19) para $\theta$ pequeno nos fornece, com a ajuda de (4.13):

$$
\bar{\delta} \approx \frac{9}{2\left(\frac{\theta^{2}}{2 !}-\frac{\theta^{4}}{4 !}\right)^{3}}\left[\left(\frac{\theta^{3}}{3 !}-\frac{\theta^{5}}{5 !}\right)^{2}+\frac{2 T}{B}\left(\frac{\theta^{3}}{3 !}-\frac{\theta^{5}}{5 !}\right)+\frac{T^{2}}{B^{2}}\right]-1
$$

Desprezando termos de ordem superior:

$$
\bar{\delta}=\frac{3}{20}\left[\frac{6(t-T)}{B}\right]^{\frac{2}{3}}+\frac{2 T}{(t-T)} .
$$

Por outro lado, no caso em que estamos analisando também temos $\Omega_{i}=1$, logo $H_{i}=\frac{2}{3 t_{i}}$, de modo que podemos escrever, em ordem dominante, utilizando (4.17):

$$
B=\frac{3}{4} \frac{t_{i}}{\left(\bar{\delta}_{i}-3 \frac{t_{i} v_{i}}{r_{i}}\right)^{\frac{3}{2}}} .
$$

Podemos então injetar o valor de B de (4.21) na expressão (4.19), obtendo finalmente:

$$
\bar{\delta}=\frac{3}{5}\left(\frac{t}{t_{i}}\right)^{\frac{2}{3}}\left[\bar{\delta}_{i}-\frac{3 t_{i} v_{i}}{r_{i}}\right]+\frac{2}{5}\left(\frac{t_{i}}{t}\right)\left[\bar{\delta}_{i}+\frac{9 t_{i} v_{i}}{2 r_{i}}\right]
$$


É bastante interessante agora olharmos para alguns limites dessa solução encontrada e ver quais conclusões podem ser tiradas a respeito dela. Um caso particularmente interessante e analiticamente solúvel aparece no limite em que as velocidades peculiares iniciais são zero. Nesse caso, as equações se simplificam bastante, já que podemos provar que $T$ pode ser desprezado nesta situação. A condição imposta por (4.18) assume assim a forma:

$$
\begin{gathered}
r_{i}=\frac{r_{i}}{2}\left(\frac{1+\bar{\delta}_{i}}{\bar{\delta}_{i}}\right)\left(1+\cos \theta_{i}\right), \\
t_{i}+T=\frac{1}{2 H_{i}}\left(\frac{1+\bar{\delta}_{i}}{\bar{\delta}_{i}^{\frac{3}{2}}}\right)\left(\theta_{i}-\sin \theta_{i}\right) .
\end{gathered}
$$

De (4.23), fazendo uma aproximação baseada na hipótese que $\delta_{i}$ é pequeno, encontramos $\theta_{i}^{2} \approx 4 \delta_{i}$. Substituindo em (4.24):

$$
H_{i}\left(t_{i}+T\right)=\frac{2}{3}\left(1+\delta_{i}\right) .
$$

Para o universo de De Sitter (Apêndice B), $H_{i} t_{i}=\frac{2}{3}$. Isso permite escrever $H_{i} T=\frac{2}{3} \bar{\delta}_{i}$, ou seja:

$$
\frac{T}{t_{i}}=\bar{\delta}_{i} \ll 1,
$$

permitindo assim ignorar $\mathrm{T}$ no que segue.

Se, além disso, supusermos que $\bar{\delta}_{i}$ é pequeno o bastante em comparação com a unidade, obtemos:

$r=\frac{r_{i}}{2 \bar{\delta}_{i}}(1-\cos \theta)=\frac{3 X}{10 \bar{\delta}_{0}}(1-\cos \theta) ; t=\frac{3 t_{i}}{4 \bar{\delta}_{i}^{\frac{3}{2}}}(\theta-\sin \theta)=\left(\frac{3}{5}\right)^{\frac{3}{2}} \frac{3 t_{0}}{4 \bar{\delta}_{0}^{\frac{3}{2}}}(\theta-\sin \theta)$,

onde se escreveram as quantidades em termos do raio comóvel $X=r_{i} \frac{a\left(t_{0}\right)}{a\left(t_{i}\right)}$ correspondente a $r_{i}$ e de $\bar{\delta}_{0}=\frac{a\left(t_{0}\right)}{a\left(t_{i}\right)}\left(\frac{3}{5}\right) \bar{\delta}_{i}$, que nada mais é que o valor atual do contraste de densidade que seria previsto pela teoria linear ${ }^{1}$ se o contraste de densidade inicial fosse $\bar{\delta}_{i}$ no redshift $z_{i}$.

Lembrando ainda que, nesse universo, $\left(\frac{t}{t_{i}}\right)^{\frac{2}{3}}=\left(1+z_{i}\right)(1+z)^{-1}$, podemos estabelecer uma relação entre $\theta$ e o redshift $z$, utilizando (4.25):

$$
(1+z)=\left(\frac{4}{3}\right)^{\frac{2}{3}} \frac{\bar{\delta}_{i}\left(1+z_{i}\right)}{(\theta-\sin \theta)^{\frac{2}{3}}}=\left(\frac{5}{3}\right)\left(\frac{4}{3}\right)^{\frac{2}{3}} \frac{\overline{\delta_{0}}}{(\theta-\sin \theta)^{\frac{2}{3}}}
$$

\footnotetext{
${ }^{1}$ Vide Apêndice A.
} 
Se olharmos novamente para (4.22), agora com $T$ e $v$ supostos desprezíveis, podemos escrever:

$$
\bar{\delta}=\frac{3}{5}\left(\frac{t}{t_{i}}\right)^{\frac{2}{3}} \bar{\delta}_{i} .
$$

Ou seja, o resultado que é obtido via teoria linear de perturbações para um modo puramente crescente, $\delta_{L}$, mostrando que a abordagem é consistente, redescobrimos o $\delta$ correto quando o intervalo de tempo é pequeno. Utilizando (4.26):

$$
\delta_{L} \equiv \frac{\bar{\rho}_{L}}{\rho_{b}}-1=\frac{3}{5} \frac{\bar{\delta}_{i}\left(1+z_{i}\right)}{1+z}=\frac{3}{5}\left(\frac{3}{4}\right)^{\frac{2}{3}}(\theta-\sin \theta)^{\frac{2}{3}} .
$$

Tal resultado rapidamente nos convida a uma comparação entre as duas abordagens, o que pode ser obtido se olharmos para os valores de $\delta_{L}$ e $\delta$ em algumas situações interessantes. Por exemplo, no limite em que $z \ll 1$, teremos $\theta \ll 1 \mathrm{e}$ um resultado concordante para os dois modelos $\bar{\delta} \approx \delta_{L}$. Por outro lado, quando $\theta=\frac{\pi}{2}, \delta_{L}=0.341$ e $\bar{\delta}=0.466$, ou seja, uma diferença de cerca de $40 \%$. Para $\theta=\frac{2 \pi}{3}, \delta_{L}=0.568$ e $\bar{\delta}=1.01 \approx 1$. Interpretando esse resultado $(\bar{\delta} \approx 1)$ como a transição para a não-linearidade, podemos ver de (4.19) que isso acontece para um redshift de: $\left(1+z_{n l}\right)=1.06 \bar{\delta}_{i}\left(1+z_{i}\right)=\frac{\bar{\delta}_{0}}{0.57}$. Por fim, a região esférica atingirá seu tamanho máximo quando tivermos atingido a situação $\theta=\pi$. Neste caso, podemos inferir:

$$
\begin{aligned}
\left(1+z_{m}\right) & =\left(\frac{4}{3}\right)^{\frac{2}{3}} \frac{\bar{\delta}_{i}\left(1+z_{i}\right)}{\pi^{\frac{2}{3}}}=0.57\left(1+z_{i}\right) \bar{\delta}_{i} \\
& =\frac{5}{3}\left(\frac{4}{3 \pi}\right)^{\frac{2}{3}} \bar{\delta}_{0} \approx \frac{\bar{\delta}_{0}}{1.062} \\
r_{m} & =\frac{3 X}{5 \bar{\delta}_{0}} \\
1+\bar{\delta}_{m} & =\frac{9 \pi^{2}}{16} \approx 5.6
\end{aligned}
$$

onde o subscrito $m$ corresponde aos valores dessas quantidades quando a região esférica atinge seu tamanho máximo.

A interpretação física dessas soluções é relativamente simples. A primeira delas, (4.29), mostra quando uma camada esférica inicialmente situada no redshift $z_{i}$ (onde existe um contraste inicial de densidade $\delta_{i}$ ), irá cessar sua expansão ("turn around"). Já a segunda, (4.30), fornece o raio máximo atingido pela camada esférica. A última delas, (4.31), responde quantas vezes a região será mais densa que o background (novamente no "turn-around"). Logo após atingir seu raio máximo, a camada esférica continuará seu processo de contração e, da equação 
(4.25), podemos ver que isso continuaria ocorrendo até atingirmos uma situação em que $\theta=2 \pi$, ou seja, teríamos toda a matéria colapsada em um único ponto. Só que é exatamente nesse período que aparece uma das grandes complicações desse modelo. Essa contração precisa cessar de alguma forma, até porque sabemos que a aproximação utilizada, de que a matéria se organizará em camadas esféricas que não se cruzam, não necessariamente continuará sendo válida. A teoria de sistemas acolisionais, como é o caso da matéria escura nesse modelo, nos diz que, antes do colapso puntual acontecer, haverá um processo de virialização [?], com a região esférica atingindo uma configuração com raio $r_{v i r}$, dispersão de velocidades $v$ e densidade $\rho_{c o l}$. Baseados nisso, se utilizarmos posteriormente o teorema de virial (4.32), podemos extrair uma expressão para a energia que o sistema terá depois de atingir a estabilidade. A energia total será: $E=U+K=-K$. Para $t=t_{m}$, toda a energia estará na forma de energia potencial e, como estamos lidando com um sistema esfericamente simétrico, é simples calcular a energia armazenada nessa configuração $\left(E \approx \frac{-3 G M^{2}}{5 r}\right)$. Pode-se estimar os valores para $v$ e $r_{v i r}$ nestas condições:

$$
\begin{aligned}
K & =\frac{M v^{2}}{2}=-E=\frac{3 G M^{2}}{5 r_{m}} \\
V & =\frac{3 G M^{2}}{5 r_{v i r}}=2 K=M v^{2} .
\end{aligned}
$$

A equação (4.32) nos permite obter:

$$
v=\left(\frac{6 G M}{5 r_{m}}\right)^{\frac{1}{2}}, r_{v i r}=\frac{r_{m}}{2}
$$

O tempo estimado para o sistema atingir o equilíbrio de virial é essencialmente $t_{c o l}$, ou seja, o tempo quando $\theta=2 \pi$. Da equação (4.26) notamos que isso acontece para:

$$
\left(1+z_{\mathrm{col}}\right)=\left(\frac{4}{3}\right)^{\frac{2}{3}} \frac{\bar{\delta}_{i}\left(1+z_{i}\right)}{(2 \pi)^{\frac{2}{3}}}=0.36 \bar{\delta}_{i}\left(1+z_{i}\right)=0.63\left(1+z_{m}\right)=\frac{\bar{\delta}_{0}}{1.686}
$$

Além desse resultado, a densidade do objeto colapsado também pode ser obtida. Basta lembrar que $r_{v i r}=\frac{r_{m}}{2}$. Logo, $\rho_{c o l}=8 \rho_{m}$. Por outro lado, já mostramos que $\rho_{m}$ é da ordem de 6 vezes a densidade do background $\left(\rho_{m} \approx\right.$ $\left.5.6 \rho_{b}\left(t_{m}\right)\right)$. Por sua vez, $\rho_{b}\left(t_{m}\right)=\frac{\left(1+z_{m}\right)^{3}}{\left(1+z_{c o l}\right)^{3}} \rho_{b}\left(t_{c o l}\right)$. Resumindo, chegamos ao seguinte resultado:

$$
\rho_{c o l}=8 \rho_{m}=(5.6)(8) \rho_{b}\left(t_{m}\right) \approx 170 \rho_{b}\left(t_{c o l}\right) \approx 170 \rho_{0}\left(1+z_{c o l}\right)^{3} .
$$


Esse resultado é importante porque permite determinar $\rho_{c o l}$ como função do redshift de formação do objeto ligado. Uma vez que o sistema está virializado, sua densidade e seu tamanho não mudam mais. Dessa maneira, como $\rho_{b} \propto a^{-3}$, o contraste de densidade $\bar{\delta}$ crescerá com $a^{3}$ para $t>t_{\text {col }}$.

A presença do fator 170 em (4.36) esclarece o fato de se trabalhar tanto nos modelos numéricos com as quantidades escritas em função de $r_{200}$, como foi visto no capítulo 3.

\subsection{Modelos com campos escalares}

\subsubsection{Halo escalar esférico em galáxias}

Não são poucos os modelos utilizados para tentar solucionar a questão dos halos de matéria escura. Dentre esses, alguns que aparecem atualmente de forma relativamente promissora são aqueles que tentam modelar a matéria escura por intermédio de campos escalares. Em modelos dessa natureza, explora-se a possibilidade desse campo sofrer variações, flutuar ao longo da história do universo de modo a justificar os dados observacionais já conhecidos. Alguns resultados interessantes nesta área já começam a aparecer, como pode ser visto por exemplo nas referências [?, ?, ?, ?, ?, ?].

A idéia básica nesse tipo de abordagem consiste na exploração da possibilidade de que campos escalares possam fazer o papel de matéria escura no universo, assumindo para isso a hipótese de que a matéria escura escalar se distribui como um halo axialmente simétrico. Em alguns trabalhos já conhecidos na literatura [?], já foi, por exemplo, mostrado possível o campo escalar evoluir como matéria escura fria (CDM). Nesse último, prevê-se um perfil final de densidades do tipo NFW, do mesmo tipo mostrado em (3.18).

Vejamos agora como as duas abordagens se contrapõem e ressaltemos possíveis características interessantes dos modelos com campos escalares.

\subsubsection{Modelo CDM X Modelo SFDM}

Podemos primeiramente começar imaginando uma situação análoga à apresentada na seção anterior, com a diferença de que temos agora um campo escalar preenchendo o universo, com o mesmo apresentando algumas pequenas variações de um ponto para outro. Entretanto, o que acontece agora é que, quando as flutuações do campo escalar atingem o regime não linear, o campo escalar colapsa de uma maneira diferente daquela apresentada no modelo CDM padrão. Em um colapso normal ${ }^{2}$, não existe em princípio nada que possa impedir que a matéria colapse todo o tempo. Existe apenas uma força gravitacional radial que provoca o colapso e, para pará-lo, existe a necessidade de se invocar a presença de algum

\footnotetext{
${ }^{2}$ Equivalente a utilizar um tensor de energia-momento para partículas tipo poeira (dust)
} 
mecanismo de virialização, conforme apresentado na seção anterior. Vejamos o que acontece no caso do campo escalar, sabendo que em todo o procedimento descrito a seguir, suporemos um campo escalar acoplado à gravidade com ação na forma:

$$
S=\int d^{4} x \sqrt{-g}\left[-\frac{R}{16 \pi G}+\mathcal{L}(\Phi, \nabla \Phi)\right] .
$$

As definições obedecem a forma usual, $\mathrm{R}$ sendo o escalar de Ricci, g o determinante da métrica e $\mathcal{L}$ a densidade de lagrangeana.

O campo escalar canônico possui uma Lagrangeana dada por:

$$
L=\frac{1}{2} g^{\mu \nu} \partial_{\mu} \Phi \partial_{\nu} \Phi-V(\Phi)
$$

onde $V(\Phi)$ é o potencial para o campo.

Da teoria clássica de campos, sabemos que, dada uma densidade de lagrangeana $\mathcal{L}$, podemos extrair seu tensor de momento e energia a partir de [?]:

$$
T_{\mu \nu}=\frac{2}{\sqrt{-g}}\left[\frac{\partial(\sqrt{-g} \mathcal{L})}{\partial g^{\mu \nu}}-\frac{\partial}{\partial x^{\alpha}} \frac{\partial(\sqrt{-g} \mathcal{L})}{\partial g^{\mu \nu}{ }_{, \alpha}}\right] .
$$

Injetando (4.38) em (4.39), podemos calcular o tensor de momento e energia $T_{\mu \nu}$ :

$$
T_{\mu \nu}=\Phi_{, \mu} \Phi_{, \nu}-\frac{1}{2} g_{\mu \nu} \Phi^{, \sigma} \Phi_{, \sigma}-g_{\mu \nu} V(\Phi) .
$$

Da suposição de uma simetria esférica, o elemento de linha $\left(d s^{2}\right)$ tem forma (Apêndice A):

$$
d s^{2}=e^{2 \nu(r, t)} d t^{2}-e^{2 \mu(r, t)} d r^{2}-r^{2} d \Omega^{2} .
$$

O tensor de momento-energia do campo escalar canônico terá as seguintes componentes:

$$
\begin{gathered}
T_{0}^{0}=\rho_{\Phi}=\frac{1}{2}\left[e^{-2 \nu} \dot{\phi}^{2}+e^{-2 \mu} \Phi^{\prime 2}+2 V(\Phi)\right], \\
T^{0}{ }_{1}=\dot{\Phi} \Phi^{\prime}, \\
T_{1}^{1}=-p_{r}=\frac{1}{2}\left[-e^{-2 \nu} \dot{\Phi}^{2}-e^{-2 \mu} \Phi^{\prime 2}+2 V(\Phi)\right], \\
T_{2}^{2}=-p_{\theta}=\frac{1}{2}\left[-e^{-2 \nu} \dot{\Phi^{2}}+e^{-2 \mu} \Phi^{\prime 2}+2 V(\Phi)\right], \\
T^{3}{ }_{3}=T_{2}^{2} .
\end{gathered}
$$


As pressões radial e angular aparecem assim como duas componentes naturais do campo escalar capazes de parar o colapso, característica que pode evitar os perfis de densidade acentuados no centro de objetos colapsados, o que acaba aparecendo como uma grande diferença entre o colapso CDM normal (dust) e o SFDM.

\subsubsection{Modelos Mattos et al [?], [?]}

Quando o assunto é uma modelagem fenomenológica, a primeira abordagem que vem à mente é geralmente a mais simples. No caso dos campos escalares, um dos modelos mais estudados (excluído o caso de um potencial constante) consiste em um campo com um potencial do tipo $V(\Phi)=\frac{1}{2} m_{\Phi}^{2} \Phi^{2}$, em um background de Minkowski $(\mu \approx \nu \approx 0)$. É claro que essa ainda não é uma solução para as equações de Einstein, já que se trata de uma simplificação grosseira que despreza a força gravitacional provocada pelo campo mas, novamente por se tratar de uma solução analítica, é um bom ponto de partida para a compreensão de algumas características que aparecem em oscilações não-lineares.

Utilizando as equações de Euler-Lagrange:

$$
\frac{\partial \mathcal{L}}{\partial \Phi}-\frac{d}{d x^{\mu}}\left(\frac{\partial \mathcal{L}}{\partial\left(\partial_{\mu} \Phi\right)}\right)=0
$$

podemos obter a chamada equação de Klein-Gordon que, em um espaço esfericamente simétrico, fornece:

$$
\begin{gathered}
\square^{2}+\frac{d V(\Phi)}{d \Phi}=0, \\
\Phi^{\prime \prime}+\frac{2}{r} \Phi^{\prime}-m_{\Phi}^{2} \Phi=\ddot{\Phi},
\end{gathered}
$$

onde $\square=g^{\mu \nu} \nabla_{\mu} \nabla_{\nu}$. A solução exata para (4.47) é:

$$
\Phi(r, t)=\frac{e^{ \pm i k r}}{r} e^{ \pm i \omega t}
$$

com a relação de dispersão: $k^{2}=\omega^{2}-m_{\Phi}^{2}$. No caso em que $\omega>m_{\Phi}$ a solução é não-singular e tende a zero no infinito. Nesse caso, utilizando funções trigonométricas, podemos reescrever (4.48) na forma:

$$
\Phi(t, x)=\Phi_{0} \frac{\sin (x)}{x} \cos (\omega t),
$$

com $\Phi_{0}$ uma constante e $x=k r$.

A solução demonstra claramente que o campo oscila em todo o espaço, ou seja, não está confinado a uma região finita. Embora não seja uma solução 
física satisfatória, isso é razoável, se lembrarmos que a interação gravitacional foi desprezada até aqui.

Já a expressão para a densidade de energia do campo escalar é (4.42):

$$
\rho_{\Phi}=\frac{1}{2} \Phi_{0}^{2}\left[\left(\frac{\sin (x)-x \cos (x)}{x^{2}}\right)^{2}\right] k^{4} \cos ^{2} \omega t+\frac{1}{2} \Phi_{0}^{2} \frac{\omega^{2} k^{2} \sin ^{2}(x)}{x^{2}}
$$

que apresenta um comportamento para a região central do tipo:

$$
\rho_{\Phi} \approx \frac{1}{2} \Phi_{0}^{2} k^{2}\left[\omega^{2}-k^{2} \cos ^{2}(\omega t)\right]+O\left(x^{2}\right),
$$

ou seja, encontramos uma densidade quase constante para a região central (quando $x \rightarrow 0$, a densidade central oscila em torno de um valor fixo).

Já quando $x \rightarrow \infty$, o comportamento assintótico aparece na forma: $\rho_{\Phi} \approx \frac{1}{x^{2}}$, ou seja, algo do tipo esfera isotérmica (na realidade, a solução apresenta uma leve oscilação em torno de zero).

Prosseguindo na apresentação dos modelos, um passo um pouco adiante consiste no modelo apresentado em [?]. A preocupação agora é com a região mais externa do halo onde, como visto no capítulo anterior, as evidências para um perfil plano para as curvas de rotação de galáxias são mais claras e não há tantas controvérsias como no caso da região central. Nesse caso específico, a conexão do modelo com o fenômeno físico é realizada por intermédio de restrições feitas aos coeficientes da métrica do espaço-tempo na região das galáxias onde se observa um comportamento plano das curvas de rotação.

O procedimento é relativamente simples: primeiramente, determina-se a expressão para a velocidade tangencial de partículas-teste nesse espaço-tempo seguindo uma geodésica num movimento circular e estável (como uma função dos coeficientes da métrica). Posteriormente, impõe-se a parte fenomenológica, de que essa velocidade tangencial deve se mostrar independente do raio da trajetória das partículas, de modo a justificar o perfil plano desta região (mais "externa") das curvas de rotação. Esse último resultado permite a obtenção de uma equação de vínculo para os coeficientes da métrica, resultado que, conforme mostrado em [?], parece poder ser deduzido independentemente do tipo de matéria que gera a geometria do espaço-tempo em questão (ou seja, pode-se trabalhar com essa hipótese para fluidos perfeitos, campos escalares, constante cosmológica, etc).

Para esse caso, assumiremos, além de uma distribuição de massa esfericamente simétrica para o halo, um espaço-tempo estático, o que permite escrever um elemento de linha $d s^{2}$ da seguinte forma:

$$
d s^{2}=B(r) d t^{2}-A(r) d r^{2}-r^{2} d \theta^{2}-r^{2} \sin ^{2} \theta d \phi^{2} .
$$

Olhando agora para um movimento circular e estável de partículas-teste nesse espaço-tempo, seguindo o tratamento sugerido em [?] e aliando-se a isso a condição 
de um perfil plano para as curvas de rotação, restringimos ainda mais a métrica em (4.50), obtendo:

$$
\begin{gathered}
B(r)=B_{0} r^{l}, \\
l=2 v_{\phi}^{2},
\end{gathered}
$$

onde $B_{0}$ é uma constante e $v_{\phi}$ é a velocidade tangencial das partículas-teste.

Prosseguindo, podemos escrever as equações de Einstein e de Klein-Gordon para o campo, o que permite obter restrições também acerca do coeficiente A(r) da métrica (4.50). Utilizando (4.50), a equação de Klein-Gordon para o campo, suposto com um tensor de momento e energia como em (4.40), fornece:

$$
\frac{d^{2} \Phi}{d r^{2}}+\frac{1}{2 r}\left[l+4-\frac{1}{A} \frac{d A}{d r}\right] \frac{d \Phi}{d r}-\frac{1}{4} A \frac{d V(\Phi)}{d \Phi}=0 .
$$

Por outro lado, das equações de Einstein, obtemos:

$$
\begin{gathered}
\frac{A-(l+1)}{r^{2}}=-8 \pi G\left[\frac{1}{2}\left(\frac{d \Phi}{d r}\right)^{2}-A V(\Phi)\right], \\
\frac{1}{4 r^{2}}\left[l^{2}-\frac{1}{A} \frac{d A}{d r} r(l+2)\right]=-8 \pi G\left[\frac{1}{2}\left(\frac{d \Phi}{d r}\right)^{2}+A V(\Phi)\right], \\
\frac{1}{r^{2}}\left[1-A-\frac{1}{A} \frac{d A}{d r} r\right]=-8 \pi G\left[\frac{1}{2}\left(\frac{d \Phi}{d r}\right)^{2}+A V(\Phi)\right] .
\end{gathered}
$$

Fazendo uma manipulação algébrica dessas expressões: $(2-l) *(4.52)-4 *(4.53)$ $+(2+l) *(4.54)$, pode-se obter uma informação acerca do potencial escalar para uma métrica esfericamente simétrica aliada à condição de uma curva de rotação plana:

$$
V(r)=-\frac{l}{8 G(2-l)} \frac{1}{r^{2}}
$$

Ou seja, dessas equações, pode-se, inferir um potencial escalar que decai com o quadrado da distância.

Prosseguindo, a resolução das equações de campo fornece:

$$
A(r)=\frac{4-l^{2}}{4+C\left(4-l^{2}\right) r^{-(l+2)}},
$$

onde $\mathrm{C}$ aparece como uma constante de integração. Essa solução permite uma certa liberdade para o coeficiente $\mathrm{A}(\mathrm{r})$ da métrica. Uma possível solução pode ser por exemplo encontrada no caso simples em que $C=0$ :

$$
A=\frac{4-l^{2}}{4}
$$




$$
\begin{gathered}
\Phi=\sqrt{\frac{l}{8 \pi G}} \ln (r)+\Phi_{0}, \\
V(\Phi)=-\frac{l}{2-l} \exp \left[-2 \sqrt{\frac{8 \pi G}{l}}\left(\Phi-\Phi_{0}\right)\right] .
\end{gathered}
$$

De onde podemos ver claramente que, nesse caso particular simples, pode-se obter $\Phi \propto \ln (r), \operatorname{com} V(\Phi) \propto e^{2 \gamma \Phi}, \gamma$ uma constante, resultado tido como viável em cenários de formação de estruturas [?] além de modelos de quintessência [?].

\section{Considerações sobre os modelos com campos escalares}

Os dois primeiros modelos apresentados, são ancorados fortemente nos trabalhos de [?] e [?]. O primeiro deles, muito mais um toy-model, busca apenas uma introdução sucinta ao assunto, sem grandes pretensões no que tange a resultados, sejam eles quantitativos ou qualitativos, embora algumas características desejáveis já apareçam mesmo diante dessa abordagem simplista. O problema mais evidente, claro, reside no fato de que essas conclusões não são exatamente confiáveis, já que a análise despreza a interação gravitacional. Isso por um lado é bom, porque acaba resultando em um comportamento do tipo plano para as curvas de rotação em longas distâncias (o que é compensado por um campo que se estende até o infinito, não decaindo suficientemente rápido), mas deixa sempre a dúvida sobre de que maneira em uma situação mais realista o campo gravitacional poderia afetar esse resultado, por exemplo diminuindo a região sobre a qual o campo se estende.

O segundo deles opta por uma maneira fenomenológica de resolver o problema. Dadas as condições observadas para as curvas de rotação de galáxias, como adequar o campo a esse cenário. O resultado encontrado é, a primeira vista, atraente, sobretudo por ser inteiramente analítico. Os pontos fracos do método entretanto existem e alguns são graves, porque implicam por exemplo na necessidade de um potencial negativo para o campo, o que não é desejável, soando no mínimo muito artificial pela alta instabilidade. Além disso, alguns outros problemas como a não redescoberta do potencial Newtoniano no limite de campos fracos, ou impossibilidade de reobtenção da métrica de Minkowski para valores grandes de r, são também bastante incômodos, o que acaba justificando um ligeiro desvio desse tipo de abordagem ${ }^{3}$.

\subsubsection{Modelo Arbey et al [?], [?]}

Ressaltadas as primeiras dificuldades enfrentadas pelos modelos discutidos na seção anterior, passemos agora à apresentação de um trabalho que tem como um

\footnotetext{
${ }^{3} \mathrm{~A}$ referência [?] discute mais alguns problemas com o modelo
} 
de seus pontos de partida a solução de um dos problemas mais graves de [?], a necessidade de um potencial estritamente negativo para o campo.

\subsubsection{Considerações gerais}

O modelo apresentado agora se baseia novamente em um campo escalar com tensor de momento e energia dado por (4.40). Nesse caso, se supuséssemos uma métrica plana e um campo homogêneo, a densidade de energia poderia ser escrita como:

$$
\rho=T_{0}^{0}=\frac{\dot{\Phi}^{2}}{2}+V(\Phi)
$$

enquanto a pressão, $T^{i}{ }_{j}=-g_{j}^{i} P$, seria dada por:

$$
P=\frac{\dot{\Phi}^{2}}{2}-V(\Phi)
$$

Conforme pode ser visto de (4.57) e (4.58), no caso em que temos um termo cinético desprezível com relação à contribuição do termo potencial, recaímos no caso de uma constante cosmológica pura (vide Cap. 2), com equação de estado na forma $p=-\rho$, o que estimula na literatura o emprego de um campo escalar muito mais na tentativa de explicar a energia escura do que a matéria escura.

\subsubsection{Como um campo escalar pode simular a matéria es- cura}

Para o estudo do limite de campos fracos na relatividade geral, costuma-se olhar para uma métrica na forma:

$$
g_{\mu \nu}=\eta_{\mu \nu}+h_{\mu \nu}
$$

onde $\eta_{\mu \nu}$ é a métrica usual de Minkowski $(1,-1,-1,-1)$ e $h_{\mu \nu}$ é uma pequena perturbação na mesma. Nas condições do gauge harmônico [?, ?], temos:

$$
\partial_{\alpha} h_{\mu}^{\alpha}-\frac{1}{2} \partial_{\mu} h_{\alpha}^{\alpha}=0
$$

Entretanto, da equação de Einstein:

$$
S_{\mu \nu}=T_{\mu \nu}-\frac{1}{2} g_{\mu \nu} T_{\alpha}^{\alpha},
$$

onde $T_{\mu \nu}$ é o tensor de momento e energia e $S_{\mu \nu}$ é a chamada fonte, $\frac{R_{\mu \nu}}{8 \pi G}$. As soluções das equações de Einstein para $T_{\mu \nu}$ são bem conhecidas e têm a forma:

$$
h_{\mu \nu}=-4 G \int \frac{S_{\mu \nu}\left(\overrightarrow{r^{\prime}}\right)}{\left|\vec{r}-\overrightarrow{r^{\prime}}\right|} d^{3} \overrightarrow{r^{\prime}} .
$$


Por outro lado, na aproximação quase-newtoniana, o potencial gravitacional nada mais é que $\frac{h_{00}}{2}$, ou seja, de acordo com (4.61), ele é gerado por $S_{00}$. Sendo assim, para o caso de matéria sem pressão $(p \approx 0)$, devemos ter $2 S_{00}=T_{0}^{0}$. A conclusão a que chegamos é que o campo escalar gera um potencial gravitacional que é idêntico ao de uma componente de matéria escura que tivesse uma densidade de energia igual a $S_{00}$. Isso motiva a identificação dessa última quantidade como uma espécie de densidade efetiva de energia. Para um campo escalar como o descrito por (4.40), somos motivados a escrever:

$$
\rho_{\text {eff }} \equiv 2 S_{00}=2\left(\frac{\partial \Phi}{\partial t}\right)^{2}-2 V,
$$

conforme pode ser facilmente observado de (4.40) e (4.60). Entretanto, já se mostrou que o potencial gravitacional observado em galáxias não é facilmente reproduzido se levarmos em conta apenas a população de bárions. Geralmente, acrescenta-se a esses últimos uma componente de matéria escura, responsável pela massa faltante necessária para explicar, por exemplo, curvas de rotação. Todavia, se de fato quisermos que o campo escalar simule os efeitos esperados de uma componente escura de uma maneira universal, eles devem também responder pelos halos de matéria escura que se supõe existirem em galáxias (notadamente as de baixa luminosidade), onde a quantidade de matéria ordinária é praticamente desprezível quando comparada com a de matéria escura. Nesse limite, a sua densidade efetiva $\left(S_{00}\right)$ deve responder basicamente por uma densidade de matéria escura fria, sendo então positiva. Acrescendo a esse fato a hipótese de um potencial gravitacional praticamente estático, o que é uma boa aproximação em muitos casos, chegaríamos a conclusão que deveríamos ter $\dot{\Phi}=\partial_{0} \Phi=0$. Esse resultado, aplicado em (4.62), resulta em $\rho_{\text {eff }}=-2 V(\Phi)$, o que novamente força a necessidade de um potencial negativo para o campo (o mesmo inquietante problema já enfrentado no artigo [?]). Como resolver então a questão já que, por outro lado, um campo estático com potencial $\mathrm{V}$ positivo leva a um potencial newtoniano positivo, acarretando em repulsão e diminuindo a atração entre as galáxias? Um possível caminho se escora na seguinte idéia: por hipótese, olhemos para um campo escalar que varia muito mais rápido do que o sistema em que ele se envolve. Por exemplo, no caso da nossa Via-Láctea, que completa uma revolução em torno de aproximadamente 200 milhões de anos, estaríamos supondo um campo que varia numa escala de tempo muito menor, de modo que seus efeitos seriam sentidos apenas numa média:

$$
\rho_{e f f}=2\left\langle\dot{\Phi}^{2}\right\rangle-2\langle V\rangle
$$

Nas palavras de [?]:

"O comportamento do campo se mostrará similar ao de um oscilador harmônico, sempre que a pulsação $\mathrm{m}$ for muito maior que o vetor de onda $\mathrm{k}$ da configuração auto-gravitante". 
Ou seja, teremos a condição:

$$
\frac{2 \pi}{m} \ll R,
$$

onde $R$ é um comprimento típico de variação de $\Phi$ (ou seja, um comprimento para o qual o campo varia consideravelmente). Para os halos de galáxias, esse comprimento é da ordem de alguns $k p c$.

Como $\Phi$ varia como $e^{-i m t}$, podemos relacionar as médias das energias cinética e potencial (para um potencial do tipo $\lambda \Phi^{2}$ ) como:

$$
\left\langle\frac{\dot{\Phi}^{2}}{2}\right\rangle=\langle V(\Phi)\rangle .
$$

De (4.64) e (4.63), vemos que a densidade efetiva pode induzir uma atração gravitacional positiva, ou seja:

$$
\rho_{\text {eff }}=2\langle V(\Phi)\rangle>0 .
$$

Dessa forma, permitir que o campo vibre rapidamente acaba levando a uma mudança global de sinal da densidade efetiva. Além disso, como resultado de (4.58) e (4.64) teremos uma pressão associada ao campo nula. E esse comportamento é desejável, já que, nesse limite, o fluido escalar consegue simular os efeitos da matéria escura fria (lembrando que a equação de estado nos dois casos se torna essencialmente a mesma).

Entretanto, essa solução para o problema não é única. Uma outra maneira de enxergarmos essa solução é olharmos para um campo que apresente alguma espécie de simetria, girando em alguma espécie de "espaço interno" . Isso seria bastante útil, porque permitiria olhar para configurações onde quem se mostra estático não é a média temporal do campo e sim ele próprio. Uma maneira que parece ser a mais simples de concretizarmos essa idéia consiste em um campo escalar (esfericamente simétrico) com uma fase uniforme, do tipo ${ }^{4}$ :

$$
\Phi=\frac{\sigma(r)}{\sqrt{2}} e^{-i \omega t} .
$$

Para um campo dessa natureza, a densidade efetiva assume a forma:

$$
\frac{\rho_{e f f}}{2}=S_{00}=2 \dot{\Phi}^{*} \dot{\Phi}-U(\Phi) .
$$

Por outro lado, a pressão do fluido escalar é dada por:

$$
P \approx \dot{\Phi}^{*} \dot{\Phi}-U(\Phi)
$$

\footnotetext{
${ }^{4} \mathrm{Na}$ realidade, o ansatz realizado em 4.66 é bem fundamentado. É possível mostrar [?] que, para um número fixo de partículas $\mathrm{N}$, todas as soluções esfericamente simétricas de energia mínima apresentam dependência temporal exatamente como a apresentada.
} 
no limite em que as derivadas espaciais do campo $\left(\partial_{i} \Phi\right)$ são desprezíveis (em uma aproximação grosseira, isso é conseguido se assumirmos que $R$ é bastante maior que $\frac{1}{\omega}$, ou seja, $\left.\frac{\partial \Phi}{\partial r} \approx \frac{\sigma}{R} \ll \frac{\partial \Phi}{\partial t} \approx \sigma \omega\right)$. Prosseguindo, sempre que tivermos $\omega \approx m$, a pressão será bastante pequena e reconstruímos assim o resultado já conhecido para matéria escura fria. Além disso, ainda teremos, de (4.67), uma densidade efetiva que não dependerá explicitamente do tempo:

$$
\rho_{e f f}=\omega^{2} \sigma^{2}(r) .
$$

Nesse ponto, podemos abrir parênteses e tecer algumas considerações a respeito de um problema bastante interessante e provavelmente ainda não discutido a contento. São freqüentes na literatura especulações a respeito da possibilidade de o campo escalar responder tanto pela matéria escura quanto pela energia escura, ou seja, sobre se um mesmo ente (no caso um campo escalar) poderia responder de maneira satisfatória pelas propriedades de expansão acelerada do universo e, ao mesmo tempo, pela massa faltante nas galáxias. Chama-se isso de "quartessência" e temos algumas tentativas na literatura de abordar o problema das componentes escuras sob essa ótica, como por exemplo em [?, ?, ?, ?, ?].

Um tipo bastante comum de modelo dessa natureza utiliza um fluido com uma equação de estado do tipo:

$$
p_{c}=-\frac{A}{\left(\rho_{c}\right)^{\alpha}},
$$

onde $A$ e $\alpha$ são constantes. A equação (4.68) descreve o chamado gás de Chaplygin generalizado [?, ?], bastante comum em modelos que tentam unificar matéria escura e energia escura. No caso em que $\alpha=1,(4.68)$ descreve o gás de Chaplygin em sua formulação mais convencional. Nesse caso, da equação de conservação $d E=-p d V \Rightarrow d\left(\rho a^{3}\right)=-p d\left(a^{3}\right)$, temos:

$$
\rho=\sqrt{A+\frac{B}{a^{6}}}=\sqrt{A+B(1+z)^{6}},
$$

onde B é uma constante de integração. Desse modo, o gás de Chaplygin se comporta como matéria sem pressão ("dust") para tempos antigos e como constante cosmológica para tempos recentes. Na realidade, o gás de Chaplygin pode ainda ser derivado a partir da Lagrangeana de Born-Infeld conforme será mencionado no Cap. 5.

Um problema enfrentado pelo gás de Chaplygin convencional diz respeito à velocidade do som das perturbações, $v_{c}=\sqrt{\frac{d p_{C}}{d \rho_{c}}}=\frac{\sqrt{A}}{\rho_{c}}$, que cresce $\propto t^{2}$ na era dominada por matéria, ficando da ordem da velocidade da luz no presente. Dessa forma, analisando inomogeneidades do ponto de vista clássico, o gás de Chaplygin tem características que lembram mais a matéria escura quente $(H D M)$ do que a fria $C D M$, o que acaba suscitando idéias (por enquanto fenomenológicas) de utilização de um $\alpha \neq 1$ em (4.68). 
No caso do gás de Chaplygin generalizado, a equação de estado para o gás é também freqüentemente escrita (lembrando que $p=\omega \rho$ ):

$$
\omega(a)=-\frac{\left|\omega_{0}\right|}{\left[\left|\omega_{0}\right|+\frac{1-\left|\omega_{0}\right|}{a^{3(1+\alpha)}}\right]},
$$

que interpola entre $\omega=0$ para tempos antigos $(a \ll 1)$ e $\omega=-1$ para tempos posteriores $(a \gg 1), \omega_{0}$ sendo a equação de estado atual. A constante $\alpha$ regula a transição entre as duas fases.

Voltando ao caso do campo escalar canônico, da equação (4.62), vemos que o efeito gravitacional necessário para manter as galáxias unidas só deve acontecer se tivermos:

$$
\dot{\Phi}^{2} \geq V(\Phi) .
$$

Por outro lado, para respondermos pela expansão do universo (por exemplo, se quisermos simular o efeito de uma constante cosmológica), o potencial $V(\Phi)$ deve obedecer, de (4.58):

$$
\frac{\dot{\Phi}^{2}}{2} \leq V(\Phi)
$$

E aí surge a pergunta: qual deve ser a equação de estado para o campo escalar para que isso ocorra? Para que ambas essas condições, (4.70) e (4.71), sejam satisfeitas, se supusermos uma equação de estado do tipo $p=\omega \rho$ para 0 campo escalar devemos ter, de (4.57) e (4.58):

$$
\omega=\frac{P}{\rho}=\frac{\frac{\dot{\Phi}^{2}}{2}-V(\Phi)}{\frac{\dot{\Phi}^{2}}{2}+V(\Phi)} .
$$

A combinação de (4.72) com (4.70) e (4.71), implica em um $\omega>-\frac{1}{3}$, o que impõe sérios limites a essa possibilidade, conforme visto no Capt. 2. Embora, claro, seja conveniente ressaltar que o campo não necessariamente precisa ter o mesmo comportamento qualitativo em cosmologia e nos halos.

\subsubsection{Discussões gerais}

Neste caso, diferentemente do que foi discutido na seção anterior, a métrica a ser utilizada

$$
d \tau^{2}=e^{2 u(r)} d t^{2}-e^{2 v(r)}\left(d r^{2}+r^{2} d \theta^{2}+r^{2} \operatorname{sen}^{2} \theta d \phi^{2}\right),
$$

conhecida pelo nome de métrica isotrópica, é ligeiramente diferente de uma métrica estática esfericamente simétrica (como em (4.50)):

$$
d s^{2}=e^{2 u(\rho)} d t^{2}-e^{2 \bar{v}(\rho)} d \rho^{2}+\rho^{2}\left(d \theta^{2}+\sin ^{2} \theta d \phi^{2}\right),
$$


podendo ser obtida da mesma a partir de uma mudança de coordenadas conveniente [?]:

$$
\begin{gathered}
\rho=r e^{v} \\
e^{-\bar{v}}=\frac{d \ln \rho}{d \ln r}=1+r \frac{d v}{d r}
\end{gathered}
$$

Embora seja de uso menos freqüente que a métrica esfericamente simétrica, as razões para tal mudança ficarão claras ao longo do texto, pois o emprego de (4.73) proporcionará uma considerável simplificação nos cálculos.

Utilizando o ansatz para o campo (4.66), podemos calcular a equação de Klein-Gordon para o campo $\Phi$, ou seja:

$$
\square \Phi+\frac{\partial U(\Phi)}{\partial \Phi}=0
$$

Obtemos nesse caso a seguinte expressão:

$$
e^{-2 v}\left[\sigma^{\prime \prime}+\left(u^{\prime}+v^{\prime}+\frac{2}{r}\right) \sigma^{\prime}\right]+\omega^{2} e^{-2 u} \sigma-U^{\prime}(\sigma)=0 .
$$

Com algum esforço algébrico, podemos ainda escrever as equações de Einstein para o campo $\Phi$. A componente $r r$ aparece apenas como um vínculo adicional para as equações:

$$
2 u^{\prime} v^{\prime}+v^{\prime 2}+\frac{4 v^{\prime}}{r}=-8 \pi G(W+V-U) .
$$

Por outro lado, encontramos para a componente $t t$ :

$$
2 v^{\prime \prime}+v^{\prime 2}+\frac{4 v^{\prime}}{r}=-8 \pi G e^{2 v}(W+V+U) .
$$

E para as componentes $\theta \theta$ ou $\phi \phi$ :

$$
u^{\prime \prime}+v^{\prime \prime}+u^{\prime 2}+\frac{1}{r}\left(u^{\prime}+v^{\prime}\right)=8 \pi G e^{2 v}(W-V-U),
$$

onde $\mathrm{W}$ e $\mathrm{V}$ respondem por : $W=e^{-2 u}\left(\frac{\omega^{2} \sigma^{2}}{2}\right)$ e $V=e^{-2 v}\left(\frac{\sigma^{\prime 2}}{2}\right)$.

A idéia agora, a exemplo do que foi apresentado em [?], consiste na possibilidade de, trabalhando algumas condições físicas do problema, fazermos restrições acerca dos coeficientes da métrica (4.73). Por exemplo, supondo as chamadas "estrelas de bósons" ${ }^{5}$ assintoticamente planas e com energia finita, podemos limitar o espectro de valores permitidos para os parâmetros $u, v$ e $\sigma$, bem como de suas derivadas. A primeira observação é que, a fim de que tenhamos um espaçotempo assintoticamente Minkowskiano, é necessário que os parâmetros $u$ e $v$ da

\footnotetext{
[?].

${ }^{5}$ Que é o nome pelo qual são conhecidas essas configurações para esse campo escalar complexo
} 
métrica se anulem suficientemente rápido para grandes valores de $r$. Além disso, outra hipótese de trabalho é que, olhando para as configurações do campo a uma distância suficientemente grande, devemos redescobrir algo como Schwarschild, ou seja, nesse limite, seria como se uma massa puntual $M$ estivesse gerando a atração gravitacional. A métrica de Schwarschild para as coordenadas isotrópicas tem a forma:

$$
e^{u}=\frac{r-a}{r+a} ; e^{v}=\left(\frac{r+a}{r}\right)^{2} ; a=\frac{G M}{2} .
$$

Se supusermos o potencial para o campo da forma mais simples e convencional que se pode imaginar, teremos algo do tipo:

$$
U(\Phi)=m^{2} \Phi^{*} \Phi
$$

Antes de prosseguir na análise, é interessante ver o que uma versão simplificada da equação de Klein-Gordon para o campo $\Phi$ pode nos revelar. Por exemplo, para $\mathrm{r}$ grande, podemos reescrever a equação (4.76) na forma:

$$
\sigma^{\prime \prime}+\frac{2}{r} \sigma^{\prime}+\left(w^{2}-m^{2}\right) \sigma=0
$$

Entretanto, (4.51) só tem solução física interessante no caso em que $m>\omega$. E a razão disso é simples. No caso contrário, a solução da equação apresenta uma componente oscilatória, algo como $\frac{1}{r} \sin \left[\left(\omega^{2}-m^{2}\right)^{\frac{1}{2}} r\right]$ (como também já havia sido visto em (4.47)), o que por sua vez acaba preenchendo o universo com uma quantidade infinita de energia. Isso ainda pode ser contornado, desde que, artificialmente, introduzamos algum mecanismo que "corte" o campo em algum lugar. Essa não será a abordagem mostrada, já que esse é exatamente o tipo de problema que foi debatido quando do caso do MCE.

É possível ainda melhorar bastante o aspecto das equações para o campo olhando para algumas simetrias da ação. Se fizermos uma mudança de escala, reescrevendo:

$$
\bar{\sigma}=\sqrt{2 \pi G} \sigma, \bar{r}=m r,
$$

encontraremos para as equações de Klein-Gordon (4.76) e de Einstein (4.78) e (4.79):

$$
\begin{array}{r}
e^{-2 v}\left[\frac{d^{2} \bar{\sigma}}{d \bar{r}^{2}}+\left(u^{\prime}+v^{\prime}+\frac{2}{\bar{r}}\right) \frac{d \bar{\sigma}}{d \bar{r}}\right]+\left(\frac{\omega^{2}}{m^{2}} e^{-2 u}-1\right) \bar{\sigma}=0 \\
2 \frac{d^{2} v}{d \bar{r}^{2}}+\left(\frac{d v}{d \bar{r}}\right)^{2}+\frac{4}{\bar{r}} \frac{d v}{d \bar{r}}=-2 e^{2 v}\left\{\left[e^{-2 u} \frac{\omega^{2}}{m^{2}}+1\right] \bar{\sigma}^{2}+e^{-2 v}\left(\frac{d \bar{\sigma}}{d \bar{r}}\right)^{2}\right\}
\end{array}
$$




$$
\frac{d^{2} u}{d \bar{r}^{2}}+\left(\frac{d u}{d \bar{r}}\right)^{2}+\frac{1}{\bar{r}}\left(\frac{d u}{d \bar{r}}+\frac{d v}{d \bar{r}}\right)=2 e^{2 v}\left\{\left[e^{-2 u} \frac{\omega^{2}}{m^{2}}-1\right] \bar{\sigma}^{2}-e^{-2 v}\left(\frac{d \bar{\sigma}}{d \bar{r}}\right)^{2}\right\}
$$

Olhando para (4.84), (4.85) e (4.86), parece evidentemente interessante tomarmos a seguinte definição:

$$
e^{-2 \bar{u}}=\frac{\omega^{2}}{m^{2}} e^{-2 u}
$$

já que, escrevendo essas equações de forma adimensional, vemos que a dependência com a massa $m$ da partícula do campo, a velocidade de rotação $\omega$ e o parâmetro $u$ apenas aparece sob essa combinação bastante particular: $\frac{\omega^{2}}{m^{2}} e^{2 u}$. Mais informação relevante pode ser obtida se olharmos para o valor dessa função para valores arbitrariamente grandes de r. Como temos interesse em redescobrirmos Minkowski neste caso (ou seja, u tenderá a 0), podemos concluir, de (4.87):

$$
e^{-\bar{u}(\infty)}=\frac{\omega}{m}
$$

O estudo das equações de Einstein, bem como da equação de Klein-Gordon para o campo, pode se simplificar ainda mais ao olharmos para as mesmas no limite newtoniano, em que $|u| \ll 1$ e $|v| \ll 1$. Acresce-se a isso, um outro passo importante, sugerido em [?], que é relacionado com propriedades dos coeficientes da métrica isotrópica. Um parâmetro importante nessa análise é definido por:

$$
\xi^{2} \equiv 1-\frac{\omega^{2}}{m^{2}}
$$

o qual será suposto muito pequeno daqui pra frente, $\xi \ll 1$, ou seja, $\omega$ e $m$ serão aproximadamente iguais, mas sempre com $\omega<m$. Nesse caso, olhando para a equação de Klein-Gordon, $\sigma$ é aproximadamente proporcional a :

$$
\sigma \approx e^{-\left(m^{2}-\omega^{2}\right)^{\frac{1}{2}} r}=e^{-\xi m r},
$$

o que implica que uma derivada em r nas equações carregará termos do tipo:

$$
\frac{d}{d r} \sim \xi m
$$

o que permite estimar:

$$
\frac{\sigma^{\prime}}{m \sigma}=O(\xi)
$$

De maneira análoga, podemos inferir: 


$$
\frac{V}{U} \sim \frac{\left(\frac{d}{d r} \sigma\right)^{2}}{m^{2} \sigma^{2}}=O\left(\xi^{2}\right) .
$$

E, sendo $\omega \approx m$, podemos também escrever:

$$
\frac{U-W}{U} \sim \frac{m^{2}-\omega^{2}}{m^{2}}=O\left(\xi^{2}\right)
$$

Combinando as equações de Einstein para o campo [?], podemos escrever:

$$
U^{\prime}-V^{\prime}-W^{\prime}=2\left[u^{\prime}+v^{\prime}+\frac{2}{r}\right] V+2 u^{\prime} W .
$$

Baseados em (4.89), vemos que o tamanho radial da estrela de bósons deve ser da ordem de $\frac{1}{\xi m}$. Podemos assim supor que, para a maior parte do limite de validade da solução, devemos ter:

$$
r \sim \frac{1}{\xi m}
$$

Sendo assim, se utilizarmos (4.90), (4.92), (4.93) e (4.95) em (4.94) e, lembrando que $|u| \ll 1$ e $|v| \ll 1$, podemos primeiramente inferir que $u^{\prime} W$ deve ser da mesma ordem de $V^{\prime}$ e $U^{\prime}-W^{\prime}$.

Por outro lado, já vimos de (4.92) que $U \sim W \sim \frac{V}{\xi^{2}}$. Logo, combinando isso com o resultado para $u^{\prime} W$, encontramos:

$$
u=O\left(\xi^{2}\right) .
$$

Da equação de Einstein (4.78), podemos estimar:

$$
v \xi^{2} m^{2} \sim G U \rightarrow v=O\left(\frac{G U}{\xi^{2} m^{2}}\right)
$$

enquanto de (4.79):

$$
u+v=O\left(\frac{G U}{m^{2}}\right) .
$$

Para que (4.96), (4.97) e (4.98) sejam coerentes, devemos ter:

$$
\begin{gathered}
v=O\left(\xi^{2}\right), \\
\frac{G U}{m^{2}}=O\left(\xi^{4}\right),
\end{gathered}
$$

o que produz, finalmente:

$$
u+v=O\left(\xi^{4}\right)
$$


Ou seja, para $\omega \approx m$, podemos utilizar $u$ aproximadamente igual a $-v$, o que simplifica sobremaneira os cálculos. Utilizando a aproximação (4.101) nas equações de Einstein e de Klein Gordon (4.78) e (4.51), ficamos com:

$$
\sigma^{\prime \prime}+\frac{2}{r} \sigma^{\prime}=\left(m^{2}-\omega^{2} e^{-2 u}\right) \sigma
$$

E também:

$$
u^{\prime \prime}+\frac{2}{r} u^{\prime}=4 \pi G m^{2} \sigma^{2}
$$

Ou, em termos de $\bar{u}$ e $\bar{\sigma}$

$$
\begin{gathered}
\bar{u}^{\prime \prime}+\frac{2}{r} \bar{u}^{\prime}=2 \bar{\sigma}^{2}, \\
\bar{\sigma}^{\prime \prime}+\frac{2}{r} \bar{\sigma}^{\prime}=2 \bar{u} \bar{\sigma},
\end{gathered}
$$

sendo que, em (4.103) utilizamos $e^{-2 \bar{u}} \approx 1-2 \bar{u}$. O sistema descrito por (4.102) e (4.103) apresenta ainda mais uma peculiaridade, que consiste no fato de ele ser invariante por uma transformação de escala na forma:

$$
\begin{aligned}
& \bar{u} \rightarrow k \bar{u} \\
& \bar{\sigma} \rightarrow k \bar{\sigma} \\
& \bar{r} \rightarrow k^{-\frac{1}{2}} \bar{r}
\end{aligned}
$$

característica esta que garante o caráter solitônico das soluções.

Nos gráficos apresentados a seguir, o sistema (4.102) e (4.103) foi normalizado mais uma vez, sendo escrito em sua forma final em termos de $S \equiv \frac{\bar{\sigma}(r)}{\bar{\sigma}(0)}$, de $u_{*} \equiv \frac{\bar{u}(r)}{\bar{\sigma}(0)}$ e de $x \equiv m r \sqrt{\bar{\sigma}(0)}$ :

$$
\begin{aligned}
u_{*}^{\prime \prime}+\frac{2}{x} u_{*}^{\prime} & =2 S^{2}, \\
S^{\prime \prime}+\frac{2}{x} S^{\prime} & =2 u_{*} S .
\end{aligned}
$$

Esse último sistema de equações tem propriedades bastante interessantes e é possível de ser resolvido via métodos numéricos. Para reproduzir os resultados encontrados no artigo [?], programou-se um código em C (Runge-Kutta de quarta ordem, com passo adaptativo). As equações foram então integradas para algumas condições iniciais particulares. A grande chave do problema consiste na determinação dessas condições. A escolha delas se baseava em um critério bastante simples. Elas eram tais que conduziriam no "infinito" a soluções em que $\bar{u}$ 
deveria convergir e $v$ e $\bar{\sigma}$ deveriam ir suavemente decrescendo para zero. No artigo original [?], o trabalho de determinação dessas condições era feito através do chamado "shooting method", uma técnica de integração numérica que consiste em casarmos as condições existentes em dois extremos da integração [?]. No código que foi feito, optamos por um método alternativo e conseguimos reproduzir boa parte dos resultados. A saída empregada foi a seguinte: correríamos um grande "array" de condições iniciais e veríamos quais delas proporcionariam soluções que pudessem ser integradas até o maior raio possível antes de saírem do limite de validade da aproximação newtoniana, ou seja, que demorassem mais para atingir o regime não-linear e proporcionassem configurações mais estáveis para o campo, como pode ser visto em (4.2). Como poderá ser visto a seguir, os resultados coincidem com os encontrados no artigo original, mostrando que a solução por eles encontrada, além de ser aquela que satisfaz as condições de contorno é também a que permanece por mais tempo dentro dos limites de validade da aproximação newtoniana.

Posteriormente, foram feitos gráficos das curvas de rotação (velocidade circular como função do raio) de partículas-teste nesse espaço-tempo preenchido por um campo escalar, com as condições iniciais obtidas pelo método descrito anteriormente. A identificação das funções da métrica com essa velocidade é feita de maneira quase trivial. Para uma métrica $g_{\mu \nu}$ que é aproximadamente Minkowski ( $g_{\mu \nu}=\eta_{\mu \nu}+h_{\mu \nu}$, onde os $h_{\mu \nu}$ são pequenas perturbações), como é o caso suposto aqui, é possível mostrar que o elemento $h_{00}$ está intrinsecamente relacionado com o potencial gravitacional gerador desta perturbação, da seguinte maneira [?]:

$$
h_{00}=2 \Psi_{\text {grav }} .
$$

Para o nosso caso, como $g_{00}=e^{2 u} \approx 1+2 u$, isso se traduz na condição:

$$
u=\Psi_{\text {grav }} .
$$

Por outro lado, das leis de Newton, sabemos que a velocidade circular de rotação pode ser obtida da seguinte expressão:

$$
v_{c}^{2}=r \frac{d \Psi_{\text {grav }}}{d r}=r|\vec{F}|=r u^{\prime}
$$

logo:

$$
v_{c}=\sqrt{r u^{\prime}}
$$

Os gráficos a seguir resumem os resultados encontrados, apresentando as condições iniciais interessantes para $u_{*}(x)$ bem como as curvas de rotação derivadas a partir desses valores. 


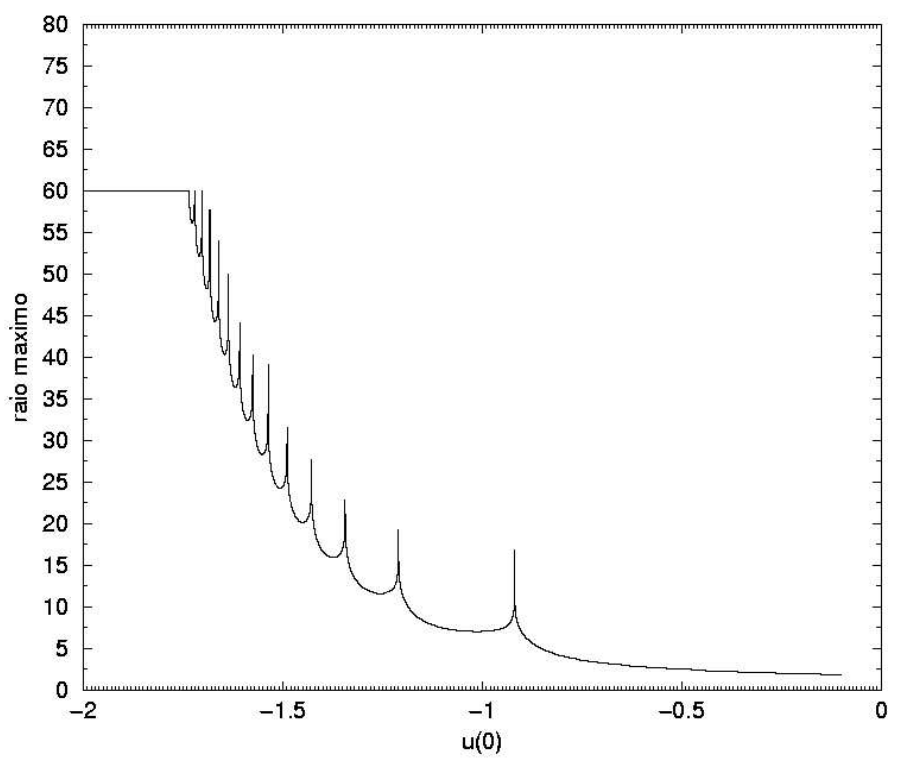

Figura 4.2: Espectro das possíveis condições iniciais que levam a um decaimento da solução para a métrica $u(x)$ no infinito. Os picos se referem à condições iniciais que levam à soluções com comportamento assintótico decrescente para as curvas de rotação.

A expressão (4.106) aparece plotada a seguir para algumas condições iniciais encontradas no gráfico (4.2) (ou seja, para condições que obedecem os critérios de convergência já citados anteriormente):
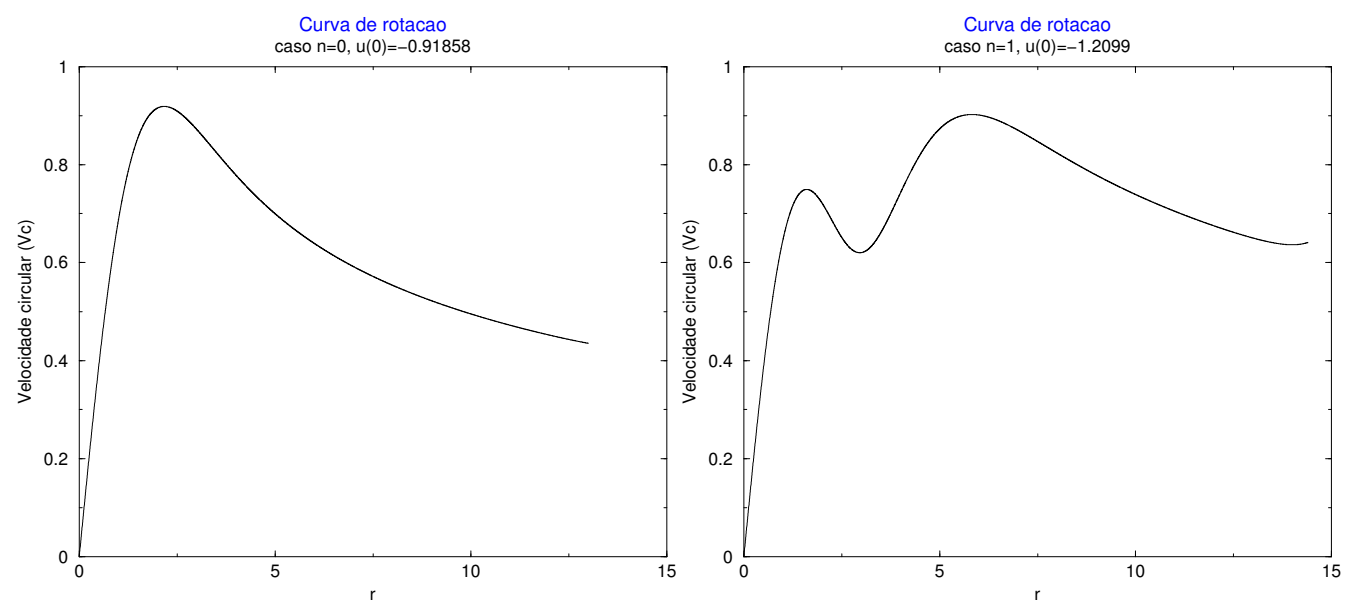

Figura 4.3: Curvas de rotação para os autovalores $\mathrm{n}=0$ e $\mathrm{n}=1$. 

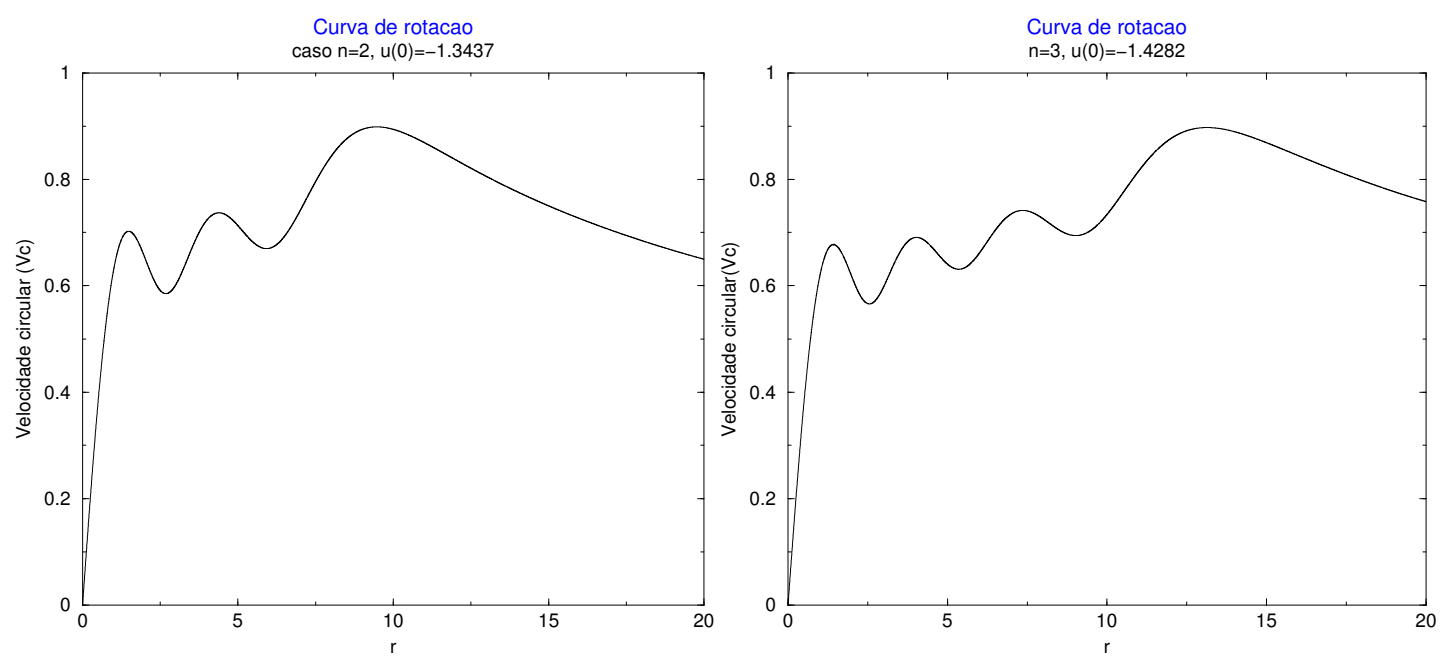

Figura 4.4: Curvas de rotação para os autovalores n=2 e n=3.
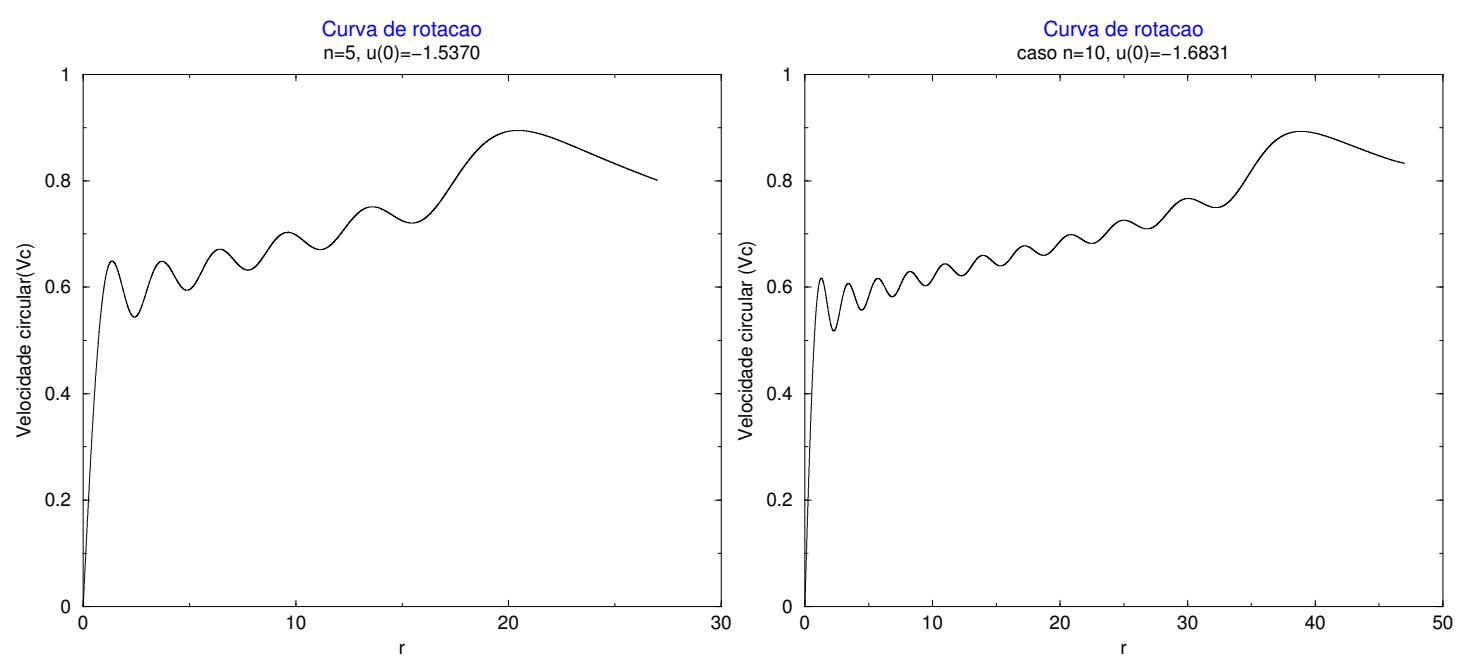

Figura 4.5: Curvas de rotação para os autovalores autovalores $n=5$ e $n=10$. 


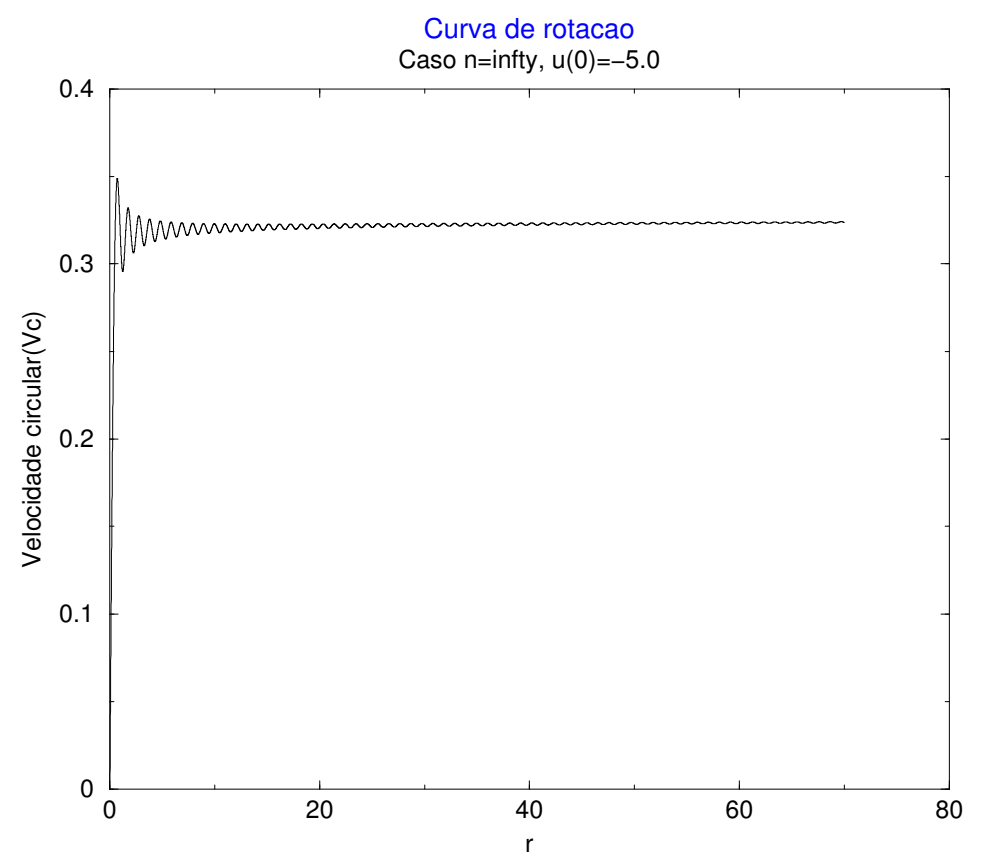

Figura 4.6: Curva de rotação para o autovalor $\mathrm{n}=\infty$.

O resultado apresentado aqui é particularmente interessante, por várias razões. Uma delas é a de que, nesse limite newtoniano, as equações para a evolução do campo apresentam um espectro discreto de condições iniciais, com as propriedades de convergência já discutidas. As configurações encontradas partindo da evolução dessas condições iniciais podem então ser interpretadas como autoestados de energia do sistema, sendo que o estado de mínima energia é caracterizado pela ausência de nós (estados onde $\sigma(r)=0$ ), enquanto cada estado excitado terá $\mathrm{n}$ nós, conforme pode ser visto nas figuras anteriores.

Nos capítulo seguintes, quando analisarmos o campo de Born-Infeld, discutiremos uma possível razão para o aparecimento desse espectro discreto e dessas soluções. 


\section{Capítulo 5}

\section{Campo escalar de Born-Infeld}

O trabalho que passa a ser agora apresentado foi fortemente inspirado pelos artigos do capítulo anterior. Também estudamos configurações estáticas e esfericamente simétricas das equações de campo de Einstein, apenas com a diferença de que, agora, elas tinham como termo de fonte um caso particular dos chamados modelos de k-essência. Tentamos analisar quais seriam as configurações que obteríamos para um campo com uma lagrangeana ligeiramente diferente da canônica. A lagrangeana utilizada tem motivação na teoria de cordas [?], e é uma espécie de generalização da lagrangeana para uma partícula relativística [?], só que, neste caso, é aplicada no contexto de campos. A chamada lagrangeana de Born-Infeld tem a seguinte expressão:

$$
\mathcal{L}=-U(\Phi) \sqrt{1-g_{\alpha \beta} \partial^{\alpha} \Phi \partial^{\beta} \Phi}
$$

onde $U(\Phi)$ é o potencial do campo.

Como já visto no capítulo anterior, podemos extrair o tensor de momentoenergia para essa lagrangeana (4.39):

$$
T_{\mu \nu}=U(\Phi) \sqrt{1-\partial_{\mu} \Phi \partial^{\mu} \Phi} g_{\mu \nu}+\frac{U(\Phi)\left(\partial_{\nu} \Phi \partial_{\mu} \Phi\right)}{\sqrt{1-g_{\alpha \beta} \partial^{\alpha} \Phi \partial^{\beta} \Phi}}
$$

Tomando $u^{\mu}=\frac{\partial^{\mu} \Phi}{\sqrt{\partial^{\alpha} \Phi \partial_{\alpha} \Phi}}$, podemos redescobrir em (5.2) a forma convencional do tensor de momento e energia para um fluido perfeito:

$$
T_{\mu \nu}=(\rho+p) u_{\mu} u_{\nu}-p g_{\mu \nu}
$$

onde

$$
\begin{aligned}
\rho & =\frac{V}{\sqrt{1-\partial^{\alpha} \Phi \partial_{\alpha} \Phi}}, \\
p & =-V \sqrt{1-\partial^{\alpha} \Phi \partial_{\alpha} \Phi} \\
\frac{p}{\rho} & =-1+\partial^{\alpha} \Phi \partial_{\alpha} \Phi=-\frac{p^{2}}{V^{2}} \Rightarrow p=-\frac{V^{2}}{\rho}
\end{aligned}
$$


exatamente a forma proposta por (4.68), $\operatorname{com} A=V^{2}$, o que faz a conexão desse modelo com o do gás de Chaplygin.

No nosso caso, a fim de buscarmos uma correlação com os resultados obtidos com uma lagrangeana canônica [?], optamos por um caminho ligeiramente diferente, uma tentativa de extrapolação. Tentamos trabalhar com uma lagrangeana de Born-Infeld generalizada para acomodar um campo complexo. Nesse caso, obtivemos as seguintes expressões para a lagrangeana e para o tensor de momentoenergia:

$$
\begin{gathered}
\mathcal{L}=-U\left(\Phi, \Phi^{*}\right) \sqrt{1-g_{\alpha \beta} \partial^{\alpha} \Phi^{*} \partial^{\beta} \Phi} \\
T_{\mu \nu}=U\left(\Phi, \Phi^{*}\right)\left[\frac{1}{2}\left(\frac{\partial_{\mu} \Phi^{*} \partial_{\nu} \Phi+\partial_{\mu} \Phi \partial_{\nu} \Phi^{*}}{\sqrt{1-g_{\alpha \beta} \partial^{\alpha} \Phi^{*} \partial^{\beta} \Phi}}\right)+\sqrt{1-\partial_{\alpha} \Phi^{*} \partial^{\alpha} \Phi} g_{\mu \nu}\right] .
\end{gathered}
$$

De posse desses resultados, podemos escrever a equação de Euler-Lagrange para o campo, a qual nos fornece ${ }^{1}$ :

$$
\begin{aligned}
& \square \Phi+\frac{1}{2} g_{\gamma \delta} \frac{\nabla_{\mu}\left(\partial^{\gamma} \Phi^{*} \partial^{\delta} \Phi\right) \partial^{\mu} \Phi}{1-g_{\alpha \beta} \partial^{\alpha} \Phi^{*} \partial^{\beta} \Phi}-2 \ln U\left(\Phi, \Phi^{*}\right), \Phi^{*}+ \\
& +\frac{1}{U\left(\Phi, \Phi^{*}\right)}\left[g_{\alpha \beta} \partial^{\beta} \Phi \frac{\partial U\left(\Phi, \Phi^{*}\right)}{\partial \Phi^{*}}\left(\partial^{\alpha} \Phi^{*}-\partial^{\alpha} \Phi\right)\right]=0
\end{aligned}
$$

As equações foram escritas explicitamente em termos de uma métrica esfericamente simétrica e de outra, isotrópica, como foi feito no capítulo anterior. As equações explícitas para a métrica esfericamente simétrica estão em termos de:

$$
d s^{2}=e^{2 \alpha(\rho)} d t^{2}-e^{2 \lambda(\rho)} d \rho^{2}-\rho^{2} d \Omega^{2} .
$$

Nesse caso, encontramos as seguintes equações de Einstein:

$$
\begin{gathered}
\frac{2 \lambda^{\prime}}{x}-\frac{1}{x^{2}}+\frac{e^{2 \lambda(x)}}{x^{2}}=\frac{1}{\omega^{2}}\left(\frac{8 \pi G(1+2 V) e^{2 \lambda(x)} U\left(\Phi, \Phi^{*}\right)}{\sqrt{(1+2 V-2 W)}}\right), \\
\frac{2 \alpha^{\prime}}{x}+\frac{1}{x^{2}}-\frac{e^{2 \lambda(x)}}{x^{2}}=\frac{1}{\omega^{2}}\left(\frac{8 \pi G(-1+2 W) e^{2 \lambda(x)} U\left(\Phi, \Phi^{*}\right)}{\sqrt{(1+2 V-2 W)}}\right), \\
\alpha^{\prime \prime}-\lambda^{\prime} \alpha^{\prime}+\alpha^{\prime 2}+\frac{\alpha^{\prime}-\lambda^{\prime}}{x}=-\frac{1}{\omega^{2}}\left(8 \pi G e^{2 \lambda(x)} U\left(\Phi, \Phi^{*}\right) \sqrt{(1+2 V-2 W)}\right) .
\end{gathered}
$$

\footnotetext{
${ }^{1}$ Sendo que a equação para $\Phi^{*}$ pode ser obtida de maneira análoga
} 
A equação para o campo pode ser obtida mais facilmente da analogia das componentes do campo com as de um fluido $\left(T^{t}{ }_{t}=\rho, T_{r}^{r}=-p_{r}, T^{\theta}{ }_{\theta}=-p_{\theta}\right.$, $\left.T_{\phi}^{\phi}=-p_{\phi}\right)$. Nesse caso, temos a chamada equação de Tolman-OppenheimerVolkov que, generalizada para incluir a anisotropia das pressões, fornece:

$$
\frac{d p_{r}}{d r}=-\left(\rho+p_{r}\right) 2 \alpha^{\prime}+\frac{2}{r}\left(p_{t}-p_{r}\right) .
$$

De (5.12) ou via conservação do tensor de energia-momento (5.7), já que as equações são equivalentes, obtemos a seguinte equação para o campo de BornInfeld:

$$
\begin{gathered}
\tilde{\sigma}^{\prime \prime}=\frac{\tilde{\sigma}^{\prime}}{2 V}\left[\frac{1+2 V-2 W}{1-2 W}\left((1-2 W) \frac{d}{d \tilde{\sigma}^{\prime}} \ln U\left(\Phi, \Phi^{*}\right)-2 V\left(\frac{2}{x}+\alpha^{\prime}\right)\right)\right]+ \\
+\left[\frac{1+2 V-2 W}{1-2 W}\right] \frac{W \tilde{\sigma}^{\prime}}{V}\left(\frac{2 \tilde{\sigma}^{\prime}}{\tilde{\sigma}}-\alpha^{\prime}\right)+\frac{\tilde{\sigma}^{\prime}}{2 V}\left(2 V \lambda^{\prime}-2 W \alpha^{\prime}+\frac{2 W \tilde{\sigma}^{\prime}}{\tilde{\sigma}}\right),
\end{gathered}
$$

para um campo suposto na forma: $\Phi(\rho, t)=\sigma(\rho) e^{-i \omega t}$, com $W=\frac{1}{2} e^{-2 \alpha(x)} \tilde{\sigma}^{2}$, $V=\frac{1}{2} e^{-2 \lambda(x)} \tilde{\sigma}^{\prime 2}, x=\omega \rho$ e $\tilde{\sigma}=\omega \sigma$.

Podemos ainda escrever (5.13) em uma forma mais explícita e mais sugestiva:

$$
\begin{aligned}
\tilde{\sigma}^{\prime \prime}+\frac{2 \tilde{\sigma}^{\prime}}{x}+ & e^{2 \lambda(x)-2 \alpha(x)} \tilde{\sigma}=-\frac{\tilde{\sigma}^{\prime} \alpha^{\prime}\left(e^{-2 \lambda(x)} \tilde{\sigma}^{\prime 2}-e^{-2 \alpha(x)} \tilde{\sigma}^{2}\right)}{1-e^{-2 \alpha(x)} \tilde{\sigma}^{2}}+ \\
& -\left(\lambda^{\prime}-\alpha^{\prime}\right) \tilde{\sigma}^{\prime}-\frac{2 \tilde{\sigma}^{\prime 2}}{1-e^{-2 \alpha(x)} \tilde{\sigma}^{2}}\left(e^{-2 \lambda(x)} \frac{\tilde{\sigma}^{\prime}}{x}+e^{-2 \alpha(x)} \tilde{\sigma}\right)+ \\
& +\left(1+e^{-2 \lambda(x)} \tilde{\sigma}^{\prime 2}-e^{-2 \alpha(x)} \tilde{\sigma}^{2}\right) e^{2 \lambda(x)} \frac{d}{d \tilde{\sigma}^{\prime}} \ln U\left(\Phi, \Phi^{*}\right) .
\end{aligned}
$$

Já para uma métrica isotrópica, como em (4.73):

$$
d s^{2}=e^{2 u(r)} d t^{2}-e^{2 v(r)}\left(d r^{2}+r^{2} d \theta+r^{2} \operatorname{sen}^{2} \theta d \phi^{2}\right),
$$

obtemos, para as equações de Einstein (5.15), (5.16), (5.17) e para a equação do campo de Born-Infeld (5.18) (novamente suposto na forma: $\Phi(r, t)=\sigma(r) e^{-i \omega t}$ ), respectivamente:

$$
\begin{gathered}
2 v^{\prime \prime}+v^{\prime 2}+\frac{4 v^{\prime}}{x}=-\frac{8 \pi G}{\omega^{2}} \frac{\left(1+e^{-2 v(x)} \widetilde{\sigma}^{\prime 2}\right) e^{2 v(x)}}{\sqrt{\left(1+e^{-2 v(x)} \widetilde{\sigma}^{\prime 2}-e^{-2 u(x)} \widetilde{\sigma}^{2}\right)}} U\left(\Phi, \Phi^{*}\right), \\
2 u^{\prime} v^{\prime}+\frac{2}{x}\left(u^{\prime}+v^{\prime}\right)+v^{\prime 2}=-\frac{8 \pi G}{\omega^{2}} \frac{\left(1+e^{-2 u(x)} \widetilde{\sigma}^{2}\right) e^{2 v(x)}}{\sqrt{\left(1+e^{-2 v(x)} \widetilde{\sigma}^{\prime 2}-e^{-2 u(x)} \widetilde{\sigma}^{2}\right)}} U\left(\Phi, \Phi^{*}\right),
\end{gathered}
$$




$$
\begin{gathered}
v^{\prime \prime}+u^{\prime \prime}+u^{\prime 2}+\left(u^{\prime}+v^{\prime}\right) \frac{1}{x}=-\frac{8 \pi G}{\omega^{2}} \sqrt{1+e^{-2 v(x) \widetilde{\sigma}^{\prime 2}-e^{-2 u(x)} \widetilde{\sigma}^{2}} e^{2 v(x)} U\left(\Phi, \Phi^{*}\right),} \\
\widetilde{\sigma}^{\prime \prime}=v^{\prime} \widetilde{\sigma}^{\prime}+e^{-2 u(x)+2 v(x)} \widetilde{\sigma}+\frac{1}{1-e^{-2 u(x)} \widetilde{\sigma}^{2}}\left(u^{\prime} e^{-2 u(x)} \widetilde{\sigma}^{2} \widetilde{\sigma}^{\prime}\right)+ \\
+\left(1+e^{-2 v(x)} \widetilde{\sigma}^{\prime 2}-e^{-2 u(x)} \widetilde{\sigma}^{2}\right)\left[e^{2 v(x)} \frac{d}{d \tilde{\sigma}^{\prime}} \ln U\left(\Phi, \Phi^{*}\right)\right]+ \\
-\left(1+e^{-2 v(x)} \widetilde{\sigma}^{\prime 2}-e^{-2 u(x)} \widetilde{\sigma}^{2}\right)\left[\frac{\widetilde{\sigma}^{\prime}\left(2 v^{\prime}+\frac{2}{x}+u^{\prime}\right)-2 e^{-2 u(x)+2 v(x)} \widetilde{\sigma}}{1-e^{-2 u(x)} \widetilde{\sigma}^{2}}\right]
\end{gathered}
$$

onde novamente foram feitas as seguintes mudanças de variáveis ${ }^{2}$ :

$$
\begin{aligned}
& \widetilde{\sigma}=\omega \sigma, \\
& x=\omega r .
\end{aligned}
$$

Escritas as equações, nosso primeiro passo foi tentar solucionar o mais simples dos problemas, que seria resolvê-las no caso em que o potencial era constante e em um background de Minkowski, assim como foi feito no caso mostrado no capítulo anterior [?]. Nesse caso, a equação a ser resolvida é a $(5.18)^{3}$ que, simplificada $\left(U\left(\Phi, \Phi^{*}\right)=\right.$ constante, $\left.u=v=0\right)$, assume a seguinte forma:

$$
\widetilde{\sigma}^{\prime \prime}+\frac{2}{x} \widetilde{\sigma}^{\prime}+\widetilde{\sigma}=-\frac{2 \widetilde{\sigma}^{\prime 2}}{1-\widetilde{\sigma}^{2}}\left(\widetilde{\sigma}+\frac{\widetilde{\sigma}^{\prime}}{x}\right)
$$

Embora ainda se trate de uma equação altamente não-linear, o primeiro detalhe que fomos capazes de perceber foi a existência de uma solução analítica bastante simples para (5.19), que aparece na forma:

$$
\widetilde{\sigma}(x)= \pm \cosh x
$$

$\operatorname{com} \widetilde{\sigma}(0)=1$ e $\widetilde{\sigma}^{\prime}(0)=0$. Na realidade, essa solução é ainda mais poderosa, pois aparece mesmo em casos em que o potencial não é constante, como pode ser

\footnotetext{
${ }^{2}$ Apenas por completeza, a equação (5.18) também pode ser obtida via analogia com o caso de fluidos e nesse caso:

$$
\frac{d p_{r}}{d r}=-\left(2 v^{\prime}+\frac{2}{r}\right)\left(p_{r}-p_{t}\right)+\left(p_{r}+\rho\right) u^{\prime}
$$

${ }^{3} \mathrm{Na}$ realidade, as equações (5.18) e (5.14) são equivalentes nesse limite, já que as métricas para ambas coincidem nesse caso.
} 
visto por simples substituição da mesma em (5.18) (isso, claro, ainda no limite em que $u=v=0$ ).

Entretanto, essa solução aparentemente promissora tem seus problemas. O primeiro deles é o fato de, sendo (5.19) não linear, (5.20) não constitui um conjunto completo de soluções. O segundo, ainda mais grave, é que (5.20) é uma solução que descreve um ramo não físico da equação (5.19). Isso é relativamente fácil de ser percebido, bastando inserir essa solução na lagrangeana (5.1). Nesse caso, a lagrangeana se torna identicamente nula, reiterando nossa afirmação.

Dada a impossibilidade de trabalharmos com a solução exata encontrada, partimos para um tratamento numérico. Com uma rotina de Runge-Kutta de quarta ordem por nós desenvolvida, realizamos a integração numérica da equação (5.19). Um resultado representativo do que foi encontrado aparece em (5.1), que demonstra claramente um caráter oscilatório (em torno da origem) para $\sigma(x)$.
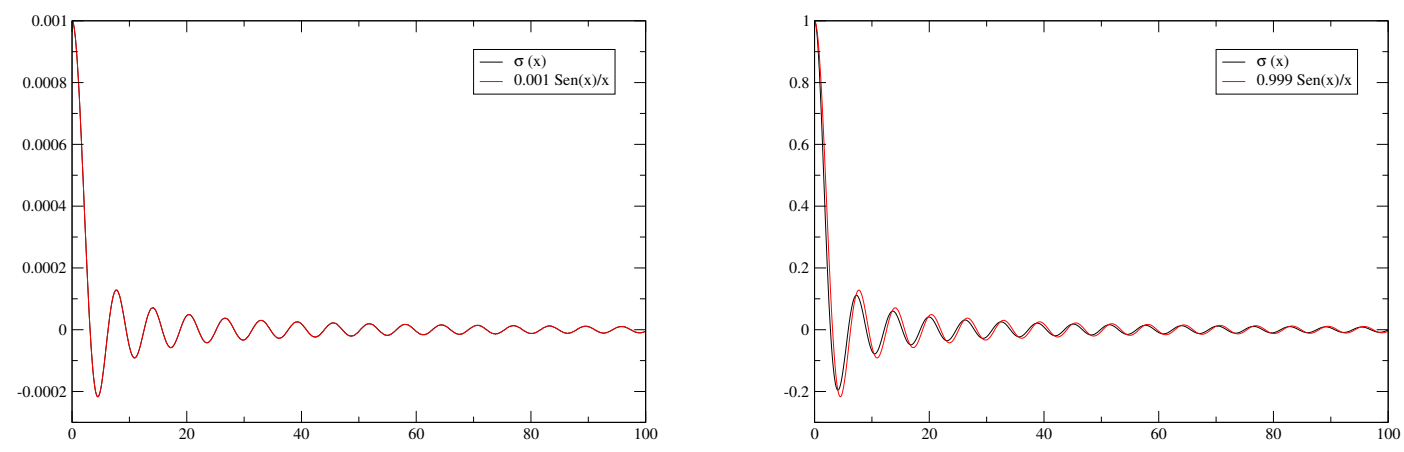

Figura 5.1: Gráfico de $\sigma(x)$ no caso de um potencial $U\left(\Phi, \Phi^{*}\right)$ constante para duas condições iniciais representativas do comportamento da função. Uma para uma condição inicial próxima de 0, outra próxima de 1. Em ambos os casos, $\tilde{\sigma}^{\prime}(0)$ foi suposto nulo. Em cada gráfico, plotouse também uma curva do tipo $\sigma(0) \frac{\sin (x)}{x}$, que averiguamos aproximar com relativa precisão a solução exata.

A constatação de que uma solução do tipo $\sigma(0) \frac{\sin (x)}{x}$ aproxima bem a solução exata pode ser constatada da inspeção da equação (5.19). Sendo pequeno o valor da derivada $\widetilde{\sigma}^{\prime}$, o lado esquerdo de (5.19) é praticamente desprezível. Nesse caso,a solução da equação é do tipo $\widetilde{\sigma}(x)=\frac{A \sin x}{x}+\frac{B \cos x}{x}$. Sendo a condição inicial para a derivada do campo nula na origem (para evitar singularidades), teremos $B=0$, ficando $\operatorname{com} \tilde{\sigma}(x)=\frac{\sigma_{0} \sin x}{x}$.

Também foi feito um gráfico para a densidade de energia do campo $\left(T_{0}{ }^{0} \equiv \rho\right)$ e para as pressões, radial $\left(-T_{1}{ }^{1} \equiv p_{r}\right)$ e tangencial $\left(-T_{2}{ }^{2}=-T_{3}{ }^{3} \equiv p_{t}\right)$. 

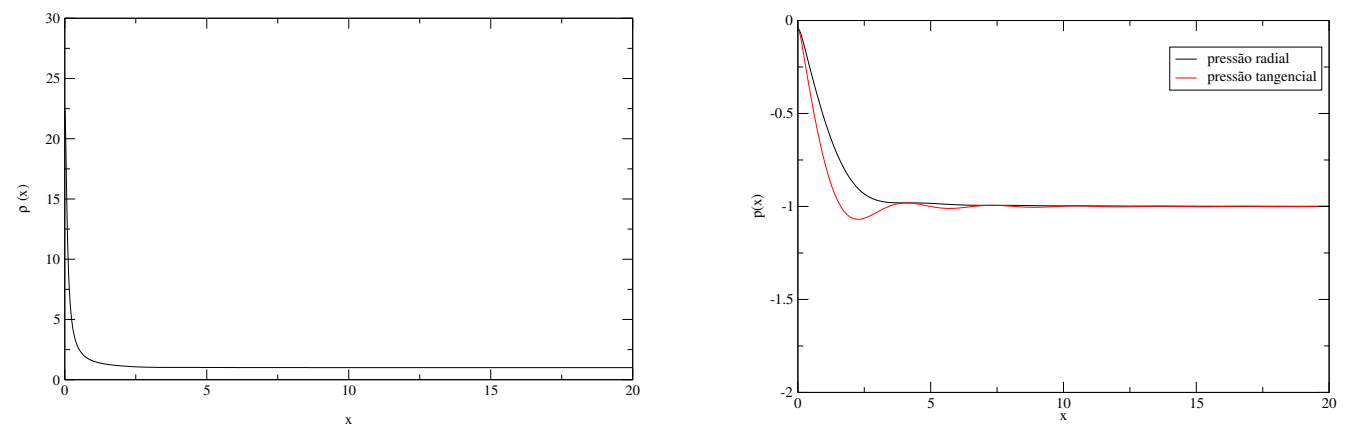

Figura 5.2: Gráficos para a densidade de energia e pressões do campo. No caso apresentado, foi utilizado um $\widetilde{\sigma}(0)=0.999, \tilde{\sigma}^{\prime}(0)=0$ e o potencial $U\left(\Phi, \Phi^{*}\right)$, suposto constante, foi normalizado para 1.

Da análise dos gráficos, pudemos observar que o campo apresenta um regime fortemente oscilatório, que se mostra mais acentuado quando as condições iniciais para o campo $(\widetilde{\sigma}(0))$ estão mais próximas de 1 (nos demais casos as oscilações são bem menores). Em todos os casos, entretanto, as componentes de pressão para grandes distâncias se mostram relativamente grandes, da mesma ordem de grandeza da densidade de energia, o que talvez não seja tão interessante quando a idéia é modelar matéria escura. Também é importante ressaltar que, para grandes distâncias, o campo tende a oscilar com amplitude cada vez menor em torno de zero. O curioso desse comportamento é que, mesmo nesse caso, tanto a densidade de energia quanto as pressões do campo não são nulas, como pode ser observado na figura (5.2). Por sua vez, esse suposto problema parece, à primeira vista, ligeiramente simples de ser contornado. Bastaria que descontássemos da solução final essa espécie de contribuição de fundo, ou seja, os valores da densidade e das pressões para quando $x \rightarrow \infty$. No caso de um background de Minkowski, isso se mostra trivial, já que a solução final para $\widetilde{\sigma}(x)$ simplesmente oscila (com pequena amplitude) em torno de zero e, na aproximação até aqui adotada, não interfere na métrica. Por outro lado, em casos um pouco mais elaborados, como alguns que serão apresentados no capítulo seguinte, a situação é ligeiramente diferente e essa interpretação deixa de ser óbvia.

Embora essa primeira solução encontrada ainda não se mostrasse suficientemente interessante para nosso ideal, optamos por manipulá-la mais algum tempo. Descobrimos que, para valores iniciais de $\widetilde{\sigma}$ próximos de zero, a envoltória da curva em 5.1 é muito bem aproximada por uma lei de potência do tipo $\frac{1}{x}$. Na realidade, uma solução do tipo $\frac{\sin (x)}{x}$ descreve bastante bem a solução para o campo (5.1), o que sugere que o lado direito em (5.19) é praticamente desprezível nesses casos (ou seja, encontramos um resultado parecido com aquele já apresentado no capítulo anterior [?, ?]). Já com relação à densidade de energia, observamos que, para 
uma vasta gama de condições iniciais diferentes dentro do intervalo $0<\widetilde{\sigma}(0)<1$, o comportamento qualitativo encontrado é essencialmente o seguinte: o halo apresenta a região mais interna com uma densidade que varia muito lentamente com o raio, sendo aproximadamente constante até um $x$ da ordem de 0.1 , para depois apresentar um decaimento bastante acentuado (mais rápido quanto maior a condição inicial para o campo), conforme pode ser constatado na figura 5.3.

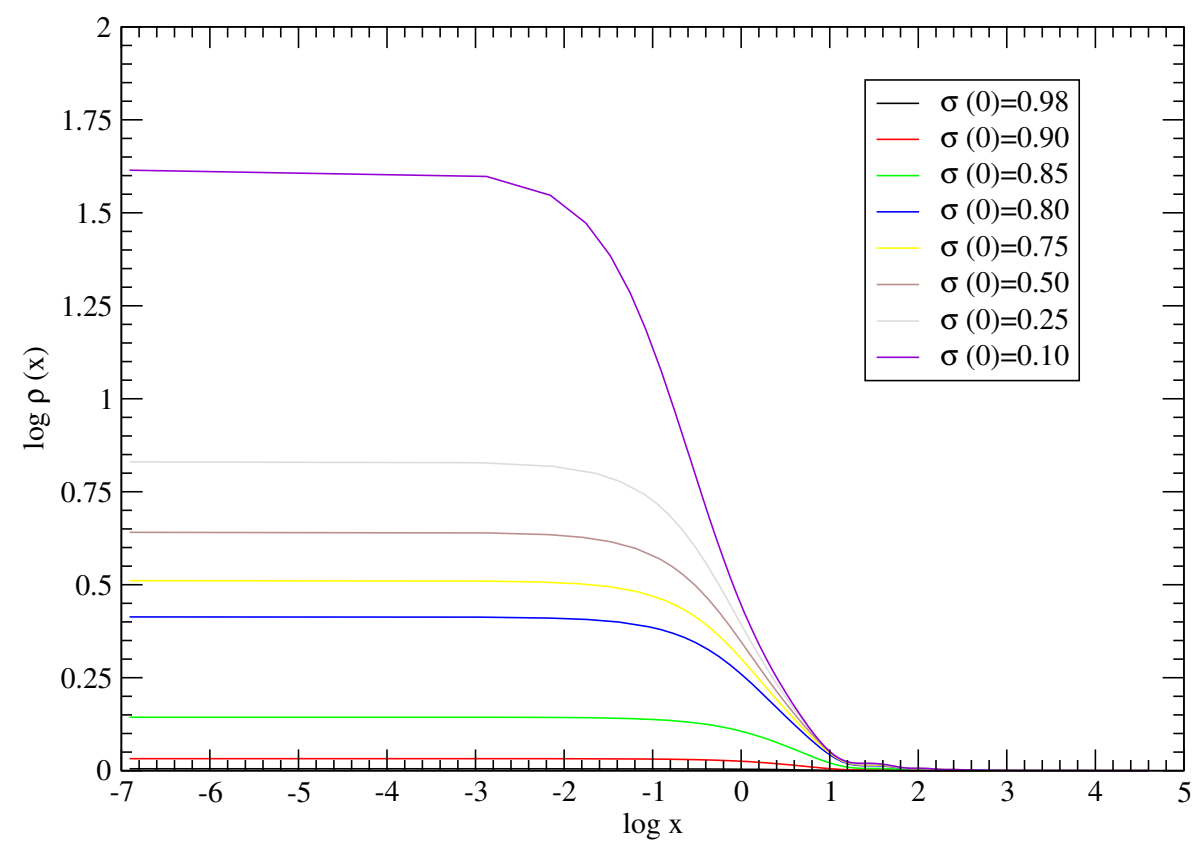

Figura 5.3: Gráfico log-log da densidade de energia para várias condições iniciais diferentes .

Em resumo, a solução que foi encontrada nesse primeiro caso ainda não é capaz de descrever de maneira satisfatória um halo de matéria escura. O decaimento da solução não é suficientemente rápido (para tal, precisaríamos de uma solução para a densidade de energia que decaísse pelo menos mais rápido que $\frac{1}{x^{3}}$, de tal forma que a densidade integrada no espaço, $\int_{0}^{\infty} x^{2} \rho(x) d x$ não fosse para $\infty$ ), o que acaba contribuindo para preencher o universo com uma quantidade infinita de energia. O gráfico a seguir confirma que essa situação ocorre mesmo se utilizarmos o artifício de descontarmos o fundo ${ }^{4}$ na expressão para $\rho(x)$.

\footnotetext{
${ }^{4}$ Onde estamos chamando fundo o valor da densidade de energia $T^{0}{ }_{0}$ para quando $x \rightarrow \infty$.
} 

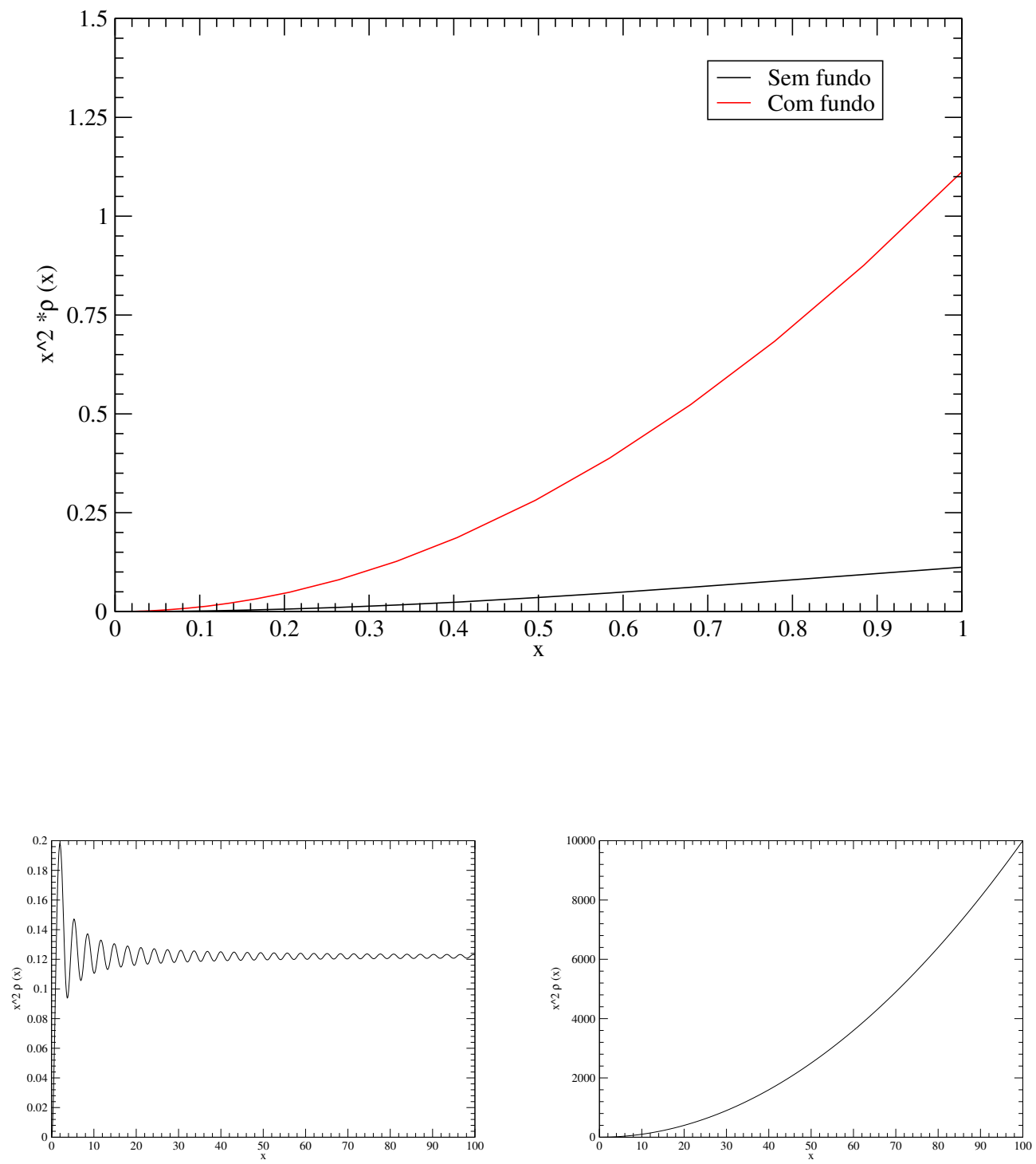

Figura 5.4: Gráficos para $x^{2} \rho(x)$ para uma condição inicial qualquer representativa do comportamento da função $\left(\sigma(0)=0.50, \sigma^{\prime}(0)=0\right)$. Os gráficos mostram regiões distintas do espaço (próxima ou distante da origem) e as diferenças encontradas quando é descontado (esquerda) ou não o fundo. 


\section{Capítulo 6}

\section{Halos de Born-Infeld}

Neste capítulo nos aprofundaremos nas possibilidades de conseguirmos, a partir de um halo formado por um campo de Born-Infeld, resultados que permitam simular características desejáveis para a matéria escura, por exemplo a de contribuir para explicar curvas de rotação de galáxias.

Mostraremos os resultados que obtivemos quando da tentativa de reproduzir cálculos análogos aos já encontrados para uma lagrangeana canônica, conforme visto no Cap. 4. Abandonaremos aqui a primeira abordagem simplista do capítulo anterior. Nosso background nesse momento deixa de ser Minkowski, de tal forma que procuraremos soluções não apenas para o campo mas também para os coeficientes de uma métrica esfericamente simétrica que, por sua vez, será determinada via equações de Einstein e vinculada pela equação de Born-Infeld. Em particular, procuraremos também mostrar uma possível interpretação para o aparecimento da solução de autovalores e autovetores encontrada por [?, ?] e apresentada no Cap. 4, resultado esse que surgiu como subproduto das análises feitas para as equações do campo de Born-Infeld. Baseados nisso, serão analisados vários tipos diferentes de potencial para o campo e de que maneira os mesmos contribuem para alterar as equações de campo, levando a soluções satisfatórias ou não.

\subsection{Simplificação da métrica}

O problema que encontramos consistia basicamente da resolução de um sistema de 4 equações, (três independentes e uma de vínculo), altamente não-lineares e acopladas. A procura então por algum mecanismo ou hipótese que leve à simplificação dos cálculos aparece de forma absolutamente natural. No trabalho que realizamos, a abordagem foi a seguinte: da análise numérica que apresentamos no capítulo anterior, o primeiro detalhe que fomos capazes de perceber dizia respeito a um caráter interessante das soluções. No limite em que o campo e sua derivada espacial tendiam a zero, as equações para a métrica passavam a descr- 
ever na realidade um universo com limite assintótico do tipo Schwarschild-De Sitter e não simplesmente Schwarschild como no caso de [?, ?]. Uma rápida inspeção das equações de Einstein (5.9), (5.10) e (5.11) é capaz de sustentar essa afirmativa. Dessa maneira, estávamos lidando com uma solução a priori fundamentalmente diferente daquela apresentada por [?, ?]. Entretanto, isso não é exatamente surpreendente, na medida que o campo de Born-Infeld aparece com freqüência na literatura como um virtual candidato à energia escura, o que justificaria o aparecimento de um termo com esse caráter, que se comporte como uma espécie de constante cosmológica.

Uma solução como essas, para o caso de uma métrica esfericamente simétrica como em (5.8), corresponderia a uma solução em que $\alpha(x)=-\lambda(x)$ (isso, claro, na região fora do horizonte de eventos). Entretanto, na maior parte do espaço não temos nenhuma consideração óbvia que leve a uma simplificação dessa magnitude para a métrica. Dessa forma, novamente inspirados pelos resultados encontrados por [?, ?], utilizaremos no decorrer de nossa análise uma aproximação na forma $u(x) \approx-v(x)$ em (4.73), buscando a priori alguma forma de correlação entre a solução existente para o caso de uma lagrangeana canônica e para o caso da lagrangeana de Born-Infeld.

\subsection{Liberdade de gauge}

Ainda versando sobre a questão do comportamento assintótico da métrica e do campo, apenas mais algumas ponderações: no limite em que a densidade de energia do campo é constante (de Sitter), podemos reescrever a equação (5.15) para o coeficiente $u(x)$ da métrica como:

$$
u_{0}^{\prime \prime}+2 \frac{u_{0}^{\prime}}{x}=4 \pi G \rho_{0},
$$

Essa equação tem a solução analítica:

$$
u_{0}(x)=u_{o}^{i}+\frac{u_{1}^{i}}{x}+\frac{2 \pi G}{3} \rho_{0} x^{2} .
$$

Para evitar uma descontinuidade na origem, tomamos $u_{1}^{i}$ nulo e ficamos com:

$$
u_{0}(x)=u_{o}^{i}+\frac{2 \pi G}{3} \rho_{0} x^{2} .
$$

sendo $u_{0}^{i}$ o valor inicial de $u_{0}$ na origem.

Porém, de Sitter é um espaço-tempo homogêneo e isotrópico, logo a interpretação de $u_{0}$ como um potencial gravitacional atrativo seria equivocada.

Para a determinação das quantidades físicas do nosso problema, isso nos motiva a definir uma espécie de $u(x)$ efetivo, ou seja, que desconsidere essa contribuição espúria. Optamos por tratar esse problema de uma forma numérica, 
utilizando no cálculo das quantidades físicas (densidade e curvas de rotação) as substituições:

$$
\begin{aligned}
u_{e f f}(x) & =u(x)-u_{0}(x), \\
\rho_{e f f} & =\rho(x)-\rho_{0}(x) .
\end{aligned}
$$

Essas definições separam a parte homogênea do background da parte inomogênea, já que só essa última corresponde aos halos.

\subsection{Ansatz para o campo}

A abordagem que adotamos nesse ponto do trabalho se escora em algumas simplificações importantes, que acabam de certa forma por restringir a classe de soluções possíveis para o problema. Além da já citada simplificação para a métrica, pensamos também em tentar um ansatz para a solução assintótica do campo na forma:

$$
\tilde{\sigma}=\sigma_{0}+\delta \sigma(x)
$$

Essa escolha se justificou a priori pelas várias abordagens numéricas que tentamos, que indicaram fortemente um comportamento como esse para as soluções. Partindo disso, procuramos adquirir uma boa idéia do comportamento geral das soluções encontradas via simulações, visando a construção de um método semianalítico de resolver o problema. Entretanto, essa solução se mostrou ainda mais promissora do que inicialmente imaginado. E a razão para isso é simples, conforme veremos a seguir. Antes disso, porém, façamos uma breve interrupção e olhemos para uma determinada classe de equações diferenciais.

\subsection{Natureza das equações diferenciais}

Olhemos primeiramente para uma equação ordinária simples, do tipo:

$$
y^{\prime \prime}+\frac{2}{x} y^{\prime} \pm k^{2} y=0
$$

onde k é uma constante estritamente real. A solução para a equação com sinal positivo no último termo da esquerda tem a forma:

$$
y_{+}(x)=C_{1} \frac{e^{-i k x}}{x}+C_{2} \frac{e^{i k x}}{x}
$$

Já a solução para a equação com sinal negativo: 


$$
y_{-}(x)=D_{1} \frac{e^{-\sqrt{k^{2}} x}}{x}+D_{2} \frac{e^{\sqrt{k^{2}} x}}{x}
$$

Da análise dessas soluções, fica transparente a existência de duas classes distintas de comportamento para a equação. Temos uma solução com caráter oscilatório (caso do sinal positivo, solução com exponenciais complexas) e outra com caráter exponencial (caso do sinal negativo, exponenciais reais).

A importância disso vai se mostrar evidente no decorrer do capítulo mas, apenas para adiantar, ela reside na seguinte idéia: se conseguirmos manipular o sinal do último termo em (6.6), podemos selecionar o tipo de comportamento para a solução. Desse modo, somos capazes de, de alguma forma, pré-determinar como $y(x)$ se comportará, podendo alterar completamente seu comportamento.

Retornando agora ao Cap. 4, podemos notar que a equação (6.6) tem uma forma funcional bastante semelhante àquela representada por (4.51), o que agora passa a justificar o interesse no estudo de (6.7) e (6.8). A seguir, apresentamos um gráfico que resume de forma ilustrativa as conclusões encontradas no caso da lagrangeana canônica e que serão analisadas para o caso da lagrangeana de Born-Infeld:
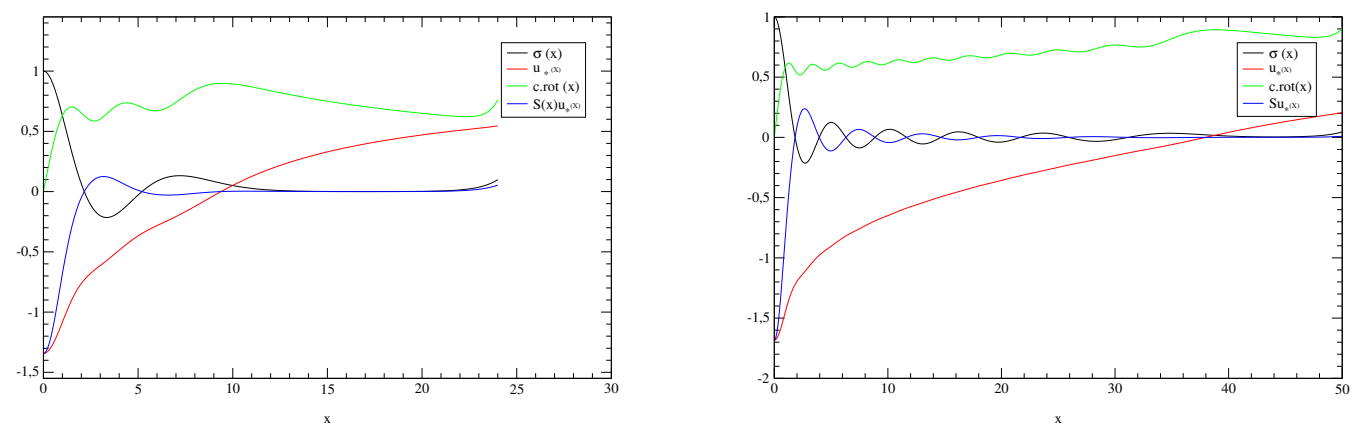

Figura 6.1: Gráficos para a análise do modelo de [?, ?] apresentado no Cap. 4. Nos gráficos, as seguintes condições iniciais foram utilizadas: $\sigma(0)=1, \sigma^{\prime}(0)=0, u_{*}(0)=-1.3437, u_{*}^{\prime}(0)=0$ (caso $n=2)$ e $\sigma(0)=1, \sigma^{\prime}(0)=0, u_{*}(0)=-1.6831$ e $u_{*}^{\prime}(0)=0($ caso $n=10)$.

Olhando para o gráfico (6.1), somos capazes de tecer alguns comentários. O primeiro deles diz respeito ao comportamento suave tanto para o campo quanto para o coeficiente $u_{*}$ da métrica até um $x$ da ordem de $23(n=2)$ e $x$ da ordem de $47(n=10)$ (isso claro, varia de acordo com as condições iniciais). A partir daí, efeitos não-lineares começam a ser dominantes e a solução começa a sair de seu regime de validade. Outro detalhe interessante diz respeito a algo que pode facilmente passar desapercebido na ausência de uma análise mais criteriosa. 
Podemos notar uma suave transição de um regime oscilatório para o campo para um regime com caráter exponencial. Olhando para (6.6) e para o gráfico (6.1), podemos entender o que acontece: no começo da evolução da equação para o campo, $u_{*}(x)$ é negativo, fazendo com que a equação para o campo se encaixe na forma (6.7), $\sigma(x)$ se comportando então como uma solução oscilatória. No decorrer da evolução, entretanto, a situação se inverte, o termo que acompanha $\sigma(x)$ muda de sinal e a solução presente é do tipo exponencial, o que a torna potencialmente interessante, já que um decaimento dessa forma seria suficientemente rápido para não preencher o universo com uma quantidade infinita de energia. O que problema que ainda resta é que temos também a presença de uma solução exponencial crescente, que poderia comprometer o interesse nesse tipo de solução.

Sendo assim, o que procuramos é o seguinte: queremos uma solução que tenha um comportamento oscilatório próximo da origem e um comportamento exponencial à medida que $u_{*}(x)$ for se aproximando de zero. Posteriormente, podemos tentar, mediante uma escolha inteligente das condições iniciais para $u_{*}(x)$, fazer com que a solução exponencial crescente não apareça no resultado final (ou demore o máximo possível pra aparecer), levando a uma solução que decresce exponencialmente. Isso, na prática, é obtido quando escolhemos as condições iniciais que determinam $C_{1}$ e $C_{2}$ em (6.7) de tal modo que, no momento em que ocorrer a transição de uma solução oscilatória para outra exponencial, as condições sejam tais que $D_{2}=0$.

Embora isso possa soar estranho em um primeiro momento, atentamos para o fato de que essa impressão possa ser causada por se tratar de um procedimento numérico. Em uma solução analítica, isso seria bastante natural, sendo equivalente a impormos a regularidade da solução no infinito, ou seja, tomarmos $D_{2}=0$ em (6.8).

$\mathrm{Na}$ realidade, é exatamente esse procedimento que justifica o aparecimento das soluções de autovalores e autovetores apresentada no cap. 4. O espectro discreto encontrado reflete essa característica de que apenas determinadas condições iniciais levam a evoluções para o campo e para a métrica que decaem de maneira satisfatória.

\subsection{Estudo do caso de alguns potenciais do tipo $U=U\left(\Phi, \Phi^{\dagger}\right)$}

Feitas as primeiras considerações, passemos agora ao estudo de casos mais específicos. Com um ansatz do tipo $\tilde{\sigma}=\sigma_{0}+\delta \sigma(x)$ para a parte espacial do campo (6.5), podemos reescrever a equação para o campo de Born-Infeld, (5.18). Se supusermos tanto a correção $(\delta \sigma)$ quanto os parâmetros da métrica pequenos, obtemos a equação aproximada: 


$$
\begin{aligned}
\delta \sigma^{\prime \prime}(x)+\frac{2}{x} \delta \sigma^{\prime}(x)+(1+2 v-2 u)\left(\sigma_{0}+\delta \sigma(x)\right) & \approx \\
{\left[1-(1-2 u)\left(\sigma_{0}^{2}+2 \sigma_{0} \delta \sigma(x)\right)\right]\left[\left.(\ln U)_{, \sigma}\right|_{\sigma_{0}}\right.} & \left.+\left.(\ln U)_{, \sigma \sigma}\right|_{\sigma_{0}} \delta \sigma(x)\right](1+2 v) \\
& +O\left(\delta \sigma^{2}, \delta \sigma^{\prime 2}, u^{\prime} \delta \sigma^{\prime} \ldots\right) .
\end{aligned}
$$

Olhando agora para as várias ordens de aproximação, teremos:

Equação para os termos de ordem zero ${ }^{1}$ em $\delta \sigma$ :

$$
\sigma_{0}=\left.\left(1-\sigma_{0}^{2}\right)(\ln U)_{, \sigma}\right|_{\sigma_{0}}
$$

Equação para os termos de primeira ordem em $\delta \sigma$ :

$$
\begin{aligned}
1+2 v-2 u & =\left[\left.\left(1-{\sigma_{0}}^{2}+2 u \sigma_{0}^{2}\right)(\ln U)_{, \sigma \sigma}\right|_{\sigma_{0}}\right](1+2 v) \\
& +\left[\left.\left(-2 \sigma_{0}+4 u \sigma_{0}\right)(\ln U)_{, \sigma}\right|_{\sigma_{0}}\right](1+2 v) .
\end{aligned}
$$

Nos casos analisados a seguir, a hipótese adicional $u(x) \approx-v(x)$, já citada anteriormente, passará a ser utilizada.

\subsubsection{Potencial do tipo $U\left(\Phi, \Phi^{\dagger}\right)=K \Phi^{p}$}

Da equação (6.10), depois do acréscimo do potencial às equações, obtemos para a solução constante $\sigma_{0}$ :

$$
\sigma_{0}=\left(1-\sigma_{0}^{2}\right) \frac{p}{\sigma_{0}}
$$

que, resolvida, fornece:

$$
\sigma_{0}=\sqrt{\frac{p}{1+p}} .
$$

É importante destacar que a condição imposta por (6.12) vincula fortemente os valores permitidos para p. Para um ansatz como o suposto, só podemos ter um potencial com potências positivas do campo (o que é interessante, já que exclui uma extensa gama de potenciais com potências negativas do campo, bastante comuns em modelos com a lagrangeana de Born-Infeld [?]).

\footnotetext{
${ }^{1}$ Reparar que u e v são tomados zero nessa aproximação.
} 
O gráfico a seguir mostra os valores de $\sigma_{0}$ obtidos via equação (6.12) para um certo intervalo de variação do parâmetro p:

Da equação para os termos de primeira ordem, (6.11), temos:

$$
1-4 u=\left[\left(1-{\sigma_{0}}^{2}+2 u \sigma_{0}^{2}\right)\left(\frac{-p}{\sigma_{0}^{2}}\right)-2 \sigma_{0}(1-2 u)\left(\frac{p}{\sigma_{0}}\right)\right](1-2 u) .
$$

Injetados em (6.9), (6.12) e (6.13), fornecem:

$$
\delta \sigma^{\prime \prime}+\frac{2}{x} \delta \sigma^{\prime}+\left[2\left(1+p+2 u^{2} p-3 u(1+p)\right)\right] \delta \sigma \approx 0 .
$$

Se olharmos essa solução para algum valor de $u(x)$ pequeno $^{2}$, veremos que ela é sempre oscilatória, como pode ser visto do estudo de (6.7). Os gráficos a seguir mostram o comportamento do termo que acompanha $\delta \sigma(x)$ (TADS) em (6.14) para vários valores de $p$ e de $u(x)$. Em particular, apresentamos cortes da Fig (6.2) para alguns valores do parâmetro $p$. É possível observar nesses gráficos que, nesses casos, o campo só teria o comportamento desejado se o efeito da gravitação fosse muito grande $(u \sim O(1))$, o que é indesejado, já que sairíamos do limite newtoniano de validade de nossas aproximações.

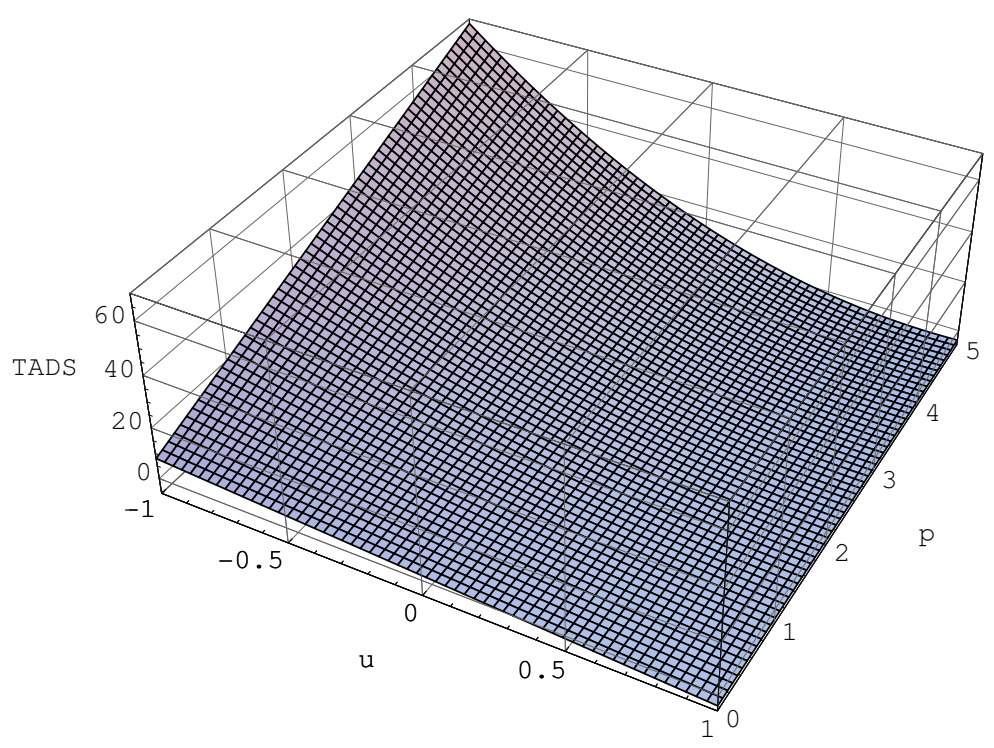

Figura 6.2: Gráfico tridimensional apresentando um trecho do espaço de parâmetros para $u(x)$ e $p$.

\footnotetext{
${ }^{2}$ É evidente que $u(x)$ deve variar com a evolução do campo, mas a idéia aqui é olhar se para algum valor pequeno de $u(x)$ ainda podemos transitar de um tipo de solução para outro.
} 

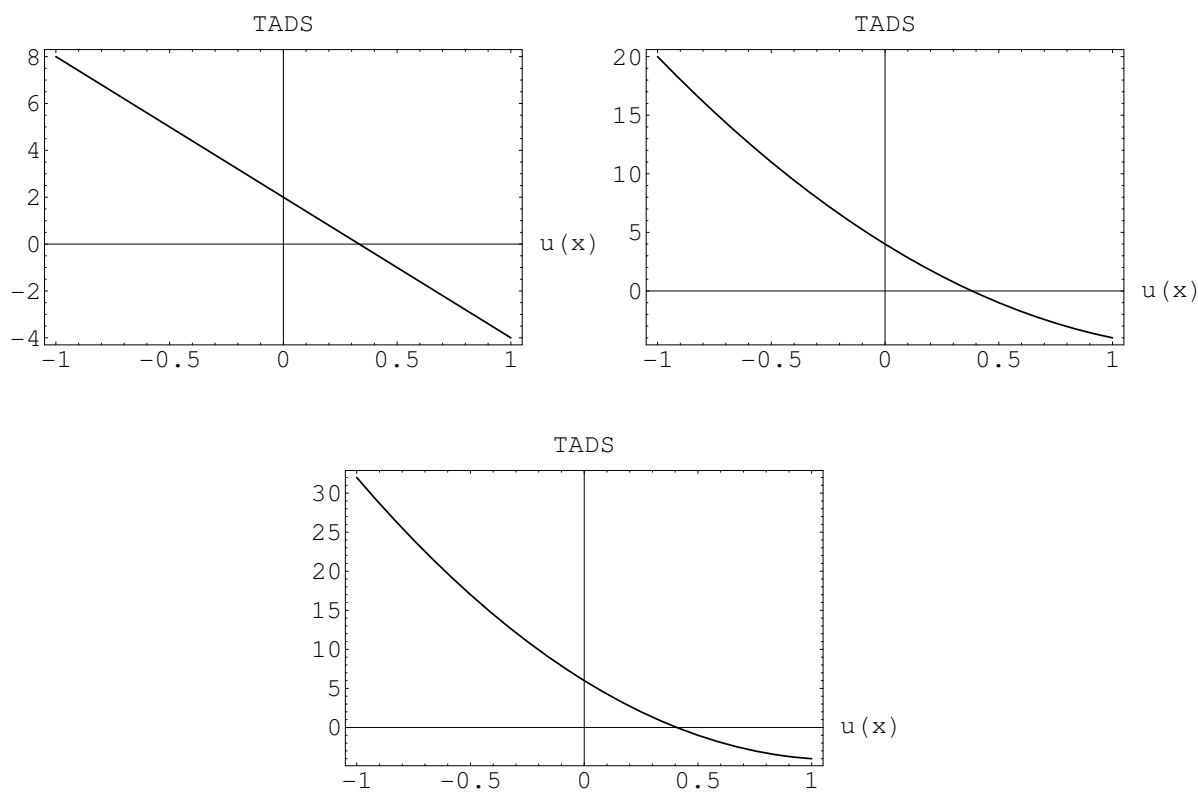

Figura 6.3: Gráficos para alguns valores do parâmetro $\mathrm{p}(p=0,1,2)$ do potencial para o campo. É imediato notar que TADS só se torna negativo (o que permitiria um comportamento do tipo exponencial para o campo) para valores muito grandes e positivos de $\mathrm{u}(x)$.

Também apresentamos a seguir como exemplo os gráficos de $\sigma(x)$ para $p=0$, 1, 2, 3 e 4 (Fig. 6.4). Neles é possível reparar a veracidade da aproximação dada por (6.12), podendo-se notar claramente que as soluções para o campo oscilam em torno de $0.0,0.70,0.81,0.86$ e 0.89 , muito próximo dos valores previstos para $\sigma_{0}$ nesses casos. Além disso, fizemos gráficos para outros valores iniciais dos parâmetros, mas eles não apresentam alterações qualitativas consideráveis e não estão aqui apresentados. 

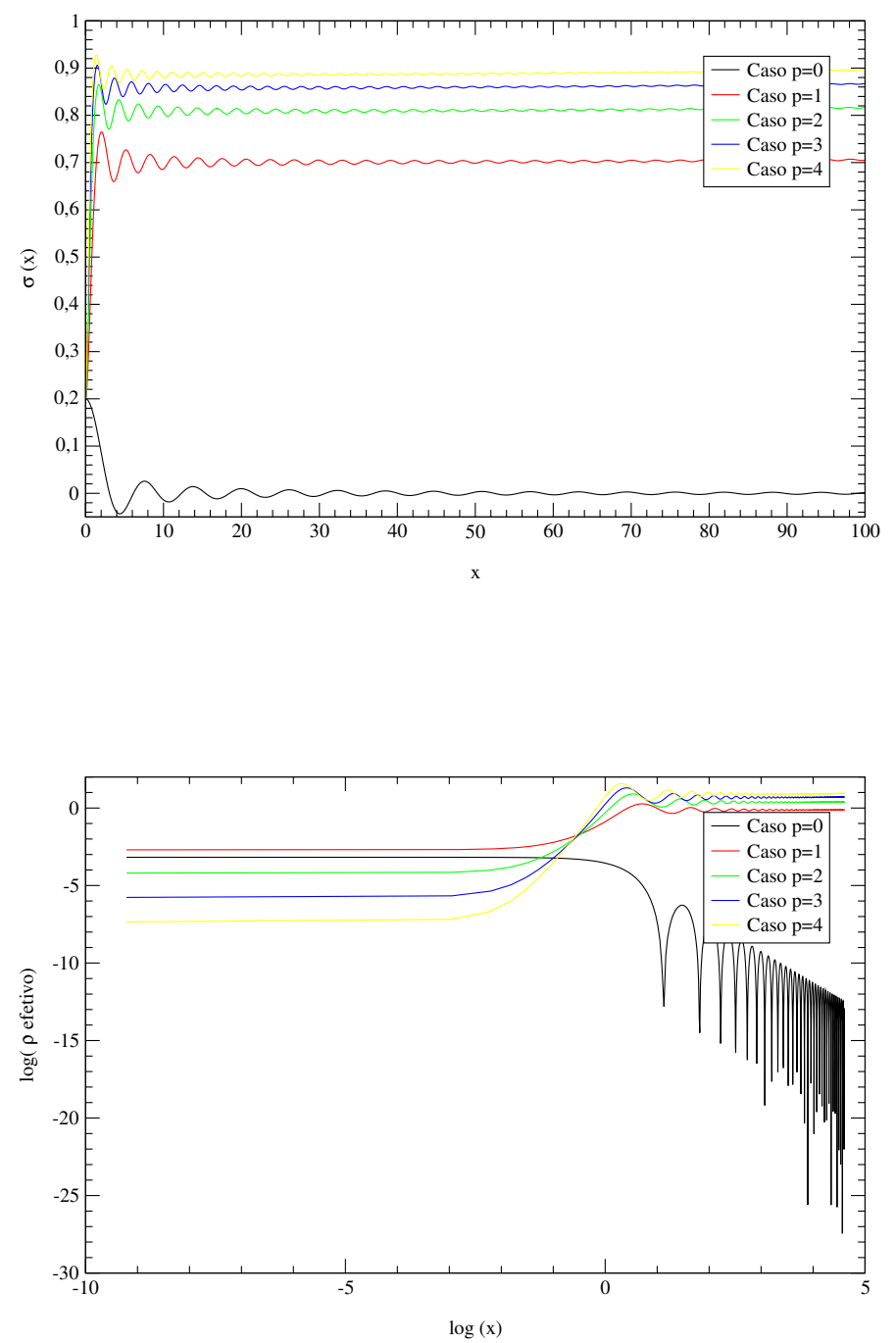

Figura 6.4: Gráficos da solução para o campo e da densidade efetiva para cada um dos casos. Os parâmetros utilizados foram $u(0)=-0.01, u^{\prime}(0)=0, \sigma(0)=0.2, \sigma^{\prime}(0)=0$ e o valor para $\frac{8 \pi G}{\omega^{2}} K=10^{-5}$.

Apresentamos ainda as possíveis curvas de rotação que partículas-teste em espaços-tempo permeados por campos escalares dessa natureza deveriam apresentar. É possível reparar nas curvas que as velocidades próximas ao centro do halo crescem de maneira linear em todos os casos, com uma inclinação para a velocidade circular que aumenta com a potência $p$ do potencial para o campo. 

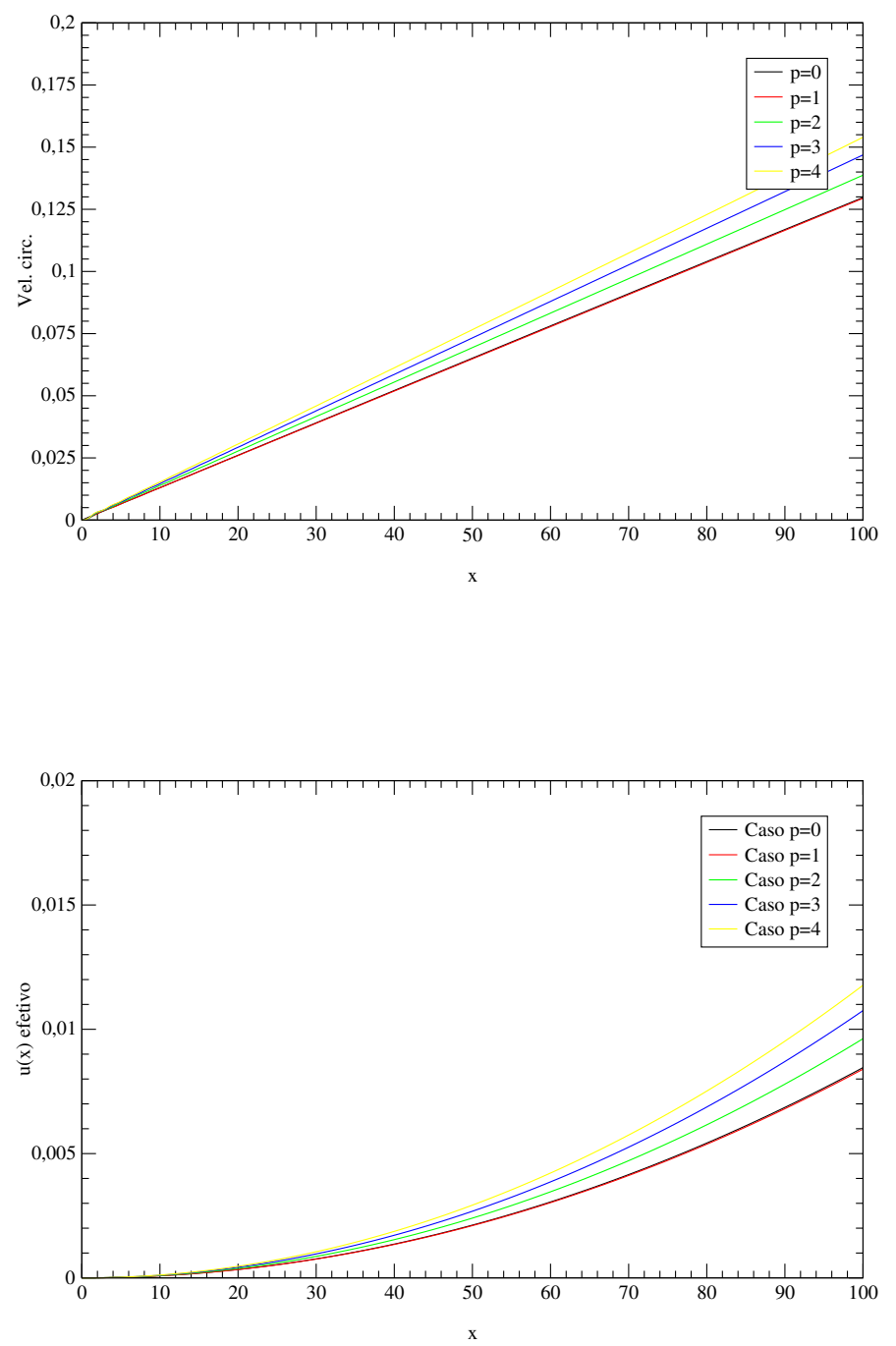

Figura 6.5: Curvas de rotação para as partículas-teste. As curvas foram feitas para uma região onde a aproximação newtoniana para $u(x)$ ainda é válida e os parâmetros utilizados foram os mesmos do gráfico (6.4). O painel inferior apresenta a evolução do parâmetro $u_{e f f}(x)$.

Como a perturbação $\delta \sigma$ sempre oscila nesses casos apresentados, a densidade de energia efetiva do campo perturbado preenche todo o espaço e portanto o modelo com esse tipo de potencial $\left(U\left(\Phi, \Phi^{\dagger}\right)=K \Phi^{p}\right)$ deve ser descartado.

\subsubsection{Potencial do tipo $U\left(\Phi, \Phi^{\dagger}\right)=e^{\beta|\Phi|}$}

Nesse caso, da equação de ordem zero, obtemos: 


$$
\sigma_{0}=\left(1-\sigma_{0}^{2}\right) \beta
$$

que, resolvida, fornece:

$$
\left\{\begin{array}{l}
\sigma_{01}=\frac{-1-\sqrt{1+4 \beta^{2}}}{2 \beta} \\
\sigma_{02}=\frac{-1+\sqrt{1+4 \beta^{2}}}{2 \beta}
\end{array} .\right.
$$

A primeira das soluções, $\sigma_{01}$, tem módulo maior que 1 , devendo portanto ser descartada.

Olhemos então para a segunda das soluções. Essa solução, injetada na eq. (6.9), fornece:

$$
\delta \sigma^{\prime \prime}+\frac{2}{x} \delta \sigma^{\prime}+\left[1-4 u+(1-2 u)^{2}\left(-1+\sqrt{1+4 \beta^{2}}\right)\right] \delta \sigma \approx 0 .
$$

O procedimento a ser realizado é análogo ao anterior. Para $u(x)$ pequeno (aproximação newtoniana), essa solução é também sempre oscilatória, como novamente pode ser visto do estudo de (6.7). A seguir temos o gráfico de TADS para vários valores da constante $\beta$.
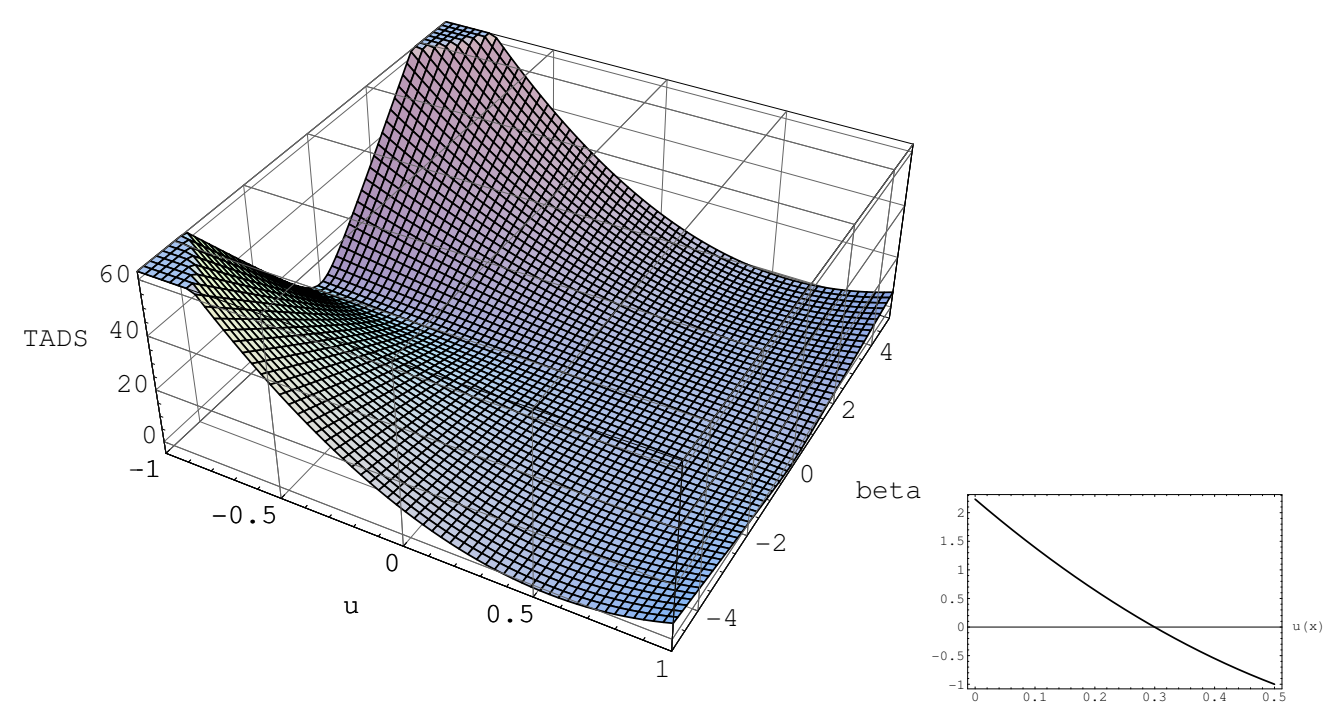

Figura 6.6: Representação tridimensional do espaço do parâmetro $\beta$ e do coeficiente $u(x)$. A direita é apresentado um corte para um caso representativo, quando $\beta=1$. Novamente, TADS só se torna negativo para valores relativamente grandes de $u(x)$. 
Apresentamos também a seguir um gráfico para o campo, curva de rotação para partículas-teste e coeficiente $u_{\text {eff }}(x)$, representativo dos resultados encontrados. O caráter exponencial para o crescimento da solução para o campo fica evidente quando o a métrica começa a ficar grande demais.

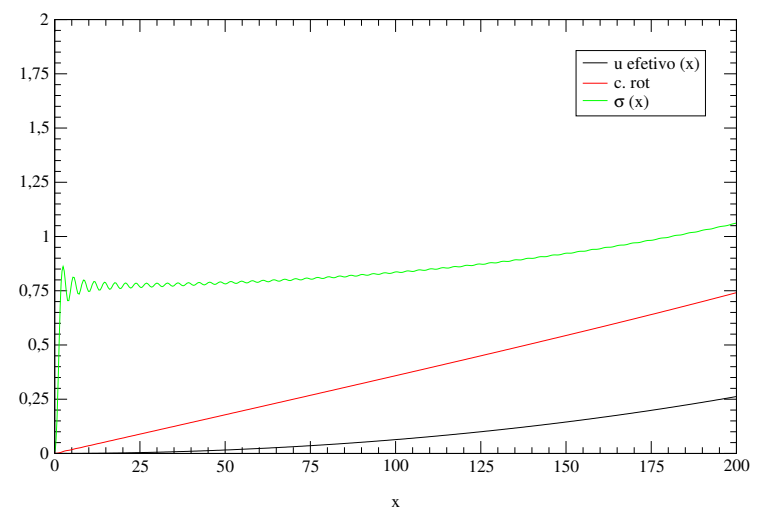

Figura 6.7: Gráfico representativo da evolução dos parâmetros. Nesse caso em particular, $\beta=2$.

\subsubsection{Potencial do tipo $U\left(\Phi, \Phi^{\dagger}\right)=e^{\beta|\Phi|^{2}}$}

A equação de ordem zero fornece:

$$
\sigma_{0}=2\left(1-\sigma_{0}^{2}\right) \beta \sigma_{0}
$$

Cuja solução é dada por:

$$
\left\{\begin{array}{l}
\sigma_{01}=-\frac{\sqrt{-1+2 \beta}}{\sqrt{2 \beta}} \\
\sigma_{02}=\frac{\sqrt{-1+2 \beta}}{\sqrt{2 \beta}}
\end{array} .\right.
$$

A primeira imposição a ser feita nessas duas soluções é que o módulo de $\beta$ seja estritamente maior do que 0.5 , para não termos uma solução imaginária. Além disso, para não termos soluções com módulo maior do que $1, \beta$ não pode ser negativo.

A solução para $\sigma_{01}$ injetada em 6.9 , fornece:

$$
\delta \sigma^{\prime \prime}+\frac{2}{x} \delta \sigma^{\prime}+[-2+4 \beta+4 u(2-5 \beta+u(-3+6 \beta))] \delta \sigma \approx 0,
$$

onde fica fácil notar que a expressão entre colchetes é novamente sempre positiva, para $u(x)$ pequeno e positivo (lembrando sempre que $\beta>0.5$ ). Ou seja, 
novamente um comportamento oscilatório para a solução. Devido às simetrias da expressão para TADS, em que os valores de $\sigma_{0}$ sempre aparecem elevados a potências pares, temos um comportamento idêntico no caso de $\sigma_{02}$. O gráfico a seguir ilustra esse comportamento:

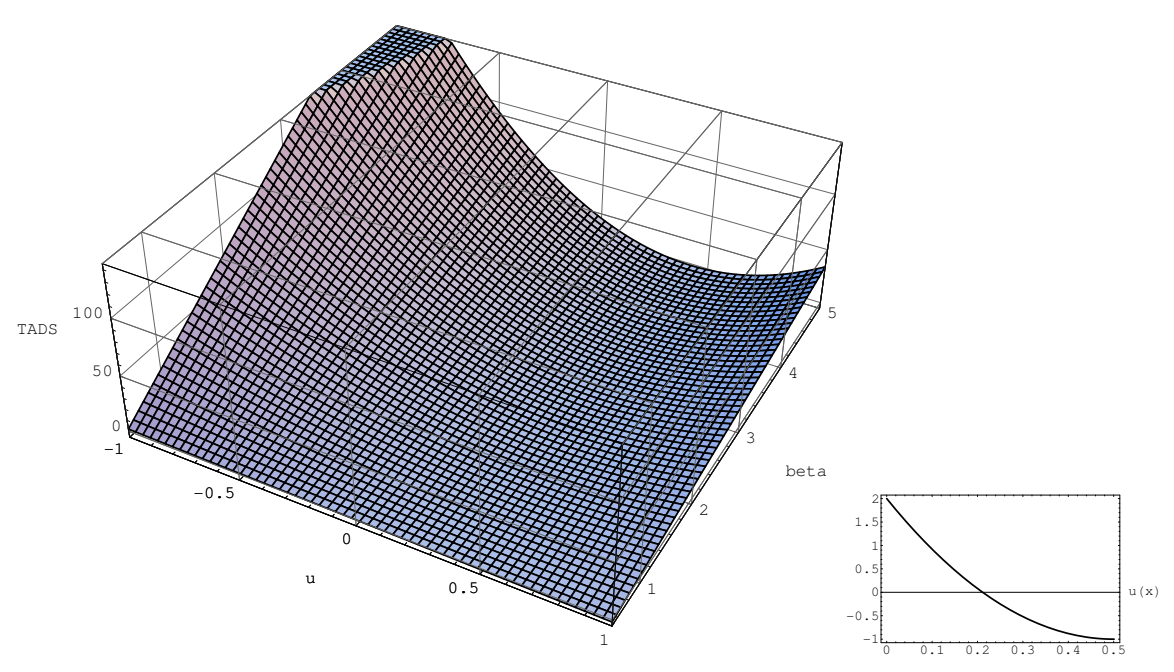

Figura 6.8: Representação dos possíveis valores para o parâmetro $\beta$ e as possíveis implicações para o sinal de TADS. O gráfico a direita é um exemplo representativo das conclusões obtidas, que também requerem um valor alto de $u(x)$ para que se obtenha um TADS negativo.

\subsubsection{Potencial do tipo $U\left(\Phi, \Phi^{\dagger}\right)=e^{\beta|\Phi|^{3}}$}

Esgotadas as possibilidades com potenciais mais convencionais, passamos a buscar os potenciais menos populares, visando encontrar alguma solução que desse conta do comportamento que esperávamos.

Neste caso, a solução constante é fornecida pela equação:

$$
\sigma_{0}=3\left(1-{\sigma_{0}}^{2}\right) \beta \sigma_{0}^{2} \text {. }
$$

Resolvendo (6.18) com auxílio do software Mathematica, obtemos como possíveis soluções para $\sigma_{0}$ : 


$$
\begin{aligned}
& \sigma_{01}=\frac{\left(\frac{2}{3} \beta\right)^{\frac{1}{3}}}{\left(-3+\sqrt{3} \sqrt{3-4 \beta^{2}}\right)^{\frac{1}{3}}}+\frac{\left(-3+\sqrt{3} \sqrt{3-4 \beta^{2}}\right)^{\frac{1}{3}}}{(2 \beta)^{\frac{1}{3}} 3^{\frac{2}{3}}} \\
& \sigma_{02}=\frac{(1+i \sqrt{3}) \beta}{2^{\frac{2}{3}} 3^{\frac{1}{3}}\left[\beta^{\frac{2}{3}}\left(-3+\sqrt{3} \sqrt{3-4 \beta^{2}}\right)^{\frac{1}{3}}\right]}-\frac{(1-i \sqrt{3})\left(-3+\sqrt{3} \sqrt{3-4 \beta^{2}}\right)^{\frac{1}{3}}}{2^{\frac{4}{3}} 3^{\frac{2}{3}} \beta^{\frac{1}{3}}}, \\
& \sigma_{03}=-\frac{(1-i \sqrt{3}) \beta}{2^{\frac{2}{3}} 3^{\frac{1}{3}}\left[\beta^{\frac{2}{3}}\left(-3+\sqrt{3} \sqrt{3-4 \beta^{2}}\right)^{\frac{1}{3}}\right]}-\frac{(1+i \sqrt{3})\left(-3+\sqrt{3} \sqrt{3-4 \beta^{2}}\right)^{\frac{1}{3}}}{2^{\frac{4}{3}} 3^{\frac{2}{3}} \beta^{\frac{1}{3}}}, \\
& \sigma_{04}=0 .
\end{aligned}
$$

Para $|\beta|>\sqrt{3} / 2$, as soluções para o campo são todas reais, conforme podemos constatar nos gráficos a seguir:
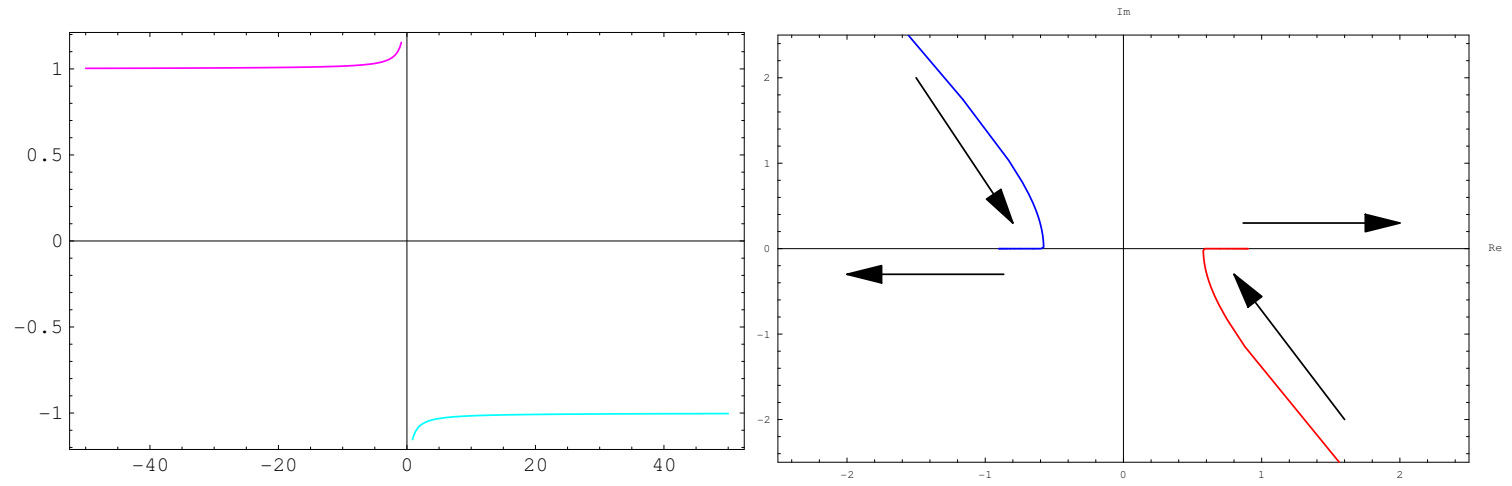

Figura 6.9: O gráfico à esquerda apresenta a solução $\sigma_{01}$ (cujo módulo sempre é $>1$ ) como função de $\beta$. No gráfico à direita, plotaram-se parametricamente as partes real e imaginária das soluções $\sigma_{02}$ e $\sigma_{03}$. As setas indicam o sentido de crescimento de $|\beta|$.

A fim de sintetizar o que foi encontrado, veremos o comportamento para um caso particular, em que $\beta=0.87$, que ilustra bem os resultados além de se situar a solução em uma região onde todas as raízes de (6.19) são reais. Nesse caso, as raízes da equação para $\sigma_{0}$ são: $0,-1.15411,0.545188$ e 0.608926 , conforme pode ser constatado no gráfico seguinte, que apresenta esse resultado: 


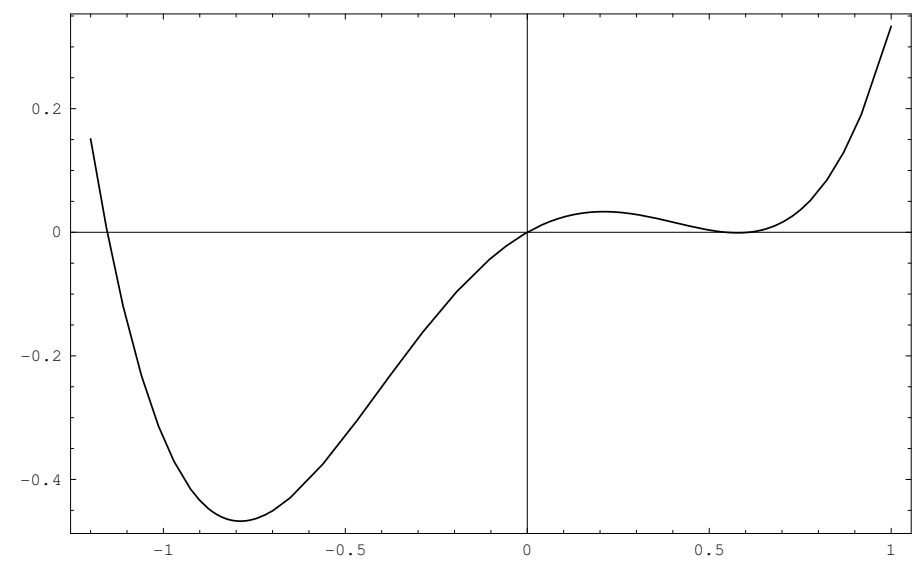

Figura 6.10: Raízes da equação para $\sigma_{0}$.

A solução com $\sigma_{0}=-1.15411$ pode ser descartada por ter um módulo maior do que um. A solução com $\sigma_{0}=0$ resolve trivialmente a equação de primeira ordem para $\delta \sigma$. Olhemos o que acontece com as outras duas. O gráfico abaixo mostra o comportamento de TADS (termo que acompanha $\delta \sigma$ ) para os dois valores restantes de $\sigma_{0}$ (vermelho $\sigma=0.608926$ e azul $\sigma=0.545188$ ).

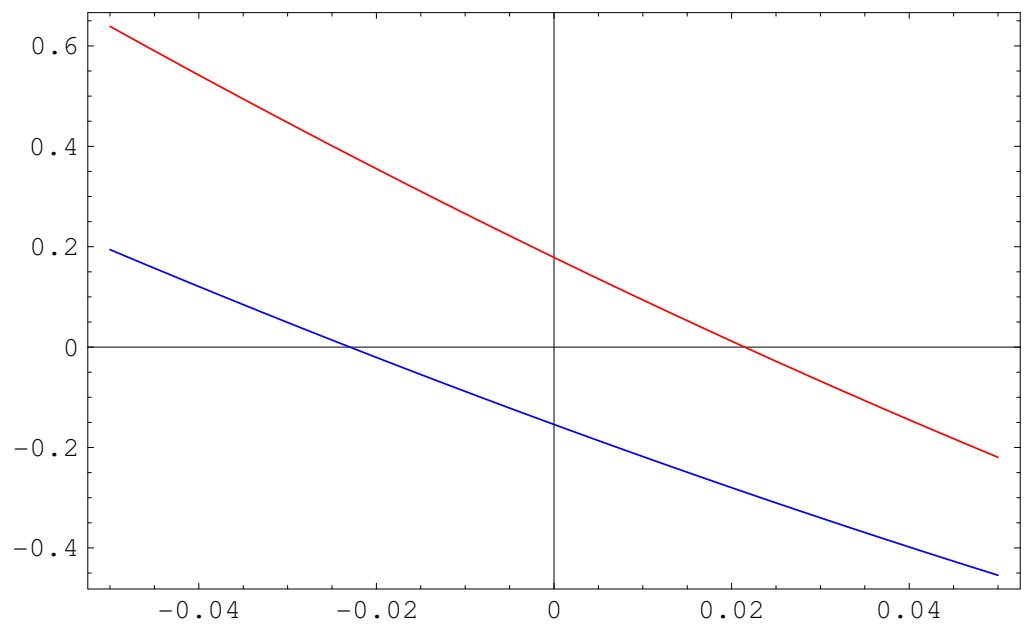

Figura 6.11: TADS como função de $u$.

Da análise do gráfico, fica claro que para $\sigma=0.545188$, existe uma região com $u$ pequeno e ainda negativo que permite que TADS fique negativo, o que poderia dar origem a uma solução com caráter exponencial.

Apresenta-se o gráfico da equação exata para o campo no caso particular em que $u=0$, para verificarmos se a solução exponencial procurada realmente 
aparece para uma dada condição inicial para o campo ou se o sistema procura por nutros nnntes de actahilidade.

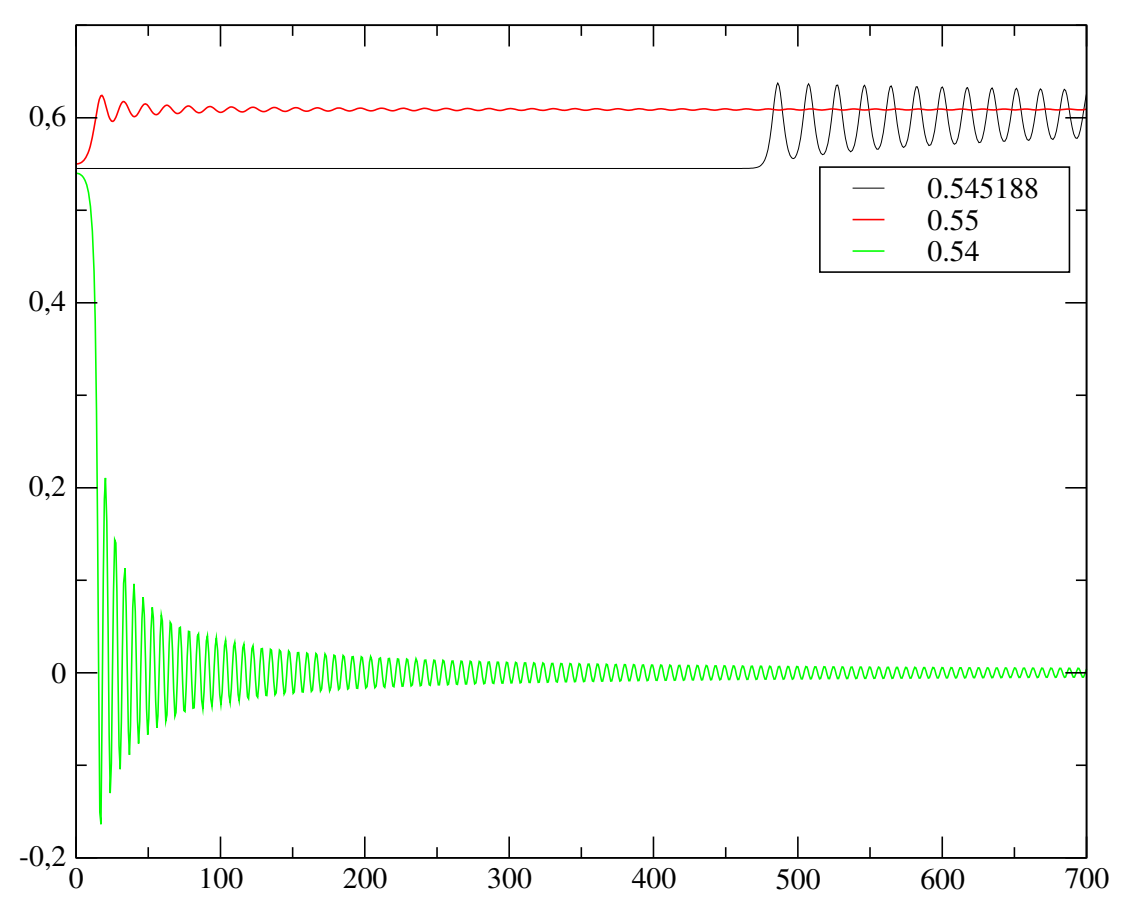

Figura 6.12: $\sigma$ como função de x para várias condições iniciais.

A figura (6.12) deixa bem claro o que está ocorrendo. Para uma condição inicial próxima do ponto 0.545188 o sistema evolui para outras órbitas (ao redor de $\sigma_{0}=0$ ), essas com caráter estritamente oscilatório. Para $\sigma_{0}$ exatamente 0.545188, temos uma solução instável, ela fica constante por um longo intervalo em x, mas com o crescimento das instabilidades ( $\sigma^{\prime}$ começa a ficar muito grande) a solução sai desse ponto e passa também a oscilar, ou seja, o caráter exponencial previsto novamente não surge, ao invés disso $\sigma$ transita para $\mathrm{x}$ grande para uma solução atratora que também apresenta comportamento oscilatório.

\subsection{Considerações finais}

A análise que realizamos para os vários potenciais revela claramente o tipo de comportamento previsto no tópico (6.4) para as soluções do campo. Entretanto, o que foi encontrado no modelo analisado ainda não aparece como uma solução definitiva para os halos, já que o regime de validade das soluções é relativamente pequeno, e serviria desse modo apenas para descrever uma porção mais interna dos halos, o chamado "core", devido ao crescimento rápido do coeficiente $u(x)$ da métrica. O caráter assintótico do espaço-tempo tem presença marcante e sempre 
domina as soluções a partir de certos limites para o raio (que dependem, claro, do potencial adotado). Mas as soluções inspiram idéias, como pode ser visto por exemplo se relaxarmos um pouco essas condições:

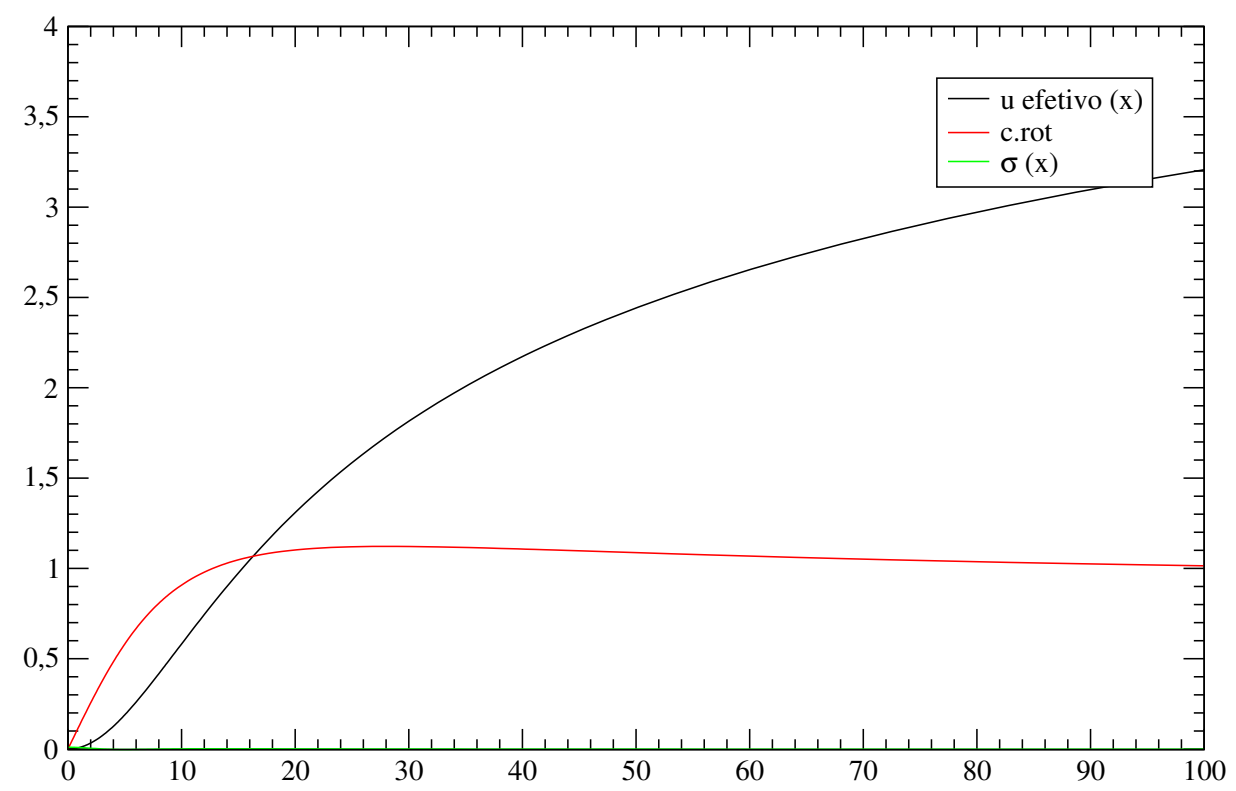

Figura 6.13: Comportamento possível para as curvas de rotação em um caso de potencial do tipo lei de potência, com $\mathrm{p}=0$ (potencial constante).

É evidente que o caráter da curva descrito por (6.13) não pode ser considerado já que foge completamente do limite de validade da aproximação newtoniana, mas soluções como a do gráfico (6.13) apareceram freqüentemente em nossa análise, algumas ligeiramente piores, outras ligeiramente melhores, dependendo fortemente do ajuste dos parâmetros. Nesse caso em particular, é possível inclusive notar a robustez da solução $\sigma_{0}$ para o campo, que permite uma solução suave para a curva de rotação mesmo diante de valores grandes da métrica. 


\section{Capítulo 7}

\section{Conclusões e perspectivas}

O trabalho realizado por nós e apresentado nesse texto constituiu-se basicamente de duas etapas. A primeira delas, que engloba basicamente os três primeiros capítulos, é mais descritiva e apenas busca situar o leitor dentro do paradigma cosmológico atual. A segunda parte, que é embasada pelos capítulos subseqüentes, consiste de uma análise mais criteriosa de algumas características de modelos semi-analíticos para halos de matéria escura, dando especial enfoque a alguns modelos com campos escalares, objetivo final desse texto.

Como pôde ser visto no Cap. 4, fomos capazes de reproduzir e confirmar alguns resultados presentes na literatura versando sobre campos escalares canônicos como fonte de matéria escura, como é por exemplo o caso dos perfis de densidade e das curvas de rotação. Obtivemos ainda de maneira independente o mesmo resultado encontrado por [?, ?] para as condições iniciais propícias a gerarem a mesma solução de autovalores para o campo encontrada por eles, solução essa que permite o aparecimento de modos com densidade de energia finita. Dessa maneira, motivados por essas constatações iniciais, a análise que realizamos para o modelo com a lagrangeana de Born-Infeld foi amplamente baseada e inspirada nessas constatações, sugerindo fortemente as linhas gerais de nosso modelo.

Dentro das pretensões iniciais, pode-se dizer que, de certa forma, os resultados conseguidos para os campos regidos pela lagrangeana de Born-Infeld ficaram aquém do esperado. Embora, como já dito, tenhamos tido sucesso no processo de reprodução de resultados para o caso canônico, nossa tentativa de encontrar resultados análogos para a lagrangeana de Born-Infeld não se concretizou (o que entretanto ainda não sugere que tentativas com potenciais menos convencionais não possam resolver o problema).

Uma consideração que precisa ser feita com respeito ao nosso método diz respeito ao critério utilizado para simplificação das equações de Einstein e do campo de Born-Infeld. Na realidade, não realizamos para o campo de BornInfeld a mesma análise já feita por [?] no caso da lagrangeana canônica, devido à dificuldade encontrada em adaptar essa abordagem para o caso estudado. Desse modo, pondera-se aqui uma possível fragilidade do método. Sendo assim, a aprox- 
imação utilizada $u(x) \approx-v(x)$ pode não ter sido exatamente uma boa solução para o problema. A princípio, acreditávamos que a já conhecida analogia entre as lagrangeanas (canônica e de Born-Infeld) se encarregaria de resolver essa questão e justificaria nossa atitude (o que inclusive motivou definirmos uma lagrangeana com um campo complexo buscando uma comparação direta dos resultados), mas o insucesso em encontrar um potencial com as propriedades que procurávamos (permitir um regime de decaimento exponencial para o campo) inviabilizou esse processo. Dentro desse problema, um possível caminho alternativo que talvez possa ser adotado diz respeito a uma outra maneira de simplificar as equações para o campo. Na analogia das componentes do tensor de energia-momento do campo com as de um fluido, constatamos que lidamos com pressões não anisotrópicas, $p_{r} \neq p_{\theta}$. Esse tipo de comportamento já foi extensamente analisado para o caso das chamadas estrelas de bósons [?] e poderia sugerir maneiras alternativas de lidar com a questão. A escolha de um ansatz para o fator de anisotropia $\left(p_{r}-p_{t}\right)$ poderia ser assim um possível caminho alternativo para simplificar as equações.

Uma outra questão que não foi discutida nesse texto e que talvez possa orientar futuros rumos para o trabalho diz respeito à estabilidade das soluções encontradas. Na realidade, as constatações que fomos capazes de fazer são apenas numéricas, não realizamos nenhum estudo analítico nesse sentido. Para os interessados, as referências [?], que discute a estabilidade de estrelas de bósons e [?], um artigo recente e que talvez não tenha sido aproveitado a contento no decorrer da dissertação, aparecem como boas referências para um estudo nesse sentido.

Também ignorada no trabalho e possível motivação para futuros estudos está a questão acerca da natureza da matéria escura. Nesse caso específico, até que ponto um campo escalar complexo e que portanto descrevesse partículas com carga seria viável?

Agora, apenas para finalizar, como grandes méritos do trabalho, gostaríamos de destacar dois pontos: o primeiro deles diz respeito ao critério descoberto para se descobrir a existência ou não de soluções para o campo que decaem de maneira suficientemente rápida a ponto de gerarem densidades de energia que não gerem contribuições infinitas para a energia total do campo. Até onde fomos capazes de investigar, o critério nos pareceu bom e capaz de justificar a contento os comportamentos encontrados. O segundo ponto, que foi tocado apenas sutilmente durante o decorrer da dissertação, versa sobre a possibilidade até então pouco considerada de que halos de matéria escura possam ser modelados pela lagrangeana de Born-Infeld. Ao final do trabalho, ficamos com a nítida impressão de que esses modelos podem ainda ser muito incrementados e dar boas contribuições nesse sentido. Além de que, devido ao caráter do comportamento assintótico que pode ser constatado para essas soluções, possíveis esquemas de unificação entre matéria escura e energia escura parecem ter vez dentro desses modelos. 


\section{Apêndice A}

\section{Espaço-tempo esfericamente simétrico}

O espaço-tempo plano de Minkowski pode ser descrito em coordenadas esféricas usuais $(r, \theta, \phi)$ com o seguinte elemento de linha:

$$
d s^{2}=d t^{2}-d r^{2}-r^{2}\left(d \theta^{2}+\sin ^{2} \theta d \phi^{2}\right),
$$

onde cada superfície apresentando t e r constante é uma 2-esfera, ou seja uma superfície esférica bidimensional. Nestas superfícies (onde dt $=0$ e dr $=0$ em (A.1)), as distâncias ao longo de curvas são dadas por:

$$
d l^{2}=r^{2}\left(d \theta^{2}+\sin ^{2} \theta d \phi^{2}\right) \equiv r^{2} d \Omega^{2} .
$$

\section{2-esferas em espaço-tempo curvos}

A equação (A.2) permite uma maneira de certa forma mais intuitiva e precisa de definir um espaço-tempo como esfericamente simétrico. De um modo geral, isso poderá ser dito quando todos os pontos do espaço-tempo estiverem sobre uma 2-esfera, ou seja, tiverem um elemento de linha do tipo:

$$
d l^{2}=f\left(r^{\prime}, t\right)\left(d \theta^{2}+\sin ^{2} \theta d \phi^{2}\right),
$$

com $f\left(r^{\prime}, t\right)$ uma função das coordenadas $r^{\prime}$ e $t$. Desta forma, a área de cada uma dessas esferas será dada por $4 \pi f\left(r^{\prime}, t\right)$. Isto nos convida a definir uma coordenada radial $\mathrm{r}$ tal que $f\left(r^{\prime}, t\right) \equiv r^{2}$, o que, por sua vez representa uma mudança de coordenadas $\left(r^{\prime}, t\right) \rightarrow(r, t)$. Esta mudança terá a importante implicação que qualquer superfície com $\mathrm{r}=$ cte e $\mathrm{t}=$ cte será uma 2-esfera de área $4 \pi r^{2}$.

Podemos continuar a análise e olhar para o que deve acontecer para 2-esferas infinitesimalmente próximas (uma em $\mathrm{r}$ e a outra em $\mathrm{r}+\mathrm{dr}$ ) em um 3-espaço com $\mathrm{t}=$ constante. Vejamos que tipo de informação isto pode trazer para a métrica. Nesta situação, o vínculo que pode ser imposto é o seguinte: uma linha com 
$\phi=$ cte, $\theta=$ cte deve necessariamente ser ortogonal às 2-esferas. Ora, por definição esta linha tem simplesmente a direção $\hat{e}_{r}$ (um versor na direção r). Como $\hat{e}_{\theta}$ (versor na direção $\theta$ ) e $\hat{e}_{\phi}$ (versor na direção $\phi$ ) repousam sobre a superfície das 2-esferas, devemos ter:

$$
\hat{e}_{\phi} \cdot \hat{e}_{r}=0 ; \hat{e}_{\phi} \cdot \hat{e}_{r}=0
$$

ou seja, $g_{r \phi}=g_{r \theta}=0$.

Este mesmo argumento pode se estender às outras fatias do espaço, já que por hipótese todo o espaço-tempo é esfericamente simétrico. Isto se traduz na afirmação que uma linha $\operatorname{com} \theta=c t e, \phi=c t e, r=c t e$ também deve ser ortogonal às 2-esferas (do contrário haveria uma direção privilegiada no espaço), o que acarreta:

$$
\hat{e}_{\phi} \cdot \hat{e}_{t}=0 ; \hat{e}_{\phi} \cdot \hat{e}_{t}=0
$$

implicando $g_{t \theta}=g_{t \phi}=0$.

As restrições impostas até agora sugerem então que o elemento de linha para um espaço-tempo esfericamente simétrico:

$$
d s^{2}=+g_{00} d t^{2}-2 g_{0 r} d r d t-g_{r r} d r^{2}-r^{2} d \Omega^{2}
$$

\section{Espaço-tempo estático esfericamente simétrico}

Um espaço-tempo é dito estático quando pudermos nele encontrar uma coordenada temporal t que obedeça as seguintes propriedades: (i) Todos os componentes da métrica são independentes desse t; (ii) a geometria do mesmo não se altera por uma transformação de reversão temporal, ou seja que leva $t \rightarrow-t$. Um exemplo que costuma ajudar a dar uma certa idéia do que isso representa consiste em se imaginar uma esfera girando. Uma reversão temporal (o equivalente a passar o filme de trás pra frente") certamente mudará o sentido da rotação ${ }^{1}$.

A condição (ii) tem uma implicação bastante forte, que limita sobremaneira a métrica que pode descrever um espaço-tempo desta natureza. Ela obriga que uma transformação de coordenadas do tipo: $(t, r, \theta, \phi) \rightarrow(-t, r, \theta, \phi)$ tenha a seguinte matriz de transformação $(\Lambda)$ : (onde barra $(-)$ denota as coordenadas no sistema transformado)

$$
\Lambda_{0}^{\overline{0}_{0}}=-1, \Lambda_{j}^{i}=\delta^{i}{ }_{j}
$$

Isso por sua vez nos permite escrever:

$$
g_{\overline{00}}=\left(\Lambda^{0} \overline{0}\right)^{2} g_{00}=g_{00},
$$

\footnotetext{
${ }^{1}$ É conveniente salientar que um espaço-tempo que seja condizente com (i) mas não com (ii) é dito estacionário.
} 


$$
\begin{gathered}
g_{\overline{0 r}}=\left(\Lambda^{0}{ }_{0} \Lambda_{\bar{r}}^{r}\right) g_{0 r}=-g_{0 r}, \\
g_{\overline{r r}}=\left(\Lambda_{\bar{r}}^{r}\right)^{2} g_{r r}=g_{r r} .
\end{gathered}
$$

Entretanto, como a geometria não deve se alterar da passagem de um sistema de coordenadas para outro, devemos ter $g_{\overline{\alpha \beta}}=g_{\alpha \beta}$, o que por sua vez obriga a identidade $g_{0 r} \equiv 0$. Podemos então escrever genericamente, para um espaçotempo estático esfericamente simétrico a seguinte métrica:

$$
d s^{2}=e^{2 \Phi(r)} d t^{2}-e^{2 \Lambda(r)} d r^{2}-r^{2}\left(d \theta^{2}+\sin ^{2} \theta d \phi^{2}\right) .
$$




\section{Apêndice B}

\section{Universo de Einstein-de Sitter}

Das equações de Friedman, temos que:

$$
\frac{d^{2} a}{d t^{2}}=-\frac{4 \pi}{3} G(\rho(t)+3 p)
$$

e também:

$$
\frac{1}{2}\left(\frac{d a(t)}{d t}\right)^{2}=-\frac{4 \pi}{3} G \rho a^{2}=-\frac{K}{2} .
$$

Tomando a derivada temporal de (B.2), obtemos:

$$
\dot{a} \frac{d^{2} a}{d t^{2}}-\frac{4 \pi}{3} G\left[\left(\frac{d a}{d t}\right) 2 a \rho-\frac{d \rho}{d t} a^{2}\right]=0 .
$$

Por outro lado, multiplicando a equação (B.1) por $-\dot{a}$ e somando com (B.3), obtemos:

$$
-\frac{4 \pi}{3} G\left[\left(\frac{d a}{d t}\right) 2 a \rho-\frac{d \rho}{d t} a^{2}\right]=-\frac{4 \pi}{3} G(\rho(t)+3 p) .
$$

Um pouco de álgebra nos conduz à:

$$
\dot{\rho}=-3 \frac{\dot{a}}{a}(\rho+p) .
$$

Em termos de $\dot{H}=\frac{\dot{a}}{a}$, temos:

$$
\frac{d H}{d t}+H^{2}=-\frac{4 \pi}{3} G \rho(t)
$$

e também:

$$
\dot{\rho}=-3 H(\rho+p) \text {. }
$$


Entretanto, no nosso caso de interesse, por hipótese, estaremos lidando com uma matéria com uma pressão bastante pequena, deprezível quando comparada com a densidade de energia da mesma, de modo que, nesta aproximação $(p<<\rho)$, ficamos com:

$$
\dot{\rho}=-3 H \rho .
$$

Integrando (B.8), obtemos:

$$
\rho=C a^{-3}
$$

onde C é uma constante de integração.

Por outro lado, no modelo de Einstein-De Sitter, temos, por hipótese, $\Omega=1$, ou seja $K=0$, de tal modo que ficamos com:

$$
\left(\frac{d a(t)}{d t}\right)^{2}=\frac{8 \pi}{3} G \rho .
$$

De (B.9) e (B.10), temos:

$$
\begin{gathered}
\left(\frac{d a(t)}{d t}\right)^{2}=\frac{8 \pi G}{3} \frac{C}{a} \\
\sqrt{3} a^{\frac{1}{2}} d a=(8 \pi G C)^{\frac{1}{2}} d t .
\end{gathered}
$$

Integrando esta última expressão, temos:

$$
\frac{2}{3} a^{\frac{3}{2}}=\left(\frac{8 \pi G C}{3}\right)^{\frac{1}{2}} t .
$$

Um pouco de álgebra nos conduz à:

$$
a=(6 \pi G C)^{\frac{1}{3}} t^{\frac{2}{3}}
$$

Utilizando agora (B.10) e (B.12), finalmente obtemos:

$$
\rho(t)=\frac{1}{(6 \pi G)^{\frac{1}{2}} t^{2}} .
$$




\section{Apêndice C}

\section{Abordagem via teoria linear de perturbações}

A dinâmica dos fluidos é governada pelas equações seguintes ${ }^{1}$ :

$$
\begin{gathered}
\text { Equação da continuidade : } \frac{\partial \rho}{\partial t}+\nabla \cdot(\rho \mathbf{v})=0 . \\
\text { Equação de Poisson: } \nabla^{2} \Psi=4 \pi G \rho .
\end{gathered}
$$

$$
\text { Equação de Euler: } \frac{\partial \mathbf{v}}{\partial t}+(\mathbf{v} \cdot \nabla) \mathbf{v}=-\frac{1}{\rho} \nabla \mathbf{p}-\nabla \Psi
$$

onde os parâmetros têm as definições usuais.

Em situações cosmológicas, é útil trabalhar com quantidades comóveis:

$$
\begin{gathered}
\mathbf{x} \equiv \frac{\mathbf{r}}{a(t)}, \\
\mathbf{v} \equiv a(t) \mathbf{x} \\
\delta(\mathbf{x}, t) \equiv \frac{\rho(\mathbf{x}, t)}{\bar{\rho}(t)}-1, \\
\phi(\mathbf{x}, t) \equiv \Psi(\mathbf{x}, t)+\frac{1}{2} a(t) \ddot{a}(t) \mathbf{x}^{2},
\end{gathered}
$$

fazendo com que (C.1), (C.2), (C.3) se reduzam a :

$$
\begin{gathered}
\frac{\partial \delta}{\partial t}+\frac{1}{a}[(1+\delta) \mathbf{v}]=0 . \\
\nabla^{2} \phi=4 \pi G \bar{\rho} \delta a^{2} .
\end{gathered}
$$

\footnotetext{
${ }^{1} \hat{E}$ suposto trabalharmos em escalas onde a teoria newtoniana pode ser aplicada.
} 


$$
\frac{\partial \mathbf{v}}{\partial t}+(\mathbf{v} \cdot \nabla) \mathbf{v}+\frac{\dot{a}}{a} \mathbf{v}=-\frac{1}{\rho a} \nabla p-\frac{1}{a} \nabla \phi
$$

Explorando as chamadas flutuações lineares, onde a amplitude é pequena o bastante $(\delta \ll 1)$, podemos escrever (C.8), (C.9), (C.10), obtendo, para os termos de primeira ordem:

$$
\begin{gathered}
\frac{\partial \delta}{\partial t}+\frac{1}{a} \nabla \mathbf{v}=0, \\
\nabla^{2} \phi=4 \pi G \bar{\rho} \delta a^{2}, \\
\frac{\partial \mathbf{v}}{\partial t}+\frac{\dot{a}}{a} \mathbf{v}=-\frac{c_{s}}{a} \nabla \delta-\frac{1}{a} \nabla \phi,
\end{gathered}
$$

$\operatorname{com} c_{s}^{2} \equiv \frac{\partial p}{\partial \rho}$ a chamada velocidade do som no fluido.

Eliminando-se $\mathbf{v}$ e $\phi$ nas equações acima, é possível obter a equação de evolução para as flutuações de densidade na teoria linear:

$$
\ddot{\delta}+\frac{2 \dot{a}}{a} \dot{\delta}-\left(\frac{c_{s}}{a} \nabla^{2} \delta+4 \pi G \bar{\rho} \delta\right)=0,
$$

cuja solução pode ser encontrada via decomposição em modos de Fourier:

$$
\delta_{k}(t)=\int \delta(\mathbf{x}, t) e^{-i \mathbf{k} \cdot \mathbf{x}} d^{3} \mathbf{x} .
$$

Injetando (C.15) em (C.14), ficamos com:

$$
\ddot{\delta_{k}}+\frac{2 \dot{a}}{a} \dot{\delta}_{k}+\left(\frac{c_{s}{ }^{2} k^{2}}{a^{2}}-4 \pi G \bar{\rho}\right) \delta_{k}=0,
$$

que é a equação para a evolução temporal das flutuaçõs de densidade. Conforme pode ser visto de (C.16), $\delta_{k}$ pode apresentar um modo crescente se tiver um comprimento de onda $\lambda$ maior que um valor crítico $\lambda_{J}$, conhecido pelo nome de comprimento de Jeans:

$$
\lambda=\frac{2 \pi a}{k}>\lambda_{J} \equiv c_{s} \sqrt{\frac{\pi}{G \bar{\rho}}} .
$$

A equação (C.17) indica assim que perturbações com um comprimento de onda menor que o de Jeans oscilam, porque existe um contrabalanço entre a força gravitacional e a pressão.

A equação (C.16) para ser resolvida precisa de uma equação de estado, que estabeleça uma relação entre a pressão e a densidade do fluido. Entretanto, para uma situação de domínio da matéria, em que a pressão pode ser desprezada, ela se simplifica e podemos escrever: 


$$
\ddot{\delta}+\frac{2 \dot{a}}{a} \dot{\delta}-4 \pi G \bar{\rho} \delta_{k}=0 .
$$

A equação (C.18) apresenta duas soluções independentes, um modo crescente denotado por $D_{1}$ e um decrescente $D_{2}$, sendo a solução geral descrita por:

$$
\delta_{k}(t)=C_{1}(\mathbf{k}) D_{1}(t)+C_{2}(\mathbf{k}) D_{2}(t),
$$

onde $C_{1}(\mathbf{k})$ e $C_{2}(\mathbf{k})$ são funções arbitrárias que não dependem do tempo. É sabido que $D_{1}$ e $D_{2}$ obedecem as seguintes relações [?]:

$$
\begin{gathered}
D_{1}(a)=H \int_{0}^{a} \frac{d a^{\prime}}{\left(a^{\prime} H\right)^{3}}, \\
D_{2}=H,
\end{gathered}
$$

em termos do parâmetro de Hubble H. Para um universo em que $a(t) \propto t^{\frac{2}{3}}$, é imediato mostrar de (C.19) que o modo puramente crescente para $\delta_{k}$ também evolui com $t^{\frac{2}{3}}$, assim como no caso não-linear. 


\section{Apêndice D}

\section{Tabela com as conexões}

Para uma métrica esfericamente simétrica, com elemento de linha $d s^{2}$ na forma:

$$
d s^{2}=e^{2 \alpha(\rho)} d t^{2}-e^{2 \lambda(\rho)} d \rho^{2}-\rho^{2} d \Omega^{2},
$$

teremos para as conexões (símbolos de Cristoffel) não-nulas:

\begin{tabular}{|c|c|}
\hline$\Gamma_{11}^{1}=\lambda^{\prime}$ & $\Gamma_{22}^{1}=-\rho e^{-2 \lambda}$ \\
\hline \hline$\Gamma_{12}^{2}=\Gamma_{21}^{2}=\frac{1}{\rho}$ & $\Gamma^{3}{ }_{23}=\Gamma^{3}{ }_{32}=\cot \theta$ \\
\hline$\Gamma^{0}{ }_{10}=\Gamma_{01}^{0}=\alpha^{\prime}$ & $\Gamma^{\circ}{ }_{10}=\Gamma_{01}^{\circ}=\alpha^{\prime}$ \\
\hline$\Gamma^{3}{ }_{13}=\Gamma_{31}^{3}=\frac{1}{\rho}$ & $\Gamma_{00}^{1}=e^{\alpha-\lambda} \alpha^{\prime}$ \\
\hline$\Gamma_{33}^{2}=-\sin \theta \cos \theta$ & $\Gamma_{33}^{1}=-\rho \sin ^{2} \theta e^{-2 \lambda}$ \\
\hline
\end{tabular}

Já para uma métrica esfericamente simétrica na forma isotrópica:

$$
d s^{2}=e^{2 u(r)} d t^{2}-e^{2 v(r)}\left(d r^{2}+r^{2} d \theta+r^{2} \operatorname{sen}^{2} \theta d \phi^{2}\right),
$$

temos:

\begin{tabular}{|c|c|}
\hline$\Gamma_{11}^{1}=v^{\prime}$ & $\Gamma_{22}^{1}=-\left(r+r^{2} v^{\prime}\right)$ \\
\hline \hline$\Gamma_{12}^{2}=\Gamma_{21}^{2}=\frac{1}{r}+v^{\prime}$ & $\Gamma^{3}{ }_{23}=\Gamma^{3}{ }_{32}=\cot \theta$ \\
\hline$\Gamma_{10}^{0}=\Gamma_{01}^{0}=u^{\prime}$ & $\Gamma^{\circ}{ }_{10}=\Gamma_{01}^{\circ}=u^{\prime}$ \\
\hline$\Gamma_{13}^{3}=\Gamma_{31}^{3}=\frac{1}{r}+v^{\prime}$ & $\Gamma_{00}^{1}=e^{u-v} u^{\prime}$ \\
\hline$\Gamma_{33}^{2}=-\sin \theta \cos \theta$ & $\Gamma_{33}^{1}=-\left(r+r^{2} v^{\prime}\right) \sin ^{2} \theta$ \\
\hline
\end{tabular}




\section{Apêndice E}

\section{Roteiro para continuação do trabalho}

Durante o período compreendido entre a entrega da dissertação e a sua apresentação diante da banca examinadora, continuamos trabalhando com os modelos estudados e fomos capazes de perceber alguns detalhes em nosso trabalho que talvez possam ser melhor explorados em pesquisa futuras. Isso nos motivou a incluir esse tópico na versão final entregue, procurando inspirar e possivelmente melhor encaminhar futuros interessados no assunto.

\section{E.1 Possibilidades com um potencial do tipo ex- ponencial}

Um detalhe interessante que ainda não tínhamos percebido, diz respeito aos resultados obtidos com um potencial do tipo exponencial. Dois novos casos interessantes que haviam passado desapercebidos foram descobertos. Particularmente no caso $V(\Phi)=e^{\beta|\Phi|^{3}}$, um detalhe importante nos saltou aos olhos. Para a situação apresentada no Cap. 6, vimos que as melhores soluções encontradas apresentavam um ramo altamente instável, que depois evoluía para uma solução oscilatória. Entretanto, mesmo essa solução oscilatória poderia apresentar em algum momento um comportamento exponencial, já que as duas soluções apresentam uma região com TADS negativo, como pode ser visto na Fig. (6.11). Os resultados anteriores por nós revistos nessa etapa posterior permanecem inconclusivos e merecem mais investigações mas, nas condições apresentadas, para uma integração até um intervalo bastante maior em $x$ (o que ainda não havia sido feito), o comportamento exponencial realmente começa a aparecer. A solução começa oscilatória em torno de um valor qualquer (dependente de $\beta$ ) depois passa a crescer indiscriminadamente.

A dificuldade encontrada nesse caso reside em encontrar uma combinação de parâmetros que de fato permita que, como no caso da lagrangiana canônica, para 
uma determinada condição inicial específica, seja possível diminuir (anular) os efeitos do termo exponencial crescente.

Outro caso que despertou suspeitas foi o do potencial exponencial do tipo $V(\Phi)=e^{\beta \Phi^{2}}$. Nesse caso, para valores de $\beta$ próximos de 0.5 existe uma região no espaço de parâmetros que não ficava clara na Fig. (6.8) e que poderia sim apresentar o tipo de comportamento que desejávamos, para valores ainda pequenos de $u(x)$ poderíamos ter um $T A D S$ trocando de sinal, passando de um valor positivo para outro negativo, levando a solução de um caráter oscilatório para um exponencial.

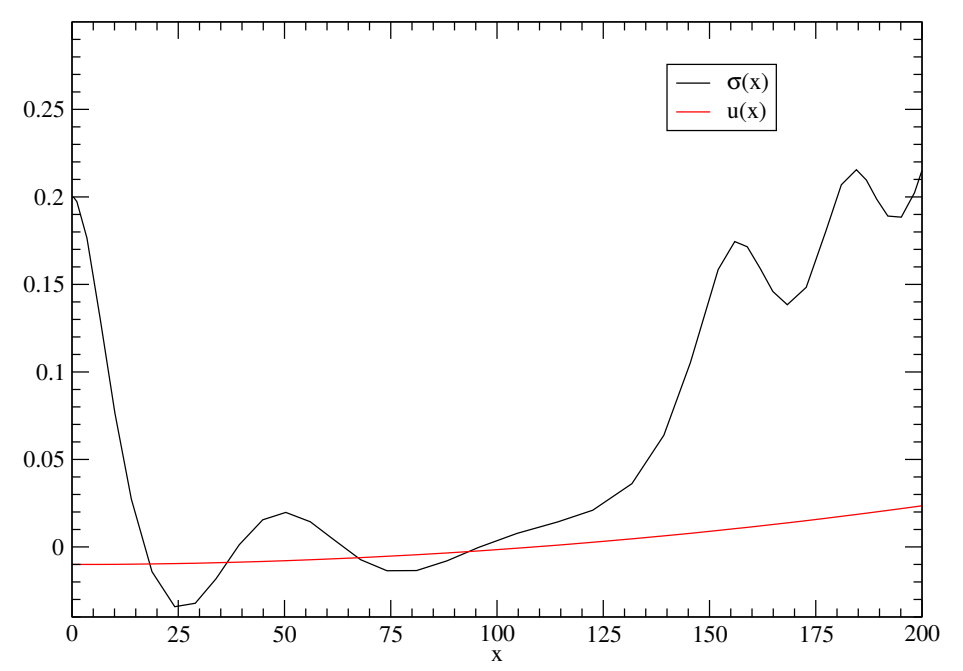

Figura E.1: Caso $V(\Phi)=e^{\beta \Phi^{2}}, \operatorname{com} \beta=0.5, \sigma(0)=0.02, \sigma^{\prime}(0)=0, u(0)=$ $-0.01, u^{\prime}(0)=0$.

Da figura, fica claro o que estamos afirmando. A solução apresenta uma transição de regimes, passa de um comportamento oscilatório para outro exponencial (o que seria desejado) e depois volta a oscilar (o que é difícil controlar). Fizemos experimentações para uma extensa gama de parâmetros, mas esse comportamento qualitativo insiste em aparecer para todos eles. Entretanto, também nesse caso, embora não a tenhamos encontrado, ainda achamos precipitado afirmar que uma condição inicial como a solução dos autovalores do campo escalar canônico (Cap. 4) não possa existir. 


\section{E.2 Como relacionar os modelos com campos es- calares com os dados observacionais?}

Algo que certamente não pôde ser explorado a contento neste texto diz respeito à como fazer a ligação entre o que é efetivamente observado e o modelo teórico baseado em campos escalares. Na realidade, nosso trabalho não permitiu esse tipo de análise, já que não fomos capazes de encontrar halos finitos para o nosso modelo (com a lagrangiana de Born-Infeld). Entretanto, a questão permanece e nossa intenção aqui é esboçar uma idéia baseada na literatura [?, ?, ?] de como isso poderia ser feito.

A situação a ser discutida é a seguinte: imaginemos que o campo escalar seja de fato capaz de gerar um halo de matéria escura. Nesse caso, como encontrar qual a massa do campo que permite que essa configuração se estabeleça? Para o campo escalar canônico, a idéia é se apoiar na hipótese de que a matéria escura deve ser a mesma independente da escala de observação, ou seja, o campo que supostamente seria responsável por seus efeitos deve estar associado a uma determinada massa $m_{\Phi}$. Em termos práticos, isso equivale a afirmar que a matéria escura em galáxias ou aglomerados deve ser a mesma, o que nos fornece uma pista de como proceder. Por um lado, temos fortes vínculos para a massa do campo advindos da física de partículas (vide breve discussão ao final do Cap. 3) enquanto, por outro, a teoria deve ser capaz de, por exemplo, fornecer uma explicação plausível para as curvas de rotação de todas as galáxias utilizando um mesmo valor da massa $m_{\Phi}$ (e também de $\omega$, no caso do campo escalar complexo).

Um possível caminho para empreender essa proposta consiste na adição às equações de Einstein de uma contribuição de densidade de energia devido ao disco luminoso das galáxias. É claro que tal procedimento não é tão trivial, já que, para mantermos a simetria esférica da métrica, há a necessidade de supormos um disco que também possua tal propriedade. No caso das galáxias espirais com baixo brilho superficial essa aproximação deve se mostrar relativamente boa, já que a contribuição proporcional do disco em relação à massa total será pequena ${ }^{1}$.

A contribuição mencionada deve transformar as equações (4.104) e (4.105) deixando-as na seguinte forma:

$$
\begin{gathered}
u_{*}^{\prime \prime}+\frac{2}{x} u_{*}^{\prime}=2 S^{2}+R, \\
S^{\prime \prime}+\frac{2}{x} S^{\prime}=2 u_{*} S,
\end{gathered}
$$

onde $R=\frac{4 \pi G}{m^{2} \bar{\sigma}^{2}(0)} \rho$. Se tivermos (modelo fenomenológico) algum ajuste para as velocidades circulares no disco (usualmente como função do raio $v_{d i s c}(r)$ e do raio

\footnotetext{
${ }^{1}$ É claro que em outros casos uma métrica muito mais complicada deve ser utilizada nas equações.
} 
ótico $v_{\text {disc }}\left(r_{\text {opt }}\right)$ ), podemos obter $\rho$ e conseqüentemente $R$ como função de $v_{d i s c}$, já que devemos ter, da equação de Poisson:

$$
\vec{\nabla} \cdot(\vec{\nabla} \Phi)=4 \pi G \rho \rightarrow \frac{v_{d i s c}^{2}}{r^{2}}+\frac{2 v_{d i s c} v_{d i s c}^{\prime}}{r}=4 \pi G \rho .
$$

Para $R$ nulo em (E.1), devemos redescobrir a solução de autovalores já discutida no Cap. 4. Dessa forma, o raciocínio agora é simples: devemos escrever $R$ como função de de $x, x_{o p t}$ e de $v_{d i s c}$. Sendo assim, dados $x_{o p t}$ e $v_{d i s c}$, o comportamento da solução dada por $S$ fica unicamente determinado.

O trabalho de [?] apresenta um abordagem dessa forma para uma expressão da velocidade circular do disco na forma:

$$
v_{d i s c}^{2}(r)=v_{d i s c}^{2}\left(r_{o p t}\right) \frac{1.97\left(\frac{r}{r_{o p t}}\right)^{1.22}}{\left[\left(\frac{r}{r_{o p t}}\right)^{2}+0.78^{2}\right]^{1.43}},
$$

a qual, inserida em (E.1) e (E.2) permite reescrever:

$$
R=\frac{v_{d i s c}^{2}\left(r_{o p t}\right)}{\bar{\sigma}(0)}\left[\frac{1}{x^{2}} f\left(\frac{x}{x_{o p t}}\right)\right]=\frac{\gamma}{\alpha^{2}} f\left(\frac{x}{\alpha}\right)
$$

onde

$$
\alpha \equiv x=m r_{\text {opt }} \sqrt{\bar{\sigma}(0)}
$$

$\mathrm{e}$

$$
\gamma=\frac{v_{d i s c}^{2}\left(r_{o p t}\right)}{\bar{\sigma}(0)}
$$

Com uma análise de $\chi^{2}$, pode-se procurar pelos valores mais prováveis dos parâmetros $\alpha$ e $\gamma$ e a partir daí, para cada galáxia, pode-se ajustar o valor das velocidades circulares através do parâmetro $\bar{\sigma}(0)$. Por fim, o valor da massa do campo escalar fica vinculada por (E.4) e (E.5) :

$$
m^{2}=\frac{\alpha^{2} \gamma}{r_{o p t}^{2} \beta v^{2}\left(r_{o p t}\right)},
$$

onde $\beta=\frac{v_{d i s c}^{2}}{v^{2}\left(r_{\text {opt }}\right)}$. Dessa forma, o modelo para ser considerado bom precisa ajustar um grande número de curvas de rotação com o mesmo valor do parâmetro $m$ e também de $\omega$. O valor encontrado por [?] é de um $m$ da ordem de $10^{-24} \mathrm{eV}$. 